\title{
A SPLITTING METHOD USING DISCONTINUOUS GALERKIN FOR THE TRANSIENT INCOMPRESSIBLE NAVIER-STOKES EQUATIONS
}

\author{
Vivette Girault ${ }^{1}$, Béatrice Rivière ${ }^{2}$ and Mary F. Wheeler ${ }^{3}$
}

\begin{abstract}
In this paper we solve the time-dependent incompressible Navier-Stokes equations by splitting the non-linearity and incompressibility, and using discontinuous or continuous finite element methods in space. We prove optimal error estimates for the velocity and suboptimal estimates for the pressure. We present some numerical experiments.
\end{abstract}

Mathematics Subject Classification. 65M12, 65M15, 65M60.

Received: September 1, 2004.

\section{INTRODUCTION}

The Navier-Stokes equations characterize a variety of flows, which play an important role in many engineering applications. For incompressible flows, the momentum and continuity equations are:

$$
\boldsymbol{u}_{t}-\mu \Delta \boldsymbol{u}+\boldsymbol{u} \cdot \nabla \boldsymbol{u}+\nabla p=\boldsymbol{f}, \quad \nabla \cdot \boldsymbol{u}=0,
$$

where $\boldsymbol{u}$ is the fluid velocity, $p$ the pressure, $\mu>0$ the constant viscosity, and $\boldsymbol{f}$ a given external force. These equations are completed by adequate boundary and initial conditions.

These equations are difficult to solve numerically because on one hand, they are nonlinear and on the other hand, the velocity is coupled with the pressure. In this paper, we study a particular operator splitting technique introduced by Blasco et al. [5] in 1997, for decoupling the convection and pressure terms. It is convenient to describe the general idea of this splitting technique at the semi-discrete time level; given an approximation $\boldsymbol{U}^{j}$ of the velocity $\boldsymbol{u}\left(t^{j}\right)$ at time $t^{j}$ and an approximation $\boldsymbol{f}^{j+1}$ of $\boldsymbol{f}\left(t^{j+1}\right)$, the computation of the discrete velocity and pressure at time $t^{j+1}$ proceeds in two steps:

1) Linearized convection step: solve for an intermediate velocity $\tilde{\boldsymbol{U}}^{j+1}$ satisfying

$$
\frac{1}{\Delta t}\left(\tilde{\boldsymbol{U}}^{j+1}-\boldsymbol{U}^{j}\right)-\mu \Delta \tilde{\boldsymbol{U}}^{j+1}+\boldsymbol{U}^{j} \cdot \nabla \tilde{\boldsymbol{U}}^{j+1}=\boldsymbol{f}^{j+1} .
$$

\footnotetext{
Keywords and phrases. Operator splitting, time-dependent Navier-Stokes, SIPG.

1 Université Pierre et Marie Curie, Paris VI, Laboratoire Jacques-Louis Lions, 4, place Jussieu, 75252 Paris Cedex 05, France. girault@ann.jussieu.fr

2 Department of Mathematics, University of Pittsburgh, 301 Thackeray, Pittsburgh, PA 15260, USA. riviere@math.pitt.edu

3 Center for Subsurface Modeling, Institute for Computational Engineering and Sciences, University of Texas, 201 E. 24th St., Austin TX 78712, USA. mfw@ices.utexas.edu 
2) Incompressibility step: solve for $\boldsymbol{U}^{j+1}$ and $P^{j+1}$ satisfying

$$
\begin{gathered}
\frac{1}{\Delta t}\left(\boldsymbol{U}^{j+1}-\tilde{\boldsymbol{U}}^{j+1}\right)-\mu \Delta\left(\boldsymbol{U}^{j+1}-\tilde{\boldsymbol{U}}^{j+1}\right)+\nabla P^{j+1}=\mathbf{0}, \\
\nabla \cdot \boldsymbol{U}^{j+1}=0 .
\end{gathered}
$$

If the space discretization is well chosen, this splitting technique has the advantages that

1) the first step reduces to a system of scalar equations, that can be solved in parallel;

2) the discrete velocity obtained from the second step is locally conservative; and

3) the boundary condition can be enforced at each step.

There are several strategies for space discretizations that benefit from part or all of these advantages. For instance,

1) both steps can be solved by a symmetric or non-symmetric discontinuous Galerkin method;

2) the second step can be solved by a discontinuous Galerkin method while the first step can be solved by a continuous finite element method in some appropriate region (possibly the entire region) and by a discontinuous Galerkin method in other regions;

3) the domain can be subdivided into regions in which both steps are solved either by a discontinuous or by a nonconforming finite element method.

The idea of decoupling the nonlinearity from the incompressibility condition dates back to the work of Chorin [7] and Temam [27]. This method was known as the projection method and since then, it has been studied and modified by several authors. The reader can find a good historical account in the introduction of [4] by Blasco and Codina. Without being exhaustive, let us quote the work of Yanenko [31] on fractional step methods, the work of Fernandez-Cara and Beltram [11], Rannacher [25], Turek [29], Guermond and Quartapelle [17], Quarteroni et al. [24], Almgren et al. [2], Guermond and Shen [18,19], all on projection methods. We refer to the recent book of Glowinski [15] for a very comprehensive treatment of fractional step methods and projection methods. The splitting technique of [4] studied here can be viewed as a very particular case of fractional step methods in which the time advances by a full time step. On the other hand, it differs from the above-mentioned projection methods because it projects in $H^{1}$ instead of $L^{2}$. Thus it is more complex than the standard projection method, but in contrast it preserves the boundary condition and produces no artificial boundary layer.

To our knowledge, there is very little in the literature on the analysis of discontinuous Galerkin methods for Navier-Stokes equations. The Symmetric Interior Penalty Galerkin (SIPG) method (originally called interior penalty method) and Non-symmetric Interior Penalty Galerkin (NIPG) method were first introduced for elliptic problems by Wheeler [30] and Rivière et al. [26]. The idea of using a non-symmetric form without penalty was introduced by Baumann and Oden [3]. The NIPG and SIPG methods for the steady-state Navier-Stokes equations were first formulated and analyzed in Girault et al. [14]. In Kaya and Rivière [20] both NIPG and SIPG methods coupled with a subgrid eddy viscosity method are applied to the time-dependent NavierStokes problem. The method we propose here employs the SIPG or NIPG methods, i.e. the bilinear form that approximates the viscous term is either symmetric or non-symmetric. Our numerical experiments with both methods in Section 7 give accurate results.

The discontinuous Galerkin methods present several advantages: they are easily used on highly unstructured meshes, they are locally conservative and they lend themselves well to domain decomposition. Furthermore, the approach we propose satisfies a compatibility condition, which is important in air and water quality modeling (see Rem. 1.5).

Moreover, as far as the Navier-Stokes equations are concerned, discontinuous Galerkin methods lend themselves easily to an upwinding of the convection term, as was studied by Lesaint and Raviart [21] in neutron transport.

The coupling of the continuous regions with the discontinuous regions is useful in many applications such as surface flow (see the application to shallow water in [9]), where the cost of using fully discontinuous Galerkin methods can be reduced. However, in the forthcoming analysis, we shall see that we lose optimality if the 
finite elements change when we pass from step 1 to step 2. In this respect, combining a simple continuous finite element method with a discontinuous Galerkin method requires less degrees of freedom but is less attractive than combining an appropriate nonconforming method with a discontinuous Galerkin method. The nonconforming approach, which is locally conservative, appears to be a good compromise between strategies 1 and 2 . It is interesting to note that none of the analysis below requires a quasi-uniform triangulation.

Although this method and most of its analysis apply to 3D, for simplicity, we shall only approximate the Navier-Stokes equations in 2D. The full problem is:

$$
\begin{aligned}
\boldsymbol{u}_{t}-\mu \Delta \boldsymbol{u}+\boldsymbol{u} \cdot \nabla \boldsymbol{u}+\nabla p & =\boldsymbol{f}, \quad \text { in } \quad \Omega \times(0, T), \\
\nabla \cdot \boldsymbol{u} & =0, \quad \text { in } \Omega \times(0, T), \\
\boldsymbol{u} & =\mathbf{0}, \quad \text { on } \partial \Omega \times(0, T), \\
\boldsymbol{u} & =\boldsymbol{u}_{0}, \quad \text { in } \quad \Omega \times\{0\},
\end{aligned}
$$

where $\Omega$ is a domain in $\mathbb{R}^{2}$ with Lipschitz-continuous boundary $\partial \Omega$. As usual, we write formally:

$$
\boldsymbol{u} \cdot \nabla \boldsymbol{v}=\sum_{i=1}^{2} u_{i} \frac{\partial \boldsymbol{v}}{\partial x_{i}} \quad \text { and } \quad \nabla \cdot \boldsymbol{u}=\sum_{i=1}^{2} \frac{\partial u_{i}}{\partial x_{i}}
$$

It is well known that if $\boldsymbol{f} \in L^{2}\left(0, T ; H^{-1}(\Omega)^{2}\right)$ and $\boldsymbol{u}_{0} \in H(\operatorname{div}, \Omega)$, then this problem has a unique solution $\boldsymbol{u} \in L^{2}\left(0, T ; H_{0}^{1}(\Omega)^{2}\right) \cap L^{\infty}\left(0, T ; L^{2}(\Omega)^{2}\right), p \in W^{-1, \infty}\left(0, T ; L_{0}^{2}(\Omega)\right)$ and $\boldsymbol{u}_{t} \in L^{2}\left(0, T ; \boldsymbol{V}^{\prime}\right)$ (see Lions [23], Temam [28], Girault and Raviart [13]). Here, $L^{2}(\Omega)$ is the classical space of square-integrable functions with the innerproduct $(f, g)=\int_{\Omega} f g, L_{0}^{2}(\Omega)$ is the subspace of functions of $L^{2}(\Omega)$ with zero mean value:

$$
L_{0}^{2}(\Omega)=\left\{v \in L^{2}(\Omega): \int_{\Omega} v=0\right\},
$$

and $H^{1}(\Omega)$ denotes the classical Sobolev space:

$$
H^{1}(\Omega)=\left\{v \in L^{2}(\Omega): \nabla v \in\left(L^{2}(\Omega)\right)^{2}\right\} .
$$

By definition, $H_{0}^{1}(\Omega)$ is the closure of $\mathcal{D}(\Omega)$ in $H^{1}(\Omega)$, where $\mathcal{D}(\Omega)$ is the space of infinitely differentiable functions with compact support, $H^{-1}(\Omega)$ is the dual of $H_{0}^{1}(\Omega), \boldsymbol{V}$ is the space of functions of $\left(H_{0}^{1}(\Omega)\right)^{2}$ with zero divergence:

$$
\boldsymbol{V}=\left\{\boldsymbol{v} \in\left(H_{0}^{1}(\Omega)\right)^{2}: \quad \nabla \cdot \boldsymbol{v}=0\right\}
$$

and $\boldsymbol{V}^{\prime}$ is its dual space. It is well known that $H_{0}^{1}(\Omega)$ is characterized as the subspace of functions of $H^{1}(\Omega)$ that vanish on $\partial \Omega$. More generally, we shall use the spaces

$$
W^{1, r}(\Omega)=\left\{v \in L^{r}(\Omega): \nabla v \in\left(L^{r}(\Omega)\right)^{2}\right\},
$$

equipped with the semi-norm

and norm for which it is a Banach space:

$$
|v|_{W^{1, r}(\Omega)}=\left(\int_{\Omega}|\nabla v|^{r}\right)^{1 / r}
$$

$$
\|v\|_{W^{1, r}(\Omega)}=\left(\|v\|_{L^{r}(\Omega)}^{r}+|v|_{W^{1, r}(\Omega)}^{r}\right)^{1 / r} .
$$

These definitions are extended in the usual way to $r=\infty$. We shall also use

$$
H^{2}(\Omega)=\left\{v \in H^{1}(\Omega): \nabla v \in\left(H^{1}(\Omega)\right)^{2}\right\}
$$


with the semi-norm

$$
|v|_{H^{2}(\Omega)}=|\nabla v|_{H^{1}(\Omega)}
$$

and the norm

$$
\|v\|_{H^{2}(\Omega)}=\left(\|v\|_{H^{1}(\Omega)}^{2}+|v|_{H^{2}(\Omega)}^{2}\right)^{1 / 2} .
$$

We refer to Adams [1], Lions and Magenes [22] for these spaces and for extending them to fractional exponents. As usual, for handling time-dependent problems, it is convenient to consider functions defined on a time interval $(a, b)$ with values in a functional space, say $Y$ (see [22]). More precisely, let $\|\cdot\|_{Y}$ denote the norm of $Y$; then for any number $r, 1 \leq r \leq \infty$, we define

$$
L^{r}(a, b ; Y)=\left\{f \text { measurable in }(a, b): \int_{a}^{b}\|f(t)\|_{Y}^{r} \mathrm{~d} t<\infty\right\}
$$

equipped with the norm $\|f\|_{L^{r}(a, b ; Y)}=\left(\int_{a}^{b}\|f(t)\|_{Y}^{r} \mathrm{~d} t\right)^{1 / r}$, with the usual modification if $r=\infty$. It is a Banach space if $Y$ is a Banach space.

The outline of the paper is as follows. First, we present the discontinuous Galerkin method and the first splitting technique. In Sections 2 and 4, a priori estimates and suboptimal error estimates are derived. Section 3 contains $L^{r}$ estimates. Improved (optimal) error estimates are proved in Section 5. The second and third splitting methods are briefly presented in Section 6 . The paper ends with numerical experiments in Section 7 .

\section{Discontinuous Galerkin for Both Steps}

Problem (0.4)-(0.7) has the following weak formulation, valid a.e. on $(0, T)$ :

$$
\begin{gathered}
\forall \boldsymbol{v} \in H_{0}^{1}(\Omega)^{2}, \quad\left(\boldsymbol{u}_{t}(t), \boldsymbol{v}\right)+\mu(\nabla \boldsymbol{u}(t), \nabla \boldsymbol{v})+(\boldsymbol{u}(t) \cdot \nabla \boldsymbol{u}(t), \boldsymbol{v})-(p(t), \nabla \cdot \boldsymbol{v})=(\boldsymbol{f}(t), \boldsymbol{v}), \\
\forall q \in L_{0}^{2}(\Omega), \quad(\nabla \cdot \boldsymbol{u}(t), q)=0, \\
\boldsymbol{u}(0)=\boldsymbol{u}_{0}, \quad \text { in } \Omega .
\end{gathered}
$$

To discretize this problem, we introduce a regular family of triangulations of $\bar{\Omega}, \mathcal{E}_{h}$, consisting of triangles of maximum diameter $h$. Let $h_{E}$ denote the diameter of a triangle $E$ and $\rho_{E}$ the diameter of its inscribed circle. By regular, we mean (see Ciarlet [6]) that there exists a parameter $\zeta>0$, independent of $h$, such that

$$
\forall E \in \mathcal{E}_{h}, \quad \frac{h_{E}}{\rho_{E}}=\zeta_{E} \leq \zeta .
$$

We shall use this assumption throughout this work. We denote by $\Gamma_{h}$ the set of all edges of $\mathcal{E}_{h}$, i.e. the set of all edges in the domain $\bar{\Omega}$. Let $e$ denote a segment of $\Gamma_{h}$ shared by two triangles $E^{k}$ and $E^{l}$ of $\mathcal{E}_{h}$; we associate with $e$ a specific unit normal vector $\boldsymbol{n}_{e}$ directed from $E^{k}$ to $E^{l}$ and we define formally the jump and average of a function $\phi$ on $e$ by:

$$
[\phi]=\left.\left(\left.\phi\right|_{E^{k}}\right)\right|_{e}-\left.\left(\left.\phi\right|_{E^{l}}\right)\right|_{e}, \quad\{\phi\}=\left.\frac{1}{2}\left(\left.\phi\right|_{E^{k}}\right)\right|_{e}+\left.\frac{1}{2}\left(\left.\phi\right|_{E^{l}}\right)\right|_{e} .
$$

If $e$ is adjacent to $\partial \Omega$, then $\boldsymbol{n}_{e}$ is the unit normal $\boldsymbol{n}$ exterior to $\Omega$ and the jump and the average of $\phi$ on $e$ coincide with the trace of $\phi$ on $e$. Then, we define the spaces of discontinuous functions

$$
\begin{aligned}
& \boldsymbol{X}=\left\{\boldsymbol{v} \in L^{2}(\Omega)^{2}: \quad \forall E \in \mathcal{E}_{h},\left.\quad \boldsymbol{v}\right|_{E} \in\left(W^{2,4 / 3}(E)\right)^{2}\right\}, \\
& M=\left\{q \in L_{0}^{2}(\Omega): \quad \forall E \in \mathcal{E}_{h},\left.\quad q\right|_{E} \in W^{1,4 / 3}(E)\right\},
\end{aligned}
$$


and the broken norm, for any vector or tensor $\boldsymbol{v}$ :

$$
\|\boldsymbol{v}\|_{0, \Omega}=\left(\sum_{E \in \mathcal{E}_{h}}\|\boldsymbol{v}\|_{L^{2}(E)}^{2}\right)^{1 / 2} .
$$

We associate with the spaces $\boldsymbol{X}$ and $M$ the following norms:

$$
\begin{gathered}
\|\boldsymbol{v}\|_{X}=\left(\|\nabla \boldsymbol{v}\|_{0, \Omega}^{2}+J_{0}(\boldsymbol{v}, \boldsymbol{v})\right)^{1 / 2} \\
\|q\|_{M}=\|q\|_{L^{2}(\Omega)}
\end{gathered}
$$

where

$$
J_{0}(\boldsymbol{u}, \boldsymbol{v})=\sum_{e \in \Gamma_{h}} \frac{\sigma_{e}}{|e|} \int_{e}[\boldsymbol{u}] \cdot[\boldsymbol{v}] .
$$

Here $|e|$ denotes the measure of $e$ and $\sigma_{e}$ is a jump coefficient bounded below by a sufficiently large constant $\sigma_{0} \geq 1$ and bounded above by a constant $\sigma_{m}$, both constants being independent of $h$, but dependent on the method used. For the symmetric method, SIPG, each constant $\sigma_{e}$ is adjusted in order to guarantee ellipticity of the form $a+J_{0}$, see (1.22). For the non-symmetric method, NIPG, it is well-known that it suffices to take each constant equal to one (for instance). However, we shall see in Section 2, that because of the splitting, each constant $\sigma_{e}$ has to be adjusted in order to prove stability of the algorithm. Nevertheless, our numerical results in Section 7 tend to show that in the examples we have chosen, the error is not very sensitive to the choice of $\sigma_{e}$ when using NIPG.

On this triangulation, we define two finite-dimensional subspaces $\boldsymbol{X}_{h} \subset \boldsymbol{X}$ and $M_{h} \subset M$ :

$$
\begin{aligned}
& \boldsymbol{X}_{h}=\left\{\boldsymbol{v}_{h} \in\left(L^{2}(\Omega)\right)^{2}: \quad \forall E \in \mathcal{E}_{h}, \quad \boldsymbol{v}_{h} \in\left(\mathbb{P}_{1}(E)\right)^{2}\right\} \\
& M_{h}=\left\{q_{h} \in L_{0}^{2}(\Omega): \quad \forall E \in \mathcal{E}_{h}, \quad q_{h} \in \mathbb{P}_{0}(E)\right\}
\end{aligned}
$$

For simplicity, we derive the analysis for piecewise linear velocity and piecewise constant pressure. This is consistent with the fact that we shall use a first-order discretization in time. We could consider a higherorder approximations in space, but this would have to be matched by a higher-order approximation in time or an appropriately small time step as demonstrated in the numerical examples in Section 7 . To simplify the discussion, we shall analyze in detail the standard discontinuous symmetric method SIPG and briefly sketch the analysis for the non-symmetric method. In both methods, the incompressibility condition is enforced by means of the bilinear form $b: \boldsymbol{X} \times M \rightarrow \mathbb{R}$

$$
b(\boldsymbol{v}, p)=-\sum_{E \in \mathcal{E}_{h}} \int_{E} p \nabla \cdot \boldsymbol{v}+\sum_{e \in \Gamma_{h}} \int_{e}\{p\}[\boldsymbol{v}] \cdot \boldsymbol{n}_{e}
$$

that is simply obtained by applying Green's formula in each element to the left-hand side of (1.2). In particular if $p \in H^{1}(\Omega)$, then

$$
\forall \boldsymbol{v} \in \boldsymbol{X}, \quad b(\boldsymbol{v}, p)=\int_{\Omega} \nabla p \cdot \boldsymbol{v} .
$$

Thus, we approximate the space $\boldsymbol{V}$ defined in (0.8) by

$$
\boldsymbol{V}_{h}=\left\{\boldsymbol{v}_{h} \in \boldsymbol{X}_{h}: \forall q_{h} \in M_{h}, b\left(\boldsymbol{v}_{h}, q_{h}\right)=0\right\}
$$

Finally the nonlinear convection term $\boldsymbol{u} \cdot \nabla \boldsymbol{u}$ is approximated by the following variant of Lesaint-Raviart upwinding (see [21]) that was introduced in [14]. In theory, it is difficult to prove that it brings an improvement, 
because the Navier-Stokes equation is not purely a transport equation, but in practice, upwinding is useful when the convection is dominant. The approximation we propose is:

$$
\begin{aligned}
\forall \boldsymbol{u}, \boldsymbol{v}, \boldsymbol{w}, \boldsymbol{z} \in \boldsymbol{X}, \quad c^{\boldsymbol{z}}(\boldsymbol{u} ; \boldsymbol{v}, \boldsymbol{w})= & \sum_{E \in \mathcal{E}_{h}}\left(\int_{E}(\boldsymbol{u} \cdot \nabla \boldsymbol{v}) \cdot \boldsymbol{w}+\frac{1}{2} \int_{E}(\nabla \cdot \boldsymbol{u}) \boldsymbol{v} \cdot \boldsymbol{w}\right) \\
& -\frac{1}{2} \sum_{e \in \Gamma_{h}} \int_{e}[\boldsymbol{u}] \cdot \boldsymbol{n}_{e}\{\boldsymbol{v} \cdot \boldsymbol{w}\}+\sum_{E \in \mathcal{E}_{h}} \int_{\partial E_{-}}\left|\{\boldsymbol{u}\} \cdot \boldsymbol{n}_{E}\right|\left(\boldsymbol{v}^{\mathrm{int}}-\boldsymbol{v}^{\mathrm{ext}}\right) \cdot \boldsymbol{w}^{\mathrm{int}},
\end{aligned}
$$

where

$$
\partial E_{-}=\left\{\boldsymbol{x} \in \partial E:\{\boldsymbol{z}\} \cdot \boldsymbol{n}_{E}<0\right\}
$$

the superscript $\boldsymbol{z}$ denotes the dependence of $\partial E_{-}$on $\boldsymbol{z}$ and the superscript int (resp. ext) refers to the trace of the function on a side of $E$ coming from the interior of $E$ (resp. coming from the exterior of $E$ on that side). When the side of $E$ belongs to $\partial \Omega$, the convention is the same as for defining the jump and average, i.e., the jump and average coincide with the trace of the function. Note that $c^{\boldsymbol{z}}(\boldsymbol{u} ; \boldsymbol{v}, \boldsymbol{w})$ can also be written as

$$
c^{\boldsymbol{z}}(\boldsymbol{u} ; \boldsymbol{v}, \boldsymbol{w})=\sum_{E \in \mathcal{E}_{h}}\left(\int_{E}(\boldsymbol{u} \cdot \nabla \boldsymbol{v}) \cdot \boldsymbol{w}+\int_{\partial E_{-}}\left|\{\boldsymbol{u}\} \cdot \boldsymbol{n}_{E}\right|\left(\boldsymbol{v}^{\mathrm{int}}-\boldsymbol{v}^{\mathrm{ext}}\right) \cdot \boldsymbol{w}^{\mathrm{int}}\right)-\frac{1}{2} b(\boldsymbol{u}, \boldsymbol{v} \cdot \boldsymbol{w}) .
$$

Thus if $\boldsymbol{v}$ is continuous in $\Omega$ or belongs to $\left(H^{1}(\Omega)\right)^{2}$, we have

$$
c(\boldsymbol{u} ; \boldsymbol{v}, \boldsymbol{w})=\sum_{E \in \mathcal{E}_{h}} \int_{E}(\boldsymbol{u} \cdot \nabla \boldsymbol{v}) \cdot \boldsymbol{w}-\frac{1}{2} b(\boldsymbol{u}, \boldsymbol{v} \cdot \boldsymbol{w}) .
$$

The superscript $\boldsymbol{z}$ is dropped since the integral on $\partial E_{-}$disappears. It is proven in [14] that for all $\boldsymbol{u}, \boldsymbol{v}, \boldsymbol{w} \in \boldsymbol{X}$ we have

$$
c^{\left.u_{(u} ; \boldsymbol{v}, \boldsymbol{w}\right)}=-\bar{c} \boldsymbol{u}_{(\boldsymbol{u} ; \boldsymbol{w}, \boldsymbol{v})}
$$

where

$$
\begin{aligned}
{ }_{\bar{c}} \boldsymbol{u}(\boldsymbol{u} ; \boldsymbol{w}, \boldsymbol{v}):=\sum_{E \in \mathcal{E}_{h}}\left(\int_{E}(\boldsymbol{u} \cdot \nabla \boldsymbol{w}) \cdot \boldsymbol{v}\right. & \left.+\frac{1}{2} \int_{E}(\nabla \cdot \boldsymbol{u}) \boldsymbol{w} \cdot \boldsymbol{v}\right)-\frac{1}{2} \sum_{e \in\left(\Gamma_{h}\right) \backslash(\partial \Omega)} \int_{e}[\boldsymbol{u}] \cdot \boldsymbol{n}_{e}\{\boldsymbol{v} \cdot \boldsymbol{w}\} \\
& +\sum_{E \in \mathcal{E}_{h}} \int_{\left(\partial E_{-}\right) \backslash(\partial \Omega)}\left|\{\boldsymbol{u}\} \cdot \boldsymbol{n}_{E}\right|\left(\boldsymbol{w}^{\mathrm{int}}-\boldsymbol{w}^{\mathrm{ext}}\right) \cdot \boldsymbol{v}^{\mathrm{ext}}-\frac{1}{2} \sum_{e \in \partial \Omega} \int_{e}\left|\boldsymbol{u} \cdot \boldsymbol{n}_{e}\right| \boldsymbol{v} \cdot \boldsymbol{w}
\end{aligned}
$$

This implies that for all $\boldsymbol{u}, \boldsymbol{v} \in \boldsymbol{X}$,

$$
c^{\boldsymbol{u}}(\boldsymbol{u} ; \boldsymbol{v}, \boldsymbol{v})=\frac{1}{2} \sum_{E \in \mathcal{E}_{h}} \int_{\partial E_{-}}\left|\{\boldsymbol{u}\} \cdot \boldsymbol{n}_{E}\right|\|[\boldsymbol{v}]\|^{2}+\frac{1}{2} \sum_{E \in \mathcal{E}_{h}} \int_{\left(\partial E_{-}\right) \cap(\partial \Omega)}\left|\boldsymbol{u} \cdot \boldsymbol{n}_{E}\right|\|\boldsymbol{v}\|^{2},
$$

where $\|\cdot\|$ denotes the Euclidean norm.

\subsection{Approximation with SIPG}

In SIPG, the diffusion operator is approximated by the bilinear form $a: X \times \boldsymbol{X} \rightarrow \mathbb{R}$

$$
a(\boldsymbol{u}, \boldsymbol{v})=\sum_{E \in \mathcal{E}_{h}} \int_{E} \nabla \boldsymbol{u}: \nabla \boldsymbol{v}-\sum_{e \in \Gamma_{h}} \int_{e}\{\nabla \boldsymbol{u}\} \boldsymbol{n}_{e} \cdot[\boldsymbol{v}]-\sum_{e \in \Gamma_{h}} \int_{e}\{\nabla \boldsymbol{v}\} \boldsymbol{n}_{e} \cdot[\boldsymbol{u}] .
$$


Considering this form $a$, it will be useful to introduce another mesh-dependent norm:

$$
\forall \boldsymbol{v} \in \boldsymbol{X}, \quad \llbracket \boldsymbol{v} \|=\left(\|\boldsymbol{v}\|_{X}^{2}+\sum_{e \in \Gamma_{h}}|e|\left\|\{\nabla \boldsymbol{v}\} \cdot \boldsymbol{n}_{e}\right\|_{L^{2}(e)}^{2}\right)^{1 / 2} .
$$

Then, we have

$$
\forall \boldsymbol{u}, \boldsymbol{v} \in \boldsymbol{X}, \quad|a(\boldsymbol{u}, \boldsymbol{v})| \leq \llbracket \boldsymbol{u} \| \llbracket \boldsymbol{v} \rrbracket .
$$

Note that when $\boldsymbol{v} \in \boldsymbol{X}_{h}$, the equivalence of norms in finite-dimensional spaces on the reference element implies that there exists a constant $C$ independent of $h$ such that

$$
\forall \boldsymbol{v} \in \boldsymbol{X}_{h}, \quad\left(\sum_{e \in \Gamma_{h}}|e|\left\|\{\nabla \boldsymbol{v}\} \cdot \boldsymbol{n}_{e}\right\|_{L^{2}(e)}^{2}\right)^{1 / 2} \leq C\|\nabla \boldsymbol{v}\|_{0, \Omega} .
$$

As far as the ellipticity of the form $a+J_{0}$ is concerned, it is established in [30] that, if the coefficients $\sigma_{e}$ are sufficiently large but independent of $h$, there exists a constant $K>0$, also independent of $h$, such that

$$
\forall \boldsymbol{v}_{h} \in \boldsymbol{X}_{h}, \quad a\left(\boldsymbol{v}_{h}, \boldsymbol{v}_{h}\right)+J_{0}\left(\boldsymbol{v}_{h}, \boldsymbol{v}_{h}\right) \geq K\left\|\boldsymbol{v}_{h}\right\|_{X}^{2}
$$

In the sequel, we shall always assume that (1.22) holds so that $a+J_{0}$ is elliptic. As far as the inf-sup condition is concerned, it is proven in [14] that the pair of spaces defined by (1.10), (1.11) satisfies a uniform discrete inf-sup condition. More precisely, with the space

$$
\overline{\boldsymbol{X}}_{h}=\left\{\boldsymbol{v}_{h} \in \boldsymbol{X}_{h}: \forall e \in \Gamma_{h}, \quad \int_{e}\left[\boldsymbol{v}_{h}\right]=\mathbf{0}\right\},
$$

we have the following result:

Lemma 1.1. There exists a constant $\beta^{*}>0$, independent of $h$, such that

$$
\inf _{p_{h} \in M_{h}} \sup _{\boldsymbol{v}_{h} \in \overline{\boldsymbol{X}}_{h}} \frac{b\left(\boldsymbol{v}_{h}, p_{h}\right)}{\left\|\boldsymbol{v}_{h}\right\|_{X}\left\|p_{h}\right\|_{M}} \geq \beta^{*} .
$$

Discretization with respect to time is done on a uniform subdivision of the interval $[0, T]$. Let $N \geq 2$ be an integer, $\Delta t=\frac{T}{N}$ and $t^{j}=j \Delta t, 0 \leq j \leq N$. Since the approximation both in space and time are of order one, we assume that $h$ and $\Delta t$ are of the same order, i.e. there exist constants $\gamma_{0}$ and $\gamma_{1}$ independent of $h$ and $\Delta t$ such that

$$
\gamma_{0} \Delta t \leq h \leq \gamma_{1} \Delta t
$$

The discrete scheme consists of two steps. First, knowing $\boldsymbol{U}^{j} \in \boldsymbol{V}_{h}$, find $\tilde{\boldsymbol{U}}^{j+1} \in \boldsymbol{X}_{h}$ solution of

$$
\forall \boldsymbol{v}_{h} \in \boldsymbol{X}_{h}, \quad \frac{1}{\Delta t}\left(\tilde{\boldsymbol{U}}^{j+1}-\boldsymbol{U}^{j}, \boldsymbol{v}_{h}\right)+\mu\left(a\left(\tilde{\boldsymbol{U}}^{j+1}, \boldsymbol{v}_{h}\right)+J_{0}\left(\tilde{\boldsymbol{U}}^{j+1}, \boldsymbol{v}_{h}\right)\right)+c^{\boldsymbol{U}^{j}}\left(\boldsymbol{U}^{j} ; \tilde{\boldsymbol{U}}^{j+1}, \boldsymbol{v}_{h}\right)=\left(\check{\boldsymbol{f}}^{j+1}, \boldsymbol{v}_{h}\right) .
$$

Second, find $\boldsymbol{U}^{j+1} \in \boldsymbol{X}_{h}$ and $P^{j+1} \in M_{h}$, solution of

$$
\begin{gathered}
\forall \boldsymbol{v}_{h} \in \boldsymbol{X}_{h}, \quad \frac{1}{\Delta t}\left(\boldsymbol{U}^{j+1}-\tilde{\boldsymbol{U}}^{j+1}, \boldsymbol{v}_{h}\right)+\mu\left(a\left(\boldsymbol{U}^{j+1}-\tilde{\boldsymbol{U}}^{j+1}, \boldsymbol{v}_{h}\right)+J_{0}\left(\boldsymbol{U}^{j+1}-\tilde{\boldsymbol{U}}^{j+1}, \boldsymbol{v}_{h}\right)\right)+b\left(\boldsymbol{v}_{h}, P^{j+1}\right)=0 \\
\forall q_{h} \in M_{h}, \quad b\left(\boldsymbol{U}^{j+1}, q_{h}\right)=0 .
\end{gathered}
$$


At time $t=0, \boldsymbol{U}^{0}$ is a suitable approximation of $\boldsymbol{u}_{0}$ that we specify later. The term $\check{\boldsymbol{f}}^{j}$ denotes an appropriate approximation of $\boldsymbol{f}$ at time $t^{j}$. To simplify the analysis, we choose

$$
\check{\boldsymbol{f}}^{j}=\frac{1}{\Delta t} \int_{t^{j-1}}^{t^{j}} \boldsymbol{f}
$$

but this is only a matter of convenience. As far as existence is concerned, given $\boldsymbol{U}^{j},(1.26)$ has a unique solution owing to the ellipticity property $(1.22)$ and the positivity $(1.18)$ of $c$. Similarly, given $\tilde{\boldsymbol{U}}^{j+1},(1.27),(1.28)$ has a unique solution owing to the ellipticity property (1.22) and the inf-sup condition (1.24). By summing the two steps, the consistency of the scheme follows from the following lemma. We skip the proof, which is straightforward.

Lemma 1.2. Formally, the solution $(\boldsymbol{u}, p)$ of $(0.4)-(0.7)$ satisfies a.e. on $(0, T)$ :

$$
\forall \boldsymbol{v}_{h} \in \boldsymbol{X}_{h}, \quad\left(\boldsymbol{u}_{t}, \boldsymbol{v}_{h}\right)+\mu\left(a\left(\boldsymbol{u}, \boldsymbol{v}_{h}\right)+J_{0}\left(\boldsymbol{u}, \boldsymbol{v}_{h}\right)\right)+c\left(\boldsymbol{u} ; \boldsymbol{u}, \boldsymbol{v}_{h}\right)+b\left(\boldsymbol{v}_{h}, p\right)=\left(\boldsymbol{f}, \boldsymbol{v}_{h}\right) .
$$

Now we recall some approximation properties of the spaces $\boldsymbol{X}_{h}$ and $M_{h}$. For $\boldsymbol{X}_{h}$, let $R_{h} \in \mathcal{L}\left(H^{1}(\Omega)^{2} ; \boldsymbol{X}_{h}\right)$ be the operator defined by

$$
\forall \boldsymbol{v} \in H^{1}(\Omega)^{2}, \forall e \in \Gamma_{h}, \quad \int_{e}\left(R_{h} \boldsymbol{v}-\boldsymbol{v}\right)=\mathbf{0} .
$$

It is easy to see that (1.30) defines a unique function $R_{h} \boldsymbol{v} \in \boldsymbol{X}_{h}$ (see [8]) and implies that

$$
\begin{gathered}
\forall \boldsymbol{v} \in H^{1}(\Omega)^{2}, \quad \int_{E} \nabla \cdot\left(R_{h} \boldsymbol{v}-\boldsymbol{v}\right)=0, \\
\forall \boldsymbol{v} \in H_{0}^{1}(\Omega)^{2}, \quad \forall e \in \Gamma_{h}, \quad \int_{e}\left[R_{h} \boldsymbol{v}\right]=\mathbf{0} .
\end{gathered}
$$

Thus, we have

$$
\forall \boldsymbol{v} \in H_{0}^{1}(\Omega)^{2}, \quad \forall q_{h} \in M_{h}, \quad b\left(\boldsymbol{v}-R_{h} \boldsymbol{v}, q_{h}\right)=0 .
$$

Furthermore, since $R_{h}$ preserves the polynomials of $\mathbb{P}_{1}$ in each element, it satisfies the error bounds:

$$
\forall E \in \mathcal{E}_{h}, \forall s \in[1,2], \forall r \geq 2, \forall \boldsymbol{v} \in W^{s, r}(E)^{2}, m=0,1,\left|\boldsymbol{v}-R_{h} \boldsymbol{v}\right|_{W^{m, r}(E)} \leq C h_{E}^{s-m}|\boldsymbol{v}|_{W^{s, r}(E)} .
$$

For $M_{h}$, let $r_{h} \in \mathcal{L}\left(L_{0}^{2}(\Omega) ; M_{h}\right)$ be defined in each $E \in \mathcal{E}_{h}$ by:

$$
\forall E \in \mathcal{E}_{h}, \quad \int_{E}\left(r_{h} q-q\right)=0
$$

Then

$$
\forall q \in H^{s}(E), \quad \forall s \in[0,1], \quad\left\|q-r_{h} q\right\|_{L^{2}(E)} \leq C h_{E}^{s}|q|_{H^{s}(E)} .
$$

From (1.32) and (1.34) with $s=m=1$ and $r=2$, we easily derive the next lemma.

Lemma 1.3. The operator $R_{h}$ satisfies the following stability property: there exists a constant $C$ independent of $h$ such that,

$$
\forall \boldsymbol{u} \in H_{0}^{1}(\Omega)^{2}, \quad\left\|R_{h} \boldsymbol{u}\right\|_{X} \leq C|\boldsymbol{u}|_{H^{1}(\Omega)} .
$$

We have the following consistency error for $a$ :

Lemma 1.4. There exists a constant $C$, independent of $h$, such that for all $\boldsymbol{u}$ in $\left(H^{2}(\Omega) \cap H_{0}^{1}(\Omega)\right)^{2}$ and all $\boldsymbol{v}_{h}$ in $\boldsymbol{X}_{h}$ :

$$
\left|a\left(\boldsymbol{u}-R_{h} \boldsymbol{u}, \boldsymbol{v}_{h}\right)\right| \leq C h|\boldsymbol{u}|_{H^{2}(\Omega)}\left\|\boldsymbol{v}_{h}\right\|_{X}
$$


Proof. In view of (1.20) and (1.21) it suffices to prove that

$$
\forall \boldsymbol{u} \in\left(H^{2}(\Omega) \cap H_{0}^{1}(\Omega)\right)^{2}, \quad \llbracket \boldsymbol{u}-R_{h} \boldsymbol{u} \| \leq C h|\boldsymbol{u}|_{H^{2}(\Omega)} .
$$

This follows easily from (1.34).

Remark 1.5. The proposed splitting technique satisfies the compatibility condition of zero accuracy described in [10] where piecewise discontinuous linears are used in a discontinuous Galerkin transport scheme. In other words, constants are reproduced when an approximate velocity defined by (1.27), (1.28) is used in transport. The compatibility condition is:

$$
\forall j>0, \quad \forall E \in \mathcal{E}_{h}, \quad \int_{\partial E}\left\{\boldsymbol{U}^{j}\right\} \cdot \boldsymbol{n}_{E}=0
$$

This condition follows immediately from (1.28).

\subsection{Approximation with NIPG}

In NIPG, the form $a$ is replaced by

$$
a(\boldsymbol{u}, \boldsymbol{v})=\sum_{E \in \mathcal{E}_{h}} \int_{E} \nabla \boldsymbol{u}: \nabla \boldsymbol{v}-\sum_{e \in \Gamma_{h}} \int_{e}\{\nabla \boldsymbol{u}\} \boldsymbol{n}_{e} \cdot[\boldsymbol{v}]+\sum_{e \in \Gamma_{h}} \int_{e}\{\nabla \boldsymbol{v}\} \boldsymbol{n}_{e} \cdot[\boldsymbol{u}],
$$

and for the moment, we take each constant $\sigma_{e} \geq 1$ arbitrary. All the other terms are unchanged and the formulation of the discrete problem is given, with this new form $a$, by (1.26)-(1.28). Clearly, all the properties listed above are preserved and (1.22) is improved since

$$
\forall \boldsymbol{v}_{h} \in \boldsymbol{X}_{h}, \quad a\left(\boldsymbol{v}_{h}, \boldsymbol{v}_{h}\right)+J_{0}\left(\boldsymbol{v}_{h}, \boldsymbol{v}_{h}\right)=\left\|\boldsymbol{v}_{h}\right\|_{X}^{2}
$$

\section{A PRIORI ESTIMATES}

In this section, we prove that the scheme (1.26)-(1.28) is unconditionally stable. The proof uses the discrete Poincaré inequality (3.14) in the particular case where $r=2$.

\subsection{Approximation with SIPG}

Proposition 2.1. If the ellipticity (1.22) holds, the sequences $\boldsymbol{U}^{j}$ and $\tilde{\boldsymbol{U}}^{j}$ defined by (1.26)-(1.28) satisfy the following a priori estimate:

$$
\begin{array}{r}
\left\|\boldsymbol{U}^{N}\right\|_{L^{2}(\Omega)}^{2}+\sum_{j=0}^{N-1}\left(\left\|\boldsymbol{U}^{j+1}-\tilde{\boldsymbol{U}}^{j+1}\right\|_{L^{2}(\Omega)}^{2}+\left\|\tilde{\boldsymbol{U}}^{j+1}-\boldsymbol{U}^{j}\right\|_{L^{2}(\Omega)}^{2}\right)+\sum_{j=0}^{N-1} \Delta t \sum_{E \in \mathcal{E}_{h}} \int_{\partial E_{-}}\left|\left\{\boldsymbol{U}^{j}\right\} \cdot \boldsymbol{n}_{E}\right|\left\|\left[\tilde{\boldsymbol{U}}^{j+1}\right]\right\|^{2} \\
+\sum_{j=0}^{N-1} \Delta t \sum_{E \in \mathcal{E}_{h}} \int_{\left(\partial E_{-}\right) \cap(\partial \Omega)}\left|\boldsymbol{U}^{j} \cdot \boldsymbol{n}_{E}\right|\left\|\tilde{\boldsymbol{U}}^{j+1}\right\|^{2}+\mu K \Delta t\left(\sum_{j=1}^{N}\left\|\boldsymbol{U}^{j}\right\|_{X}^{2}+\frac{1}{2} \sum_{j=1}^{N}\left\|\tilde{\boldsymbol{U}}^{j}\right\|_{X}^{2}+\sum_{j=1}^{N}\left\|\boldsymbol{U}^{j}-\tilde{\boldsymbol{U}}^{j}\right\|_{X}^{2}\right) \\
\leq\left\|\boldsymbol{U}^{0}\right\|_{L^{2}(\Omega)}^{2}+\frac{2 C_{0}^{2}}{\mu K} \sum_{j=1}^{N} \Delta t\left\|\check{\boldsymbol{f}}^{j}\right\|_{L^{2}(\Omega)}^{2}, \quad(2.1)
\end{array}
$$

where $K$ is the constant of (1.22) and $C_{0}$ is the constant of (3.14) with $r=2$. 
Proof. First taking $\boldsymbol{v}=\tilde{\boldsymbol{U}}^{j+1}$ in (1.26) and using (1.18), we obtain:

$$
\begin{aligned}
& \frac{1}{2 \Delta t}\left(\left\|\tilde{\boldsymbol{U}}^{j+1}\right\|_{L^{2}(\Omega)}^{2}-\left\|\boldsymbol{U}^{j}\right\|_{L^{2}(\Omega)}^{2}+\left\|\tilde{\boldsymbol{U}}^{j+1}-\boldsymbol{U}^{j}\right\|_{L^{2}(\Omega)}^{2}\right)+\mu\left(a\left(\tilde{\boldsymbol{U}}^{j+1}, \tilde{\boldsymbol{U}}^{j+1}\right)+J_{0}\left(\tilde{\boldsymbol{U}}^{j+1}, \tilde{\boldsymbol{U}}^{j+1}\right)\right) \\
& +\frac{1}{2} \sum_{E \in \mathcal{E}_{h}} \int_{\partial E_{-}}\left|\left\{\boldsymbol{U}^{j}\right\} \cdot \boldsymbol{n}_{E}\right|\left\|\left[\tilde{\boldsymbol{U}}^{j+1}\right]\right\|^{2}+\frac{1}{2} \sum_{E \in \mathcal{E}_{h}} \int_{\left(\partial E_{-}\right) \cap(\partial \Omega)}\left|\boldsymbol{U}^{j} \cdot \boldsymbol{n}_{E}\right|\left\|\tilde{\boldsymbol{U}}^{j+1}\right\|^{2}=\left(\tilde{\boldsymbol{f}}^{j+1}, \tilde{\boldsymbol{U}}^{j+1}\right) .
\end{aligned}
$$

Next taking $\boldsymbol{v}=\boldsymbol{U}^{j+1}$ in (1.27) and using the symmetry of $a$ and (1.28), we obtain

$$
\begin{aligned}
\frac{1}{2 \Delta t}\left(\left\|\boldsymbol{U}^{j+1}\right\|_{L^{2}(\Omega)}^{2}-\right. & \left.\left\|\tilde{\boldsymbol{U}}^{j+1}\right\|_{L^{2}(\Omega)}^{2}+\left\|\boldsymbol{U}^{j+1}-\tilde{\boldsymbol{U}}^{j+1}\right\|_{L^{2}(\Omega)}^{2}\right) \\
+ & \frac{\mu}{2}\left(a\left(\boldsymbol{U}^{j+1}, \boldsymbol{U}^{j+1}\right)-a\left(\tilde{\boldsymbol{U}}^{j+1}, \tilde{\boldsymbol{U}}^{j+1}\right)+a\left(\boldsymbol{U}^{j+1}-\tilde{\boldsymbol{U}}^{j+1}, \boldsymbol{U}^{j+1}-\tilde{\boldsymbol{U}}^{j+1}\right)\right) \\
& +\frac{\mu}{2}\left(J_{0}\left(\boldsymbol{U}^{j+1}, \boldsymbol{U}^{j+1}\right)-J_{0}\left(\tilde{\boldsymbol{U}}^{j+1}, \tilde{\boldsymbol{U}}^{j+1}\right)+J_{0}\left(\boldsymbol{U}^{j+1}-\tilde{\boldsymbol{U}}^{j+1}, \boldsymbol{U}^{j+1}-\tilde{\boldsymbol{U}}^{j+1}\right)\right)=0 .
\end{aligned}
$$

Summing (2.2) and (2.3), and using the ellipticity (1.22), we obtain

$$
\begin{aligned}
& \frac{1}{2 \Delta t}\left(\left\|\boldsymbol{U}^{j+1}\right\|_{L^{2}(\Omega)}^{2}-\left\|\boldsymbol{U}^{j}\right\|_{L^{2}(\Omega)}^{2}+\right.\left.\left\|\boldsymbol{U}^{j+1}-\tilde{\boldsymbol{U}}^{j+1}\right\|_{L^{2}(\Omega)}^{2}+\left\|\tilde{\boldsymbol{U}}^{j+1}-\boldsymbol{U}^{j}\right\|_{L^{2}(\Omega)}^{2}\right) \\
&+\frac{1}{2} \sum_{E \in \mathcal{E}_{h}} \int_{\partial E_{-}}\left|\left\{\boldsymbol{U}^{j}\right\} \cdot \boldsymbol{n}_{E}\right|\left\|\left[\tilde{\boldsymbol{U}}^{j+1}\right]\right\|^{2}+\frac{1}{2} \sum_{E \in \mathcal{E}_{h}} \int_{\left(\partial E_{-}\right) \cap(\partial \Omega)}\left|\boldsymbol{U}^{j} \cdot \boldsymbol{n}_{E}\right|\left\|\tilde{\boldsymbol{U}}^{j+1}\right\|^{2} \\
&+\frac{\mu K}{2}\left(\left\|\boldsymbol{U}^{j+1}\right\|_{X}^{2}+\left\|\tilde{\boldsymbol{U}}^{j+1}\right\|_{X}^{2}+\left\|\boldsymbol{U}^{j+1}-\tilde{\boldsymbol{U}}^{j+1}\right\|_{X}^{2}\right) \leq\left|\left(\tilde{\boldsymbol{f}}^{j+1}, \tilde{\boldsymbol{U}}^{j+1}\right)\right| .
\end{aligned}
$$

We now derive an estimate that is proportional to $T$ and essentially inversely proportional to the viscosity. First from (3.14), the right hand-side of (2.4) is bounded as follows, for any $\epsilon>0$ :

$$
\left|\left(\check{\boldsymbol{f}}^{j+1}, \tilde{\boldsymbol{U}}^{j+1}\right)\right| \leq\left\|\check{\boldsymbol{f}}^{j+1}\right\|_{L^{2}(\Omega)}\left\|\tilde{\boldsymbol{U}}^{j+1}\right\|_{L^{2}(\Omega)} \leq C_{0}\left\|\check{\boldsymbol{f}}^{j+1}\right\|_{L^{2}(\Omega)}\left\|\tilde{\boldsymbol{U}}^{j+1}\right\|_{X} \leq \frac{\epsilon}{2}\left\|\tilde{\boldsymbol{U}}^{j+1}\right\|_{X}^{2}+\frac{C_{0}^{2}}{2 \epsilon}\left\|\check{\boldsymbol{f}}^{j+1}\right\|_{L^{2}(\Omega)}^{2} .
$$

Choose $\epsilon=\frac{\mu K}{2}$, then (2.4) becomes

$$
\begin{aligned}
& \frac{1}{2 \Delta t}\left(\left\|\boldsymbol{U}^{j+1}\right\|_{L^{2}(\Omega)}^{2}-\right.\left\|\boldsymbol{U}^{j}\right\|_{L^{2}(\Omega)}^{2}+ \\
&\left.+\frac{1}{2} \sum_{E \in \mathcal{E}_{h}} \int_{\partial E_{-}}\left|\left\{\boldsymbol{U}^{j}\right\} \cdot \boldsymbol{n}_{E}\right|\left\|\left[\tilde{\boldsymbol{U}}^{j+1}\right]\right\|^{2}+\frac{1}{2} \sum_{E \in \mathcal{E}_{h}} \int_{(\partial E-)} \tilde{\boldsymbol{U}}^{j+1}\left\|_{L^{2}(\Omega)}^{2}+\right\| \tilde{\boldsymbol{U}}^{j+1}-\boldsymbol{U}^{j} \|_{L^{2}(\Omega)}^{2}\right) \\
&+\frac{\mu K}{2}\left(\left\|\boldsymbol{n}_{E} \mid\right\| \tilde{\boldsymbol{U}}^{j+1}\left\|_{X}^{2}+\frac{1}{2}\right\| \tilde{\boldsymbol{U}}^{j+1}\left\|_{X}^{2}+\right\| \boldsymbol{U}^{j+1}-\tilde{\boldsymbol{U}}^{j+1} \|_{X}^{2}\right) \leq \frac{C_{0}^{2}}{\mu K}\left\|\check{\boldsymbol{f}}^{j+1}\right\|_{L^{2}(\Omega)}^{2} .
\end{aligned}
$$

The result follows by multiplying by $2 \Delta t$ and summing from $j=0$ to $j=N-1$.

Proposition 2.1 has in particular the following corollary.

Corollary 2.2. If the ellipticity (1.22) holds, the quantities:

$$
\sup _{j}\left\|\boldsymbol{U}^{j}\right\|_{L^{2}(\Omega)}, \sup _{j}\left\|\tilde{\boldsymbol{U}}^{j}\right\|_{L^{2}(\Omega)}, \sqrt{\mu K}\left(\sum_{j=1}^{N} \Delta t\left\|\boldsymbol{U}^{j}\right\|_{X}^{2}\right)^{1 / 2}, \sqrt{\frac{\mu K}{2}}\left(\sum_{j=1}^{N} \Delta t\left\|\tilde{\boldsymbol{U}}^{j}\right\|_{X}^{2}\right)^{1 / 2}
$$


are all bounded by

$$
\left(\left\|\boldsymbol{U}^{0}\right\|_{L^{2}(\Omega)}^{2}+\frac{2 C_{0}^{2}}{\mu K} \sum_{j=1}^{N} \Delta t\left\|\check{\boldsymbol{f}}^{j}\right\|_{L^{2}(\Omega)}^{2}\right)^{1 / 2}
$$

\subsection{Approximation with NIPG}

The scheme (1.26)-(1.28) is also unconditionally stable for NIPG provided each constant $\sigma_{e}$ is sufficiently large, but independent of $h$. More precisely, we assume that each $\sigma_{e}$ is chosen so that:

$$
\forall \boldsymbol{v} \in \boldsymbol{X}_{h}, \quad \sum_{e \in \Gamma_{h}} \frac{|e|}{\sigma_{e}} \int_{e}\left\|\{\nabla \boldsymbol{v}\} \boldsymbol{n}_{e}\right\|^{2} \leq \frac{1}{2}\|\nabla \boldsymbol{v}\|_{0, \Omega}^{2} .
$$

It is easy to check that $(2.5)$ holds provided that

$$
\forall e \in \Gamma_{h}, \quad \sigma_{0} \leq \sigma_{e} \leq \sigma_{m}
$$

for $\sigma_{0} \geq 1$ and $\sigma_{m}$ both independent of $e$ and $h$ (but possibly different from the constants of SIPG). Then Lemma 2.1 is replaced by

Proposition 2.3. Assume that (2.5) holds. Then, the sequences $\boldsymbol{U}^{j}$ and $\tilde{\boldsymbol{U}}^{j}$ defined by (1.26)-(1.28), with the form a defined by (1.39), satisfy the following a priori estimate:

$$
\begin{array}{r}
\left\|\boldsymbol{U}^{N}\right\|_{L^{2}(\Omega)}^{2}+\sum_{j=0}^{N-1}\left(\left\|\boldsymbol{U}^{j+1}-\tilde{\boldsymbol{U}}^{j+1}\right\|_{L^{2}(\Omega)}^{2}+\left\|\tilde{\boldsymbol{U}}^{j+1}-\boldsymbol{U}^{j}\right\|_{L^{2}(\Omega)}^{2}\right) \\
+\sum_{j=0}^{N-1} \Delta t \sum_{E \in \mathcal{E}_{h}} \int_{\left(\partial E_{-}\right) \cap(\partial \Omega)}\left|\sum_{E \in \mathcal{E}_{h}} \int_{\partial E_{-}}\right|\left\{\boldsymbol{U}^{j}\right\} \cdot \boldsymbol{n}_{E} \mid\left\|\left[\tilde{\boldsymbol{U}}^{j+1}\right]\right\| \|^{2} \\
+\tilde{\boldsymbol{U}}^{j+1} \|^{2}+\frac{\mu}{2} \sum_{j=1}^{N} \Delta t\left(\left\|\boldsymbol{U}^{j}\right\|_{X}^{2}+\left\|\tilde{\boldsymbol{U}}^{j}\right\|_{X}^{2}\right) \\
\leq\left\|\boldsymbol{U}^{0}\right\|_{L^{2}(\Omega)}^{2}+\frac{2 C_{0}^{2}}{\mu} \sum_{j=1}^{N} \Delta t\left\|\check{\boldsymbol{f}}^{j}\right\|_{L^{2}(\Omega)}^{2},
\end{array}
$$

where $C_{0}$ is the constant of (3.14) with $r=2$.

Proof. As in Lemma 2.1, we start with (2.2), but in (1.27) we cannot use the symmetry of $a$, so instead of (2.3), we have:

$$
\begin{aligned}
\frac{1}{2 \Delta t}\left(\left\|\boldsymbol{U}^{j+1}\right\|_{L^{2}(\Omega)}^{2}-\left\|\tilde{\boldsymbol{U}}^{j+1}\right\|_{L^{2}(\Omega)}^{2}\right. & \left.+\left\|\boldsymbol{U}^{j+1}-\tilde{\boldsymbol{U}}^{j+1}\right\|_{L^{2}(\Omega)}^{2}\right) \\
+ & \frac{\mu}{2}\left(\left\|\boldsymbol{U}^{j+1}\right\|_{X}^{2}-\left\|\tilde{\boldsymbol{U}}^{j+1}\right\|_{X}^{2}+\left\|\boldsymbol{U}^{j+1}-\tilde{\boldsymbol{U}}^{j+1}\right\|_{X}^{2}\right) \\
& +\mu\left(\sum_{e \in \Gamma_{h}} \int_{e}\left\{\nabla \tilde{\boldsymbol{U}}^{j+1}\right\} \boldsymbol{n}_{e} \cdot\left[\boldsymbol{U}^{j+1}\right]-\sum_{e \in \Gamma_{h}} \int_{e}\left\{\nabla \boldsymbol{U}^{j+1}\right\} \boldsymbol{n}_{e} \cdot\left[\tilde{\boldsymbol{U}}^{j+1}\right]\right)=0
\end{aligned}
$$


and we must find an upper bound for this last factor of $\mu$. This term can be written:

$$
\begin{aligned}
\sum_{e \in \Gamma_{h}} \int_{e}\left\{\nabla \tilde{\boldsymbol{U}}^{j+1}\right\} \boldsymbol{n}_{e} \cdot\left[\boldsymbol{U}^{j+1}\right]-\sum_{e \in \Gamma_{h}} \int_{e}\left\{\nabla \boldsymbol{U}^{j+1}\right\} \boldsymbol{n}_{e} \cdot\left[\tilde{\boldsymbol{U}}^{j+1}\right]=\sum_{e \in \Gamma_{h}} \int_{e}\left\{\nabla\left(\tilde{\boldsymbol{U}}^{j+1}-\boldsymbol{U}^{j+1}\right)\right\} \boldsymbol{n}_{e} \cdot\left[\boldsymbol{U}^{j+1}\right] \\
+\sum_{e \in \Gamma_{h}} \int_{e}\left\{\nabla \boldsymbol{U}^{j+1}\right\} \boldsymbol{n}_{e} \cdot\left[\boldsymbol{U}^{j+1}-\tilde{\boldsymbol{U}}^{j+1}\right] \\
\leq \sum_{e \in \Gamma_{h}} \frac{|e|}{\sigma_{e}}\left\|\left\{\nabla\left(\tilde{\boldsymbol{U}}^{j+1}-\boldsymbol{U}^{j+1}\right)\right\} \boldsymbol{n}_{e}\right\|_{L^{2}(e)}^{2}+\frac{1}{4} \sum_{e \in \Gamma_{h}} \frac{\sigma_{e}}{|e|}\left\|\left[\boldsymbol{U}^{j+1}\right]\right\|_{L^{2}(e)}^{2} \\
+\frac{1}{2} \sum_{e \in \Gamma_{h}} \frac{|e|}{\sigma_{e}}\left\|\left\{\nabla \boldsymbol{U}^{j+1}\right\} \boldsymbol{n}_{e}\right\|_{L^{2}(e)}^{2}+\frac{1}{2} \sum_{e \in \Gamma_{h}} \frac{\sigma_{e}}{|e|}\left\|\left[\boldsymbol{U}^{j+1}-\tilde{\boldsymbol{U}}^{j+1}\right]\right\|_{L^{2}(e)}^{2} \\
\leq \frac{1}{4}\left\|\boldsymbol{U}^{j+1}\right\|_{X}^{2}+\frac{1}{2}\left\|\tilde{\boldsymbol{U}}^{j+1}-\boldsymbol{U}^{j+1}\right\|_{X}^{2}
\end{aligned}
$$

where we have used (2.5) in the last inequality. Then substituting this bound into (2.7) and adding (2.2), we derive

$$
\begin{aligned}
\frac{1}{2 \Delta t}\left(\left\|\boldsymbol{U}^{j+1}\right\|_{L^{2}(\Omega)}^{2}-\right. & \left.\left\|\boldsymbol{U}^{j}\right\|_{L^{2}(\Omega)}^{2}+\left\|\tilde{\boldsymbol{U}}^{j+1}-\boldsymbol{U}^{j}\right\|_{L^{2}(\Omega)}^{2}+\left\|\boldsymbol{U}^{j+1}-\tilde{\boldsymbol{U}}^{j+1}\right\|_{L^{2}(\Omega)}^{2}\right) \\
& +\frac{\mu}{2}\left(\frac{1}{2}\left\|\boldsymbol{U}^{j+1}\right\|_{X}^{2}+\left\|\tilde{\boldsymbol{U}}^{j+1}\right\|_{X}^{2}\right)+\frac{1}{2} \sum_{E \in \mathcal{E}_{h}} \int_{\partial E_{-}}\left|\left\{\boldsymbol{U}^{j}\right\} \cdot \boldsymbol{n}_{E}\right|\left\|\left[\tilde{\boldsymbol{U}}^{j+1}\right]\right\|^{2} \\
& +\frac{1}{2} \sum_{E \in \mathcal{E}_{h}} \int_{\partial E_{-} \cap(\partial \Omega)}\left|\boldsymbol{U}^{j} \cdot \boldsymbol{n}_{E}\right|\left\|\tilde{\boldsymbol{U}}^{j+1}\right\|^{2} \leq \frac{\epsilon}{2}\left\|\tilde{\boldsymbol{U}}^{j+1}\right\|_{X}^{2}+\frac{C_{0}^{2}}{2 \epsilon}\left\|\check{\boldsymbol{f}}^{j+1}\right\|_{L^{2}(\Omega)}^{2}
\end{aligned}
$$

Finally, (2.6) is obtained by choosing $\epsilon=\frac{\mu}{2}$ and summing over $j$.

Proposition 2.3 has a corollary similar to Corollary 2.2.

Remark 2.4. The estimate (2.6) is slightly better than (2.1) because it does not involve the factor $K$ that is likely to be smaller than one half. On the other hand, it does not give an estimate for $\sum_{j=1}^{N} \Delta t\left\|\boldsymbol{U}^{j}-\tilde{\boldsymbol{U}}^{j}\right\|_{X}^{2}$, but we shall not use this term further on.

\section{3. $L^{r}$ estimates}

In the sequel, we shall require some estimates in $L^{r}$ and interpolation estimates for the functions of $\boldsymbol{X}_{h}$. These are a refinement of the $L^{r}$ estimates proven in Lemma 6.2 of [14].

We first define a postprocessing technique: with any function $\boldsymbol{u}_{h}$ in $\boldsymbol{X}_{h}$, we want to associate a function $\overline{\boldsymbol{u}}_{h}$ in $\overline{\boldsymbol{X}}_{h}$ (see 1.23). To this end, given an interior edge $e \in \Gamma_{h}$ common to two elements $E_{e}^{1}$ and $E_{e}^{2}$ in $\mathcal{E}_{h}$, such that $\boldsymbol{n}_{e}$ is outward to $E_{e}^{1}$, we construct a piecewise $\mathbb{P}_{1}$ function $\lambda_{e}$ as follows. Let $\boldsymbol{b}_{e}$ denote the midpoint of $e$ and let $\lambda_{e} \in \mathbb{P}_{1}(E)$ for all $E \in \mathcal{E}_{h}$ be defined by

$$
\left.\lambda_{e}\left(\boldsymbol{b}_{e}\right)\right|_{E_{e}^{1}}=1,\left.\quad \lambda_{e}\left(\boldsymbol{b}_{e}\right)\right|_{E_{e}^{2}}=0, \quad \lambda_{e}\left(\boldsymbol{b}_{e^{\prime}}\right)=0 \quad \forall e^{\prime} \in \Gamma_{h}, \quad e^{\prime} \neq e .
$$

Thus, $\lambda_{e}$ vanishes over all triangles other than $E_{e}^{1}$ and

$$
\left[\lambda_{e}\right]\left(\boldsymbol{b}_{e}\right)=1, \quad \frac{1}{|e|} \int_{e}\left[\lambda_{e}\right]=1, \quad \int_{e^{\prime}}\left[\lambda_{e}\right]=0, \quad \forall e^{\prime} \in \Gamma_{h}, \quad e^{\prime} \neq e .
$$


If $e \in \Gamma_{h}$ lies on $\partial \Omega$ then we simply set

$$
\lambda_{e}\left(\boldsymbol{b}_{e}\right)=1, \quad \lambda_{e}\left(\boldsymbol{b}_{e^{\prime}}\right)=0, \quad \forall e^{\prime} \in \Gamma_{h}, \quad e^{\prime} \neq e .
$$

Now, for any $\boldsymbol{u}_{h} \in \boldsymbol{X}_{h}$, define $\overline{\boldsymbol{u}}_{h} \in \boldsymbol{X}_{h}$ by:

$$
\overline{\boldsymbol{u}}_{h}=\boldsymbol{u}_{h}-\sum_{e \in \Gamma_{h}}\left(\frac{1}{|e|} \int_{e}\left[\boldsymbol{u}_{h}\right]\right) \lambda_{e} .
$$

Then,

$$
\forall e \in \Gamma_{h}, \quad \int_{e}\left[\overline{\boldsymbol{u}}_{h}\right]=\int_{e}\left[\boldsymbol{u}_{h}\right]-\frac{1}{|e|} \int_{e}\left[\boldsymbol{u}_{h}\right] \int_{e}\left[\lambda_{e}\right]=\mathbf{0},
$$

and hence $\overline{\boldsymbol{u}}_{h} \in \overline{\boldsymbol{X}}_{h}$. The next two lemmas show that $\overline{\boldsymbol{u}}_{h}$ and $\boldsymbol{u}_{h}$ are closely related.

Lemma 3.1. There exists a constant $C$, independent of $h$, such that

$$
\forall \boldsymbol{u}_{h} \in \boldsymbol{X}_{h}, \quad\left\|\boldsymbol{u}_{h}-\overline{\boldsymbol{u}}_{h}\right\|_{X} \leq C J_{0}\left(\boldsymbol{u}_{h}, \boldsymbol{u}_{h}\right)^{1 / 2} .
$$

Proof. It suffices to consider a component of $\boldsymbol{u}_{h}$, denoted by $u_{h}$. Let us first study the gradient part of the norm. By (1.4), we have for any $E \in \mathcal{E}_{h}$ :

$$
\begin{aligned}
\left\|\nabla\left(u_{h}-\bar{u}_{h}\right)\right\|_{L^{2}(E)} & \leq \sum_{e \in \partial E} \frac{1}{|e|}\left|\int_{e}\left[u_{h}\right]\right|\left\|\nabla \lambda_{e}\right\|_{L^{2}(E)} \\
& \leq C_{1}|E|^{1 / 2} \frac{1}{\rho_{E}} \sum_{e \in \partial E} \frac{1}{|e|^{1 / 2}}\left\|\left[u_{h}\right]\right\|_{L^{2}(e)}\left\|\hat{\nabla} \hat{\lambda}_{e}\right\|_{L^{2}(\hat{E})} \leq C_{2} \sum_{e \in \partial E} \frac{1}{|e|^{1 / 2}}\left\|\left[u_{h}\right]\right\| \|_{L^{2}(e)}
\end{aligned}
$$

where here and in the sequel, the hat superscript denotes the reference element and quantities related to the reference element. Thus,

$$
\left(\sum_{E \in \mathcal{E}_{h}}\left\|\nabla\left(u_{h}-\bar{u}_{h}\right)\right\|_{L^{2}(E)}^{2}\right)^{1 / 2} \leq C_{3} J_{0}\left(u_{h}, u_{h}\right)^{1 / 2}
$$

Next, we consider the jump term. For any interior $e \in \Gamma_{h}$, the set of edges $e^{\prime} \in \Gamma_{h}$ for which $\left.\left[\lambda_{e^{\prime}}\right]\right|_{e} \neq 0$ is a subset of the union of $e$ and the edges of $\partial\left(E_{e}^{1} \cup E_{e}^{2}\right)$, and if $e \subset \partial \Omega \cap \partial E_{e}^{1}$, it is a subset of $\partial E_{e}^{1}$; we denote this set by $S_{e}$. Therefore,

$$
\left\|\left[u_{h}-\bar{u}_{h}\right]\right\|_{L^{2}(e)} \leq C_{4}|e|^{1 / 2} \sum_{e^{\prime} \in S_{e}} \frac{1}{\left|e^{\prime}\right|^{1 / 2}}\left\|\left[u_{h}\right]\right\|_{L^{2}\left(e^{\prime}\right)}\left\|\left[\hat{\lambda}_{e^{\prime}}\right]\right\|_{L^{2}(\hat{e})}
$$

Hence,

and thus

$$
\frac{1}{|e|^{1 / 2}}\left\|\left[u_{h}-\bar{u}_{h}\right]\right\|_{L^{2}(e)} \leq C_{5} \sum_{e^{\prime} \in S_{e}} \frac{1}{\left|e^{\prime}\right|^{1 / 2}}\left\|\left[u_{h}\right]\right\|_{L^{2}\left(e^{\prime}\right)}
$$

This completes the proof.

$$
J_{0}\left(u_{h}-\bar{u}_{h}, u_{h}-\bar{u}_{h}\right)^{1 / 2} \leq C_{6} J_{0}\left(u_{h}, u_{h}\right)^{1 / 2} .
$$

Lemma 3.2. For any $r \in[2, \infty]$, there exists a constant $C_{r}$ depending on $r$ but not on $h$, such that

$$
\forall \boldsymbol{u}_{h} \in \boldsymbol{X}_{h}, \quad\left\|\boldsymbol{u}_{h}-\overline{\boldsymbol{u}}_{h}\right\|_{L^{r}(\Omega)} \leq C_{r} h^{2 / r} J_{0}\left(\boldsymbol{u}_{h}, \boldsymbol{u}_{h}\right)^{1 / 2}
$$


Proof. Let $E \in \mathcal{E}_{h}$ and let $2 \leq r<\infty$. As in the proof of (3.3), we write for any component $u_{h}$ of $\boldsymbol{u}_{h}$ :

$$
\left\|u_{h}-\bar{u}_{h}\right\|_{L^{r}(E)} \leq C_{1}|E|^{1 / r} \sum_{e \in \partial E} \frac{1}{|e|^{1 / 2}}\left\|\left[u_{h}\right]\right\|_{L^{2}(e)} .
$$

Then summing over all $E$ and applying Jensen's inequality (that is valid since $r \geq 2$ ), we obtain

$$
\left\|u_{h}-\bar{u}_{h}\right\|_{L^{r}(\Omega)} \leq C_{2} h^{2 / r} J_{0}\left(u_{h}, u_{h}\right)^{1 / 2} .
$$

When $r=\infty$, let $E$ be any element where the maximum value of $\left|u_{h}-\bar{u}_{h}\right|$ is attained. Then,

$$
\max _{x \in E}\left|u_{h}(x)-\bar{u}_{h}(x)\right| \leq C_{3} \sum_{e \in \partial E} \frac{1}{|e|^{1 / 2}}\left\|\left[u_{h}\right]\right\|_{L^{2}(e)},
$$

and we recover again (3.4).

Lemma 3.3. There exists a constant $C$, independent of $h$, such that

$$
\forall \overline{\boldsymbol{u}}_{h} \in \overline{\boldsymbol{X}}_{h}, \quad J_{0}\left(\overline{\boldsymbol{u}}_{h}, \overline{\boldsymbol{u}}_{h}\right)^{1 / 2} \leq C\left\|\nabla \overline{\boldsymbol{u}}_{h}\right\|_{L^{2}(\Omega)} .
$$

Proof. Let $u_{h}$ be a component of $\overline{\boldsymbol{u}}_{h} \in \overline{\boldsymbol{X}}_{h}$. For any $e \in \Gamma_{h}$, as $\left.u_{h}\right|_{e}$ has the same mean value, denoted by $m_{e}$, coming from $E_{e}^{1}$ and from $E_{e}^{2}$, we can write (for simplicity, we assume that $|\hat{e}|=1$ ):

$$
\begin{aligned}
\left\|\left[u_{h}\right]\right\|_{L^{2}(e)} & =|e|^{1 / 2}\left\|\left.\hat{u}_{h}\right|_{\hat{E}^{1}}-\left.\hat{u}_{h}\right|_{\hat{E}^{2}}\right\|_{L^{2}(\hat{e})}=|e|^{1 / 2}\left\|\left.\left(\hat{u}_{h}-m_{e}\right)\right|_{\hat{E}^{1}}-\left.\left(\hat{u}_{h}-m_{e}\right)\right|_{\hat{E}^{2}}\right\|_{L^{2}(\hat{e})} \\
& \leq|e|^{1 / 2}\left(\left\|\left.\left(\hat{u}_{h}-m_{e}\right)\right|_{\hat{E}^{1}}\right\|_{L^{2}(\hat{e})}+\left\|\left.\left(\hat{u}_{h}-m_{e}\right)\right|_{\hat{E}^{2}}\right\|_{L^{2}(\hat{e})}\right) .
\end{aligned}
$$

Similarly, if $e$ lies on $\partial \Omega$, since the mean value $m_{e}=0$, we have

$$
\left\|\left[u_{h}\right]\right\|_{L^{2}(e)} \leq|e|^{1 / 2}\left\|\hat{u}_{h}-m_{e}\right\|_{L^{2}(\hat{e})} .
$$

As the mean-value is preserved by the transformation that maps $e$ onto $\hat{e}$, we obtain

$$
\left\|\left[u_{h}\right]\right\|_{L^{2}(e)} \leq C_{1}|e|^{1 / 2}\left(\left\|\hat{\nabla} \hat{u}_{h}\right\|_{L^{2}\left(\hat{E}^{1}\right)}+\left\|\hat{\nabla} \hat{u}_{h}\right\|_{L^{2}\left(\hat{E}^{2}\right)}\right) .
$$

Hence,

thus implying (3.5).

$$
\frac{1}{|e|^{1 / 2}}\left\|\left[u_{h}\right]\right\|_{L^{2}(e)} \leq C_{2}\left(\left\|\nabla u_{h}\right\|_{L^{2}\left(E_{e}^{1}\right)}+\left\|\nabla u_{h}\right\|_{L^{2}\left(E_{e}^{2}\right)}\right)
$$

Now we define a lifting function $\overline{\boldsymbol{u}}(h)$ of $\overline{\boldsymbol{u}}_{h}$, as in [14]: $\overline{\boldsymbol{u}}(h) \in\left(H_{0}^{1}(\Omega)\right)^{2}$ is the only solution of

$$
\forall \boldsymbol{v} \in\left(H_{0}^{1}(\Omega)\right)^{2}, \quad \int_{\Omega} \nabla \overline{\boldsymbol{u}}(h): \nabla \boldsymbol{v}=\sum_{E \in \mathcal{E}_{h}} \int_{E} \nabla \overline{\boldsymbol{u}}_{h}: \nabla \boldsymbol{v} .
$$

From (3.6) and (3.3), we immediately derive that

$$
\|\nabla \overline{\boldsymbol{u}}(h)\|_{L^{2}(\Omega)} \leq\left\|\nabla \overline{\boldsymbol{u}}_{h}\right\|_{L^{2}(\Omega)} \leq C\left\|\boldsymbol{u}_{h}\right\|_{X} .
$$

Lemma 3.4. For any $r \in[2, \infty)$, there exists a constant $C_{r}$, depending on $r$ but not on $h$, such that

$$
\begin{gathered}
\left\|\boldsymbol{u}_{h}-\overline{\boldsymbol{u}}(h)\right\|_{L^{r}(\Omega)} \leq C_{r} h^{2 / r} J_{0}\left(\boldsymbol{u}_{h}, \boldsymbol{u}_{h}\right)^{1 / 2} \quad \text { if } \quad r \geq 4, \\
\left\|\boldsymbol{u}_{h}-\overline{\boldsymbol{u}}(h)\right\|_{L^{r}(\Omega)} \leq C_{r} h^{1 / 2} J_{0}\left(\boldsymbol{u}_{h}, \boldsymbol{u}_{h}\right)^{1 / 2} \quad \text { if } \quad 2 \leq r<4 .
\end{gathered}
$$


Proof. Again, we consider one component $u_{h}$ of $\boldsymbol{u}_{h}$. Since we have (3.4), it suffices to prove that (3.8), (3.9) hold for $\bar{u}_{h}-\bar{u}(h)$. The proof is similar, but sharper than that of Lemma 6.2 of [14]. We proceed by duality and write with $1 / r^{\prime}+1 / r=1$ :

$$
\left\|\bar{u}_{h}-\bar{u}(h)\right\|_{L^{r}(\Omega)}=\sup _{g \in L^{r^{\prime}}(\Omega)} \frac{\int_{\Omega}\left(\bar{u}_{h}-\bar{u}(h)\right) g}{\|g\|_{L^{r^{\prime}}(\Omega)}} .
$$

For a fixed $g$ in $L^{r^{\prime}}(\Omega)$, let $\phi \in H_{0}^{1}(\Omega)$ solve:

$$
-\Delta \phi=g,\left.\quad \phi\right|_{\partial \Omega}=0
$$

When $r \geq 4$, then $r^{\prime} \leq 4 / 3$ and it follows from [16] that $\phi \in W^{2, r^{\prime}}(\Omega)$ with

$$
\|\phi\|_{W^{2, r^{\prime}}(\Omega)} \leq C_{1}(r)\|g\|_{L^{r^{\prime}(\Omega)}} .
$$

When $r<4$, then $r^{\prime}>4 / 3$ and $g$ belongs always to $L^{4 / 3}(\Omega)$. Therefore we also have $\phi \in W^{2,4 / 3}(\Omega)$ with

$$
\|\phi\|_{W^{2,4 / 3}(\Omega)} \leq C_{1}(4)\|g\|_{L^{4 / 3}(\Omega)} \leq C_{2}(r)\|g\|_{L^{r^{\prime}}(\Omega)}
$$

From (3.11), we derive

$$
\begin{aligned}
\int_{\Omega}\left(\bar{u}_{h}-\bar{u}(h)\right) g & =-\int_{\Omega} \Delta \phi\left(\bar{u}_{h}-\bar{u}(h)\right) \\
& =\sum_{E \in \mathcal{E}_{h}} \int_{E} \nabla \phi \cdot\left(\nabla \bar{u}_{h}-\nabla \bar{u}(h)\right)-\sum_{E \in \mathcal{E}_{h}} \int_{\partial E} \nabla \phi \cdot \boldsymbol{n}_{E}\left(\bar{u}_{h}-\bar{u}(h)\right)=-\sum_{e \in \Gamma_{h}} \int_{e} \nabla \phi \cdot \boldsymbol{n}_{e}\left[\bar{u}_{h}\right]
\end{aligned}
$$

owing to (3.6) and the regularity of $\phi$ and $\bar{u}(h)$. Thus, the zero mean-value of $\left[\bar{u}_{h}\right]$ on each $e$ implies that for any constants $c_{e}$, we have:

$$
\int_{\Omega}\left(\bar{u}_{h}-\bar{u}(h)\right) g=-\sum_{e \in \Gamma_{h}} \int_{e}\left(\nabla \phi \cdot \boldsymbol{n}_{e}-c_{e}\right)\left[\bar{u}_{h}\right] .
$$

Let $E$ be a triangle adjacent to $e$ and take $c_{e}=\boldsymbol{c} \cdot \boldsymbol{n}_{e}$, where

$$
\boldsymbol{c}=\frac{1}{|E|} \int_{E} \nabla \phi
$$

Let $4 \leq r<\infty$. Then $1<r^{\prime} \leq 4 / 3$ and the trace of $\nabla \phi$ on each edge $e$ belongs to $\left(L^{s^{\prime}}(e)\right)^{2}$ with $1 / s^{\prime}=2 / r^{\prime}-1$ and $1<s^{\prime} \leq 2$. Then, with $\frac{1}{s^{\prime}}+\frac{1}{s}=1$,

$$
\left|\int_{e}(\nabla \phi-\boldsymbol{c}) \cdot \boldsymbol{n}_{e}\left[\bar{u}_{h}\right]\right| \leq\|\nabla \phi-\boldsymbol{c}\|_{L^{s^{\prime}}(e)}\left\|\left[\bar{u}_{h}\right]\right\|_{L^{s}(e)} .
$$

On one hand, passing to the reference element, applying the trace theorem with this value of $s^{\prime}$ and using the definition of $\boldsymbol{c}$, we have

$$
\|\nabla \phi-c\|_{L^{s^{\prime}}(e)} \leq C_{3}|e|^{1 / s^{\prime}}|\widehat{\nabla \phi}|_{W^{1, r^{\prime}}(\hat{E})} \leq C_{4}|e|^{1 / s^{\prime}} h_{E}|E|^{-1 / r^{\prime}}|\nabla \phi|_{W^{1, r^{\prime}}(E)} \leq C_{5}|\nabla \phi|_{W^{1, r^{\prime}}(E)} .
$$

On the other hand, a local equivalence of norms gives:

$$
\left\|\left[\bar{u}_{h}\right]\right\|_{L^{s}(e)} \leq C_{6}|e|^{1 / s}\left\|\widehat{\left[\bar{u}_{h}\right]}\right\|_{L^{2}(\hat{e})} \leq C_{6}|e|^{1 / s-1 / 2}\left\|\left[\bar{u}_{h}\right]\right\|_{L^{2}(e)} .
$$


Combining these two inequalities, we obtain

$$
\left|\int_{e}(\nabla \phi-\boldsymbol{c}) \cdot \boldsymbol{n}_{e}\left[\bar{u}_{h}\right]\right| \leq C_{7} h^{2 / r}|\nabla \phi|_{W^{1, r^{\prime}}(E)} \frac{1}{|e|^{1 / 2}}\left\|\left[\bar{u}_{h}\right]\right\|_{L^{2}(e)} .
$$

Then, summing over $e$, applying Jensen's inequality and (3.12), we obtain for $r \geq 4$ :

$$
\left|\int_{\Omega}\left(\bar{u}_{h}-\bar{u}(h)\right) g\right| \leq C_{8} h^{2 / r} J_{0}\left(\bar{u}_{h}, \bar{u}_{h}\right)^{1 / 2}\|g\|_{L^{r^{\prime}}(\Omega)} .
$$

When $2 \leq r<4$, we apply the above result with the exponent $r^{\prime}=4 / 3$ and we use (3.13):

$$
\left|\int_{\Omega}\left(\bar{u}_{h}-\bar{u}(h)\right) g\right| \leq C_{9} h^{1 / 2} J_{0}\left(\bar{u}_{h}, \bar{u}_{h}\right)^{1 / 2}\|g\|_{L^{r^{\prime}}(\Omega)} .
$$

This concludes the proof.

Remark 3.5. Of course, by combining (3.7), (3.8) and (3.9) we recover the $L^{r}$ estimates of [14]: for any $r \in[2, \infty)$, there exists a constant $C_{r}$, depending on $r$ but not on $h$, such that

$$
\forall \boldsymbol{u}_{h} \in \boldsymbol{X}_{h}, \quad\left\|\boldsymbol{u}_{h}\right\|_{L^{r}(\Omega)} \leq C_{r}\left\|\boldsymbol{u}_{h}\right\|_{X}
$$

Remark 3.6. When $2 \leq r<4$, we can improve (3.9) by restricting the angles of $\partial \Omega$. In particular, if $r=2$ and $\Omega$ is convex, we recover a full power of $h$ :

$$
\left\|\boldsymbol{u}_{h}-\overline{\boldsymbol{u}}(h)\right\|_{L^{2}(\Omega)} \leq C h J_{0}\left(\boldsymbol{u}_{h}, \boldsymbol{u}_{h}\right)^{1 / 2}
$$

This follows from the fact that (3.13) is replaced by:

$$
\|\phi\|_{H^{2}(\Omega)} \leq C\|g\|_{L^{2}(\Omega)} .
$$

Remark 3.7. We easily derive from the above results that there exists a constant $C_{r}$, that depends on $r$ but not on $h$, such that for all $\boldsymbol{u}$ in $\left(H_{0}^{1}(\Omega)\right)^{2}$ and all $\boldsymbol{u}_{h}$ in $\boldsymbol{X}_{h}$, we have

$$
\left\|\boldsymbol{u}_{h}-\boldsymbol{u}\right\|_{L^{r}(\Omega)} \leq C_{r}\left\|\boldsymbol{u}_{h}-\boldsymbol{u}\right\|_{X}
$$

Indeed, since $\overline{\boldsymbol{u}}=\boldsymbol{u}$, we associate $\overline{\boldsymbol{u}}(h)$ to $\overline{\boldsymbol{u}}_{h}-\boldsymbol{u}$ as in (3.6). Hence, Sobolev's imbedding gives

$$
\left\|\boldsymbol{u}_{h}-\boldsymbol{u}\right\|_{L^{r}(\Omega)} \leq\left\|\boldsymbol{u}_{h}-\boldsymbol{u}-\overline{\boldsymbol{u}}(h)\right\|_{L^{r}(\Omega)}+C_{1}|\overline{\boldsymbol{u}}(h)|_{H^{1}(\Omega)}
$$

Then, we easily check that (3.4), (3.8) and (3.9) hold for $\boldsymbol{u}_{h}-\boldsymbol{u}$ and it suffices to bound $|\overline{\boldsymbol{u}}(h)|_{H^{1}(\Omega)}$. But

$$
|\overline{\boldsymbol{u}}(h)|_{H^{1}(\Omega)} \leq\left\|\nabla\left(\overline{\boldsymbol{u}}_{h}-\boldsymbol{u}\right)\right\|_{L^{2}(\Omega)} .
$$

Then Lemma 3.1 gives the result.

Finally, when $r=4$, we derive an analogue of the well-known interpolation inequality, that is valid in $H_{0}^{1}(\Omega)$ :

$$
\forall v \in H_{0}^{1}(\Omega), \quad\|v\|_{L^{4}(\Omega)} \leq 2^{1 / 4}\|v\|_{L^{2}(\Omega)}^{1 / 2}\|\nabla v\|_{L^{2}(\Omega)}^{1 / 2}
$$


Theorem 3.8. There exist constants $C_{i}, 1 \leq i \leq 3$, independent of $h$, such that

$$
\forall \boldsymbol{u}_{h} \in \boldsymbol{X}_{h}, \quad\left\|\boldsymbol{u}_{h}\right\|_{L^{4}(\Omega)} \leq C_{1}\left\|\boldsymbol{u}_{h}\right\|_{L^{2}(\Omega)}^{1 / 2}\left\|\boldsymbol{u}_{h}\right\|_{X}^{1 / 2}+C_{2} h^{1 / 4} J_{0}\left(\boldsymbol{u}_{h}, \boldsymbol{u}_{h}\right)^{1 / 4}\left\|\boldsymbol{u}_{h}\right\|_{X}^{1 / 2}+C_{3} h^{1 / 2} J_{0}\left(\boldsymbol{u}_{h}, \boldsymbol{u}_{h}\right)^{1 / 2}
$$

When $\Omega$ is convex, the above inequality can be improved

$$
\forall \boldsymbol{u}_{h} \in \boldsymbol{X}_{h}, \quad\left\|\boldsymbol{u}_{h}\right\|_{L^{4}(\Omega)} \leq C_{1}\left\|\boldsymbol{u}_{h}\right\|_{L^{2}(\Omega)}^{1 / 2}\left\|\boldsymbol{u}_{h}\right\|_{X}^{1 / 2}+C_{2} h^{1 / 2} J_{0}\left(\boldsymbol{u}_{h}, \boldsymbol{u}_{h}\right)^{1 / 4}\left\|\boldsymbol{u}_{h}\right\|_{X}^{1 / 2}+C_{3} h^{1 / 2} J_{0}\left(\boldsymbol{u}_{h}, \boldsymbol{u}_{h}\right)^{1 / 2}
$$

Proof. Consider one component $u_{h}$ of $\boldsymbol{u}_{h}$. From (3.8) and (3.16) we infer

$$
\left\|u_{h}\right\|_{L^{4}(\Omega)} \leq\left\|u_{h}-\bar{u}(h)\right\|_{L^{4}(\Omega)}+\|\bar{u}(h)\|_{L^{4}(\Omega)} \leq C_{1} h^{1 / 2} J_{0}\left(u_{h}, u_{h}\right)^{1 / 2}+2^{1 / 4}\|\bar{u}(h)\|_{L^{2}(\Omega)}^{1 / 2}\|\nabla \bar{u}(h)\|_{L^{2}(\Omega)}^{1 / 2} .
$$

Then (3.7) and (3.9) give

$$
\left\|u_{h}\right\|_{L^{4}(\Omega)} \leq C_{1} h^{1 / 2} J_{0}\left(u_{h}, u_{h}\right)^{1 / 2}+C_{2}\left\|u_{h}\right\|_{X}^{1 / 2}\left(\left\|u_{h}\right\|_{L^{2}(\Omega)}^{1 / 2}+C_{3} h^{1 / 4} J_{0}\left(u_{h}, u_{h}\right)^{1 / 4}\right) .
$$

This implies (3.17). When $\Omega$ is convex, the result follows by applying (3.15) instead of (3.9).

\section{First ERROR EStimate FOR VELOCITY}

In this section, we obtain a first error estimate for the velocity that is suboptimal in time, namely of the order $O\left(h+\Delta t^{1 / 2}\right)$. An improved optimal estimate is obtained in Section 5 .

We shall need the following estimates for the trilinar form $c$. The proof is similar to that of Lemma 6.4 of [14], but we write it here for the reader's convenience.

Proposition 4.1. (i) Assume that $\boldsymbol{u} \in W^{1, r}(\Omega)^{2}$ for some $r>2$. There exists a constant $C$ that is independent of $h$, such that for all $\boldsymbol{v}_{h} \in \boldsymbol{V}_{h}$ and $\boldsymbol{w}_{h} \in \boldsymbol{X}_{h}$,

$$
\left|c\left(\boldsymbol{v}_{h} ; \boldsymbol{u}, \boldsymbol{w}_{h}\right)\right| \leq C|\boldsymbol{u}|_{W^{1, r}(\Omega)}\left\|\boldsymbol{w}_{h}\right\|_{X}\left\|\boldsymbol{v}_{h}\right\|_{L^{2}(\Omega)} .
$$

(ii) If $\boldsymbol{u} \in H^{2}(\Omega)^{2}$, then for any $\boldsymbol{z} \in \boldsymbol{X}, \boldsymbol{v}_{h} \in \boldsymbol{V}_{h}$ and $\boldsymbol{w} \in \boldsymbol{X}_{h}$ we have

$$
\left|c^{\boldsymbol{z}}\left(\boldsymbol{v}_{h} ; R_{h} \boldsymbol{u}-\boldsymbol{u}, \boldsymbol{w}_{h}\right)\right| \leq C\left(\left|R_{h} \boldsymbol{u}-\boldsymbol{u}\right|_{W^{1, r}(\Omega)}+h^{1 / 2}|\boldsymbol{u}|_{H^{2}(\Omega)}\right)\left\|\boldsymbol{v}_{h}\right\|_{L^{2}(\Omega)}\left\|\boldsymbol{w}_{h}\right\|_{X}
$$

(iii) Finally, for any $\boldsymbol{z} \in \boldsymbol{X}, \boldsymbol{v}_{h}, \boldsymbol{u}_{h}$ and $\boldsymbol{w}_{h}$ in $\boldsymbol{X}_{h}$, we have

$$
\left|c^{\boldsymbol{z}}\left(\boldsymbol{v}_{h} ; \boldsymbol{u}_{h}, \boldsymbol{w}_{h}\right)\right| \leq C\left\|\boldsymbol{v}_{h}\right\|_{X}\left\|\boldsymbol{u}_{h}\right\|_{X}\left\|\boldsymbol{w}_{h}\right\|_{X} .
$$

Proof. (i) Since $\boldsymbol{u}$ has no jumps, we can write:

$$
c\left(\boldsymbol{v}_{h} ; \boldsymbol{u}, \boldsymbol{w}_{h}\right)=\sum_{E \in \mathcal{E}_{h}} \int_{E}\left(\boldsymbol{v}_{h} \cdot \nabla \boldsymbol{u}\right) \cdot \boldsymbol{w}_{h}-\frac{1}{2} b\left(\boldsymbol{v}_{h}, \boldsymbol{u} \cdot \boldsymbol{w}_{h}\right)
$$

The first term is bounded by virtue of (3.14):

$$
\sum_{E \in \mathcal{E}_{h}} \int_{E}\left(\boldsymbol{v}_{h} \cdot \nabla \boldsymbol{u}\right) \cdot \boldsymbol{w}_{h} \leq\left\|\boldsymbol{v}_{h}\right\|_{L^{2}(\Omega)}\|\nabla \boldsymbol{u}\|_{L^{r}(\Omega)}\left\|\boldsymbol{w}_{h}\right\|_{L^{r^{\prime}(\Omega)}} \leq C_{r^{\prime}}\left\|\boldsymbol{v}_{h}\right\|_{L^{2}(\Omega)}|\boldsymbol{u}|_{W^{1, r}(\Omega)}\left\|\boldsymbol{w}_{h}\right\|_{X}
$$


where $1 / r+1 / r^{\prime}=1 / 2, r>2, r^{\prime}>2$. To bound the second term, we use an argument of Girault and Lions [12]. Denote by $\boldsymbol{c}$ the piecewise constant that is, in each element $E$, the scalar product of two constant vectors $\boldsymbol{c}_{1} \cdot \boldsymbol{c}_{2}$. In view of $(1.14)$, we can write

$$
b\left(\boldsymbol{v}_{h}, \boldsymbol{u} \cdot \boldsymbol{w}_{h}\right)=b\left(\boldsymbol{v}_{h}, \boldsymbol{u} \cdot \boldsymbol{w}_{h}-\boldsymbol{c}_{1} \cdot \boldsymbol{c}_{2}\right)=b\left(\boldsymbol{v}_{h},\left(\boldsymbol{u}-\boldsymbol{c}_{1}\right) \cdot \boldsymbol{w}_{h}\right)+b\left(\boldsymbol{v}_{h}, \boldsymbol{c}_{1} \cdot\left(\boldsymbol{w}_{h}-\boldsymbol{c}_{2}\right)\right) .
$$

Let us choose in each $E$ :

From the definition of $r^{\prime}$, we have

$$
\boldsymbol{c}_{1}=\frac{1}{|E|} \int_{E} \boldsymbol{u}, \quad \boldsymbol{c}_{2}=\frac{1}{|E|} \int_{E} \boldsymbol{w}_{h}
$$

$$
\left\|\left(\boldsymbol{u}-\boldsymbol{c}_{1}\right) \cdot \boldsymbol{w}_{h}\right\|_{L^{2}(E)} \leq\left\|\boldsymbol{u}-\boldsymbol{c}_{1}\right\|_{L^{r}(E)}\left\|\boldsymbol{w}_{h}\right\|_{L^{r^{\prime}(E)}} \leq C_{1} h\left\|\boldsymbol{w}_{h}\right\|_{L^{r^{\prime}(E)}}|\boldsymbol{u}|_{W^{1, r}(E)}
$$

Similarly,

$$
\left\|\boldsymbol{c}_{1} \cdot\left(\boldsymbol{w}_{h}-\boldsymbol{c}_{2}\right)\right\|_{L^{2}(E)} \leq\left\|\boldsymbol{c}_{1}\right\|\left\|\boldsymbol{w}_{h}-\boldsymbol{c}_{2}\right\|_{L^{2}(E)} \leq C_{2} h\|\boldsymbol{u}\|_{L^{\infty}(E)}\left\|\nabla \boldsymbol{w}_{h}\right\|_{L^{2}(E)} .
$$

Hence, using locally an inverse inequality in each $E$, we have:

$$
\left|\int_{E} \nabla \cdot \boldsymbol{v}_{h}\left(\boldsymbol{u} \cdot \boldsymbol{w}_{h}-\boldsymbol{c}_{1} \cdot \boldsymbol{c}_{2}\right)\right| \leq C_{3}\left\|\boldsymbol{v}_{h}\right\|_{L^{2}(E)}\left(|\boldsymbol{u}|_{W^{1, r}(E)}\left\|\boldsymbol{w}_{h}\right\|_{L^{r^{\prime}(E)}}+\|\boldsymbol{u}\|_{L^{\infty}(E)}\left\|\nabla \boldsymbol{w}_{h}\right\|_{L^{2}(E)}\right) .
$$

To estimate the edge terms in $b$, we consider one element $E_{e}^{1}$ adjacent to $e$ and we apply the trace theorem:

$$
\left\|\left.\left(\boldsymbol{u}-\boldsymbol{c}_{1}\right) \cdot \boldsymbol{w}_{h}\right|_{E_{e}^{1}}\right\|_{L^{2}(e)} \leq C_{4}|e|^{1 / 2}\left(\left|E_{e}^{1}\right|^{-1 / 2}\left\|\left(\boldsymbol{u}-\boldsymbol{c}_{1}\right) \cdot \boldsymbol{w}_{h}\right\|_{L^{2}\left(E_{e}^{1}\right)}+\left\|\nabla\left(\left(\boldsymbol{u}-\boldsymbol{c}_{1}\right) \cdot \boldsymbol{w}_{h}\right)\right\|_{L^{2}\left(E_{e}^{1}\right)}\right) .
$$

We apply (4.4) to the first term and for the second term we write

$$
\left\|\nabla\left(\left(\boldsymbol{u}-\boldsymbol{c}_{1}\right) \cdot \boldsymbol{w}_{h}\right)\right\|_{L^{2}\left(E_{e}^{1}\right)} \leq\|\nabla \boldsymbol{u}\|_{L^{r}\left(E_{e}^{1}\right)}\left\|\boldsymbol{w}_{h}\right\|_{L^{r^{\prime}\left(E_{e}^{1}\right)}}+C_{5}\|\boldsymbol{u}\|_{L^{\infty}\left(E_{e}^{1}\right)}\left\|\nabla \boldsymbol{w}_{h}\right\|_{L^{2}\left(E_{e}^{1}\right)} .
$$

Hence, using a local equivalence of norms and denoting $E_{e}^{12}=E_{e}^{1} \cup E_{e}^{2}$, we obtain

$$
\left|\int_{e}\left\{\left(\boldsymbol{u}-\boldsymbol{c}_{1}\right) \cdot \boldsymbol{w}_{h}\right\}\left[\boldsymbol{v}_{h}\right] \cdot \boldsymbol{n}_{e}\right| \leq C_{6}\left\|\boldsymbol{v}_{h}\right\|_{L^{2}\left(E_{e}^{12}\right)}\left(|\boldsymbol{u}|_{W^{1, r}\left(E_{e}^{12}\right)}\left\|\boldsymbol{w}_{h}\right\|_{L^{r^{\prime}\left(E_{e}^{12}\right)}}+\|\boldsymbol{u}\|_{L^{\infty}\left(E_{e}^{12}\right)}\left\|\nabla \boldsymbol{w}_{h}\right\| L_{L^{2}\left(E_{e}^{12}\right)}\right) .
$$

The second edge term is easier since it only involves equivalent norms:

$$
\left|\int_{e}\left\{\boldsymbol{c}_{1} \cdot\left(\boldsymbol{w}_{h}-\boldsymbol{c}_{2}\right)\right\}\left[\boldsymbol{v}_{h}\right] \cdot \boldsymbol{n}_{e}\right| \leq C_{7}\|\boldsymbol{u}\|_{L^{\infty}\left(E_{e}^{12}\right)}\left\|\nabla \boldsymbol{w}_{h}\right\|_{L^{2}\left(E_{e}^{12}\right)}\left\|\boldsymbol{v}_{h}\right\|_{L^{2}\left(E_{e}^{12}\right)}
$$

Then summing over all elements and edges, applying Holder's inequality, (3.14) and Sobolev imbedding, we obtain:

$$
\forall \boldsymbol{v}_{h} \in \boldsymbol{V}_{h}, \quad \forall \boldsymbol{w}_{h} \in \boldsymbol{X}_{h}, \quad\left|b\left(\boldsymbol{v}_{h}, \boldsymbol{u} \cdot \boldsymbol{w}_{h}\right)\right| \leq C_{8}\left\|\boldsymbol{v}_{h}\right\|_{L^{2}(\Omega)}|\boldsymbol{u}|_{W^{1, r}(\Omega)}\left\|\boldsymbol{w}_{h}\right\|_{X}
$$

(ii) To establish (4.2), observe that the above argument applies to $R_{h} \boldsymbol{u}-\boldsymbol{u}$ instead of $\boldsymbol{u}$ for all except the upwind term. Using the approximation properties of $R_{h}$ and (3.14), the upwind term is bounded by

$$
\begin{aligned}
& C_{9} \sum_{e \in \Gamma_{h}}\left\|\boldsymbol{v}_{h}\right\|_{L^{4}(e)}\left\|\boldsymbol{w}_{h}\right\|_{L^{4}(e)}\left\|\left[R_{h} \boldsymbol{u}-\boldsymbol{u}\right]\right\|_{L^{2}(e)} \\
& \leq C_{10} \sum_{e \in \Gamma_{h}}|e|^{1 / 4}|E|^{-1 / 2}\left\|\boldsymbol{v}_{h}\right\|_{L^{2}(E)}|e|^{1 / 4}|E|^{-1 / 4}\left\|\boldsymbol{w}_{h}\right\|_{L^{4}(E)}|e|^{1 / 2}\left(|E|^{-1 / 2}\left\|R_{h} \boldsymbol{u}-\boldsymbol{u}\right\|_{L^{2}(E)}+\left\|\nabla\left(R_{h} \boldsymbol{u}-\boldsymbol{u}\right)\right\|_{L^{2}(E)}\right) \\
& \quad \leq C_{11} \sum_{E \in \mathcal{E}_{h}} h_{E}^{1 / 2}\left\|\boldsymbol{v}_{h}\right\|_{L^{2}(E)}\left\|\boldsymbol{w}_{h}\right\|_{L^{4}(E)}|\boldsymbol{u}|_{H^{2}(E)} \leq C_{12} h^{1 / 2}|\boldsymbol{u}|_{H^{2}(\Omega)}\left\|\boldsymbol{v}_{h}\right\|_{L^{2}(\Omega)}\left\|\boldsymbol{w}_{h}\right\| \|_{X}
\end{aligned}
$$


(iii) We skip the proof of (4.3) because it is straightforward. The proof of a sharper version is given in [14] when $\boldsymbol{v}_{h}$ belongs to $\boldsymbol{V}_{h}$.

We denote the errors between the numerical solutions and the approximation by $\boldsymbol{e}^{j}=\boldsymbol{U}^{j}-R_{h} \boldsymbol{u}^{j}$ and $\tilde{\boldsymbol{e}}^{j}=\tilde{\boldsymbol{U}}^{j}-R_{h} \boldsymbol{u}^{j}$, where $\boldsymbol{u}^{j}(\cdot)$ stands for $\boldsymbol{u}\left(t^{j}, \cdot\right)$. The following lemma shows exactly where the loss of optimality occurs. It is valid for both SIPG and NIPG.

Lemma 4.2. Assume that $p \in L^{2}\left(0, T ; H^{1}(\Omega)\right)$. Then for each $\eta>0$, there exists a constant $C$ that depends on $\eta$ but not on $h$, such that:

$$
\left|\int_{t^{j}}^{t^{j+1}} b\left(\tilde{\boldsymbol{e}}^{j+1}, p\right)\right| \leq \frac{1}{4}\left\|\tilde{\boldsymbol{e}}^{j+1}-\boldsymbol{e}^{j+1}\right\|_{L^{2}(\Omega)}^{2}+\eta \Delta t\left\|\boldsymbol{e}^{j+1}\right\|_{X}^{2}+\left(C h^{2}+\Delta t\right)\|\nabla p\|_{L^{2}\left(t^{j}, t^{j+1} ; L^{2}(\Omega)^{2}\right)}^{2} .
$$

Proof. Since $\boldsymbol{e}^{j+1} \in \boldsymbol{V}_{h}$, we can write

$$
b\left(\tilde{\boldsymbol{e}}^{j+1}, p\right)=b\left(\tilde{\boldsymbol{e}}^{j+1}-\boldsymbol{e}^{j+1}, p\right)+b\left(\boldsymbol{e}^{j+1}, p-r_{h} p\right) .
$$

Now, (1.13) implies

$$
\begin{array}{r}
\left|\int_{t^{j}}^{t^{j+1}} b\left(\tilde{\boldsymbol{e}}^{j+1}-\boldsymbol{e}^{j+1}, p\right)\right| \leq\left\|\tilde{\boldsymbol{e}}^{j+1}-\boldsymbol{e}^{j+1}\right\|_{L^{2}(\Omega)} \Delta t^{1 / 2}\|\nabla p\|_{L^{2}\left(t^{j}, t^{j+1} ; L^{2}(\Omega)^{2}\right)} \\
\quad \leq \frac{1}{4}\left\|\tilde{\boldsymbol{e}}^{j+1}-\boldsymbol{e}^{j+1}\right\|_{L^{2}(\Omega)}^{2}+\Delta t\|\nabla p\|_{L^{2}\left(t^{j}, t^{j+1} ; L^{2}(\Omega)^{2}\right)}^{2}
\end{array}
$$

On the other hand, the approximation property (1.35) of $r_{h}$ implies

$$
\left|b\left(\boldsymbol{e}^{j+1}, p-r_{h} p\right)\right| \leq C\left\|\boldsymbol{e}^{j+1}\right\|_{X} h\|\nabla p\|_{L^{2}(\Omega)} .
$$

Therefore, for any $\eta>0$,

$$
\left|\int_{t^{j}}^{t^{j+1}} b\left(\boldsymbol{e}^{j+1}, p-r_{h} p\right)\right| \leq \eta \Delta t\left\|\boldsymbol{e}^{j+1}\right\|_{X}^{2}+C h^{2}\|\nabla p\|_{L^{2}\left(t^{j}, t^{j+1} ; L^{2}(\Omega)^{2}\right)}^{2} .
$$

This concludes the proof of (4.6).

The next theorem establishes an a priori error estimate for SIPG.

Theorem 4.3. Assume that $\boldsymbol{u} \in L^{\infty}\left(0, T ; H^{2}(\Omega)^{2}\right), \boldsymbol{u}_{t} \in L^{2}\left(0, T ; H^{1}(\Omega)^{2}\right), p \in L^{2}\left(0, T ; H^{1}(\Omega)\right), \boldsymbol{u}_{0} \in \boldsymbol{V}$ and $\boldsymbol{U}^{0}=R_{h} \boldsymbol{u}_{0}$. If the ellipticy (1.22) holds, there exists a constant $C$, independent of $h$ and $\Delta t$, such that

$$
\begin{array}{r}
\max _{j}\left\|\boldsymbol{e}^{j}\right\|_{L^{2}(\Omega)}^{2}+\sum_{j=0}^{N-1}\left\|\tilde{\boldsymbol{e}}^{j+1}-\boldsymbol{e}^{j}\right\|_{L^{2}(\Omega)}^{2}+\frac{1}{2} \sum_{j=0}^{N-1}\left\|\tilde{\boldsymbol{e}}^{j+1}-\boldsymbol{e}^{j+1}\right\|_{L^{2}(\Omega)}^{2}+\sum_{j=0}^{N-1} \Delta t \sum_{E \in \mathcal{E}_{h}} \int_{\partial E_{-}}\left|\left\{\boldsymbol{U}^{j}\right\} \cdot \boldsymbol{n}_{E}\right|\left\|\left[\tilde{\boldsymbol{e}}^{j+1}\right]\right\|^{2} \\
+\sum_{j=0}^{N-1} \Delta t \sum_{E \in \mathcal{E}_{h}} \int_{\left(\partial E_{-}\right) \cap(\partial \Omega)}\left|\boldsymbol{U}^{j} \cdot \boldsymbol{n}_{E}\right|\left\|\tilde{\boldsymbol{e}}^{j+1}\right\|^{2}+\frac{1}{2} K \mu \sum_{j=0}^{N-1} \Delta t\left(\left\|\boldsymbol{e}^{j+1}\right\|_{X}^{2}+\left\|\tilde{\boldsymbol{e}}^{j+1}\right\|_{X}^{2}+\left\|\boldsymbol{e}^{j+1}-\tilde{\boldsymbol{e}}^{j+1}\right\|_{X}^{2}\right) \\
\leq C\left(h^{2}+\Delta t^{2}+\Delta t\right) .
\end{array}
$$


Proof. 1) Error equations

Integrating (1.26) between $t^{j}$ and $t^{j+1}$ and using (1.29), we derive:

$$
\begin{array}{r}
\forall \boldsymbol{v}_{h} \in \boldsymbol{X}_{h}, \quad\left(\tilde{\boldsymbol{U}}^{j+1}-\boldsymbol{U}^{j}, \boldsymbol{v}_{h}\right)+\mu \Delta t\left(a\left(\tilde{\boldsymbol{U}}^{j+1}, \boldsymbol{v}_{h}\right)+J_{0}\left(\tilde{\boldsymbol{U}}^{j+1}, \boldsymbol{v}_{h}\right)\right)+\int_{t^{j}}^{t^{j+1}} c^{j}\left(\boldsymbol{U}^{j} ; \tilde{\boldsymbol{U}}^{j+1}, \boldsymbol{v}_{h}\right) \\
=\left(\boldsymbol{u}^{j+1}-\boldsymbol{u}^{j}, \boldsymbol{v}_{h}\right)+\mu \int_{t^{j}}^{t^{j+1}}\left(a\left(\boldsymbol{u}, \boldsymbol{v}_{h}\right)+J_{0}\left(\boldsymbol{u}, \boldsymbol{v}_{h}\right)\right)+\int_{t^{j}}^{t^{j+1}} c\left(\boldsymbol{u} ; \boldsymbol{u}, \boldsymbol{v}_{h}\right)+\int_{t^{j}}^{t^{j+1}} b\left(\boldsymbol{v}_{h}, p\right) .
\end{array}
$$

Now inserting the approximations $R_{h} \boldsymbol{u}^{j+1}$ and $R_{h} \boldsymbol{u}^{j}$ and choosing $\boldsymbol{v}=\tilde{\boldsymbol{e}}^{j+1}$, we obtain a first error equation

$$
\begin{array}{r}
\left(\tilde{\boldsymbol{e}}^{j+1}-\boldsymbol{e}^{j}, \tilde{\boldsymbol{e}}^{j+1}\right)+\mu \Delta t\left(a\left(\tilde{\boldsymbol{e}}^{j+1}, \tilde{\boldsymbol{e}}^{j+1}\right)+J_{0}\left(\tilde{\boldsymbol{e}}^{j+1}, \tilde{\boldsymbol{e}}^{j+1}\right)\right)+\int_{t^{j}}^{t^{j+1}} c^{\boldsymbol{U}^{j}}\left(\boldsymbol{U}^{j} ; \tilde{\boldsymbol{e}}^{j+1}, \tilde{\boldsymbol{e}}^{j+1}\right)+\int_{t^{j}}^{t^{j+1}} c\left(\boldsymbol{e}^{j} ; \boldsymbol{u}, \tilde{\boldsymbol{e}}^{j+1}\right) \\
=\left(\boldsymbol{u}^{j+1}-R_{h} \boldsymbol{u}^{j+1}-\left(\boldsymbol{u}^{j}-R_{h} \boldsymbol{u}^{j}\right), \tilde{\boldsymbol{e}}^{j+1}\right)+\mu \int_{t^{j}}^{t^{j+1}}\left(a\left(\boldsymbol{u}-R_{h} \boldsymbol{u}^{j+1}, \tilde{\boldsymbol{e}}^{j+1}\right)+J_{0}\left(\boldsymbol{u}-R_{h} \boldsymbol{u}^{j+1}, \tilde{\boldsymbol{e}}^{j+1}\right)\right) \\
+\int_{t^{j}}^{t^{j+1}} c^{\boldsymbol{U}^{j}}\left(\boldsymbol{U}^{j} ; \boldsymbol{u}-R_{h} \boldsymbol{u}^{j+1}, \tilde{\boldsymbol{e}}^{j+1}\right)+\int_{t^{j}}^{t^{j+1}} c\left(\boldsymbol{u}-R_{h} \boldsymbol{u}^{j} ; \boldsymbol{u}, \tilde{\boldsymbol{e}}^{j+1}\right)+\int_{t^{j}}^{t^{j+1}} b\left(\tilde{\boldsymbol{e}}^{j+1}, p\right) .
\end{array}
$$

Applying (1.18), this implies

$$
\begin{aligned}
& \frac{1}{2}\left(\left\|\tilde{\boldsymbol{e}}^{j+1}\right\|_{L^{2}(\Omega)}^{2}-\right.\left.\left\|\boldsymbol{e}^{j}\right\|_{L^{2}(\Omega)}^{2}+\left\|\tilde{\boldsymbol{e}}^{j+1}-\boldsymbol{e}^{j}\right\|_{L^{2}(\Omega)}^{2}\right)+\mu \Delta t\left(a\left(\tilde{\boldsymbol{e}}^{j+1}, \tilde{\boldsymbol{e}}^{j+1}\right)+J_{0}\left(\tilde{\boldsymbol{e}}^{j+1}, \tilde{\boldsymbol{e}}^{j+1}\right)\right) \\
&+\frac{1}{2} \int_{t^{j}}^{t^{j+1}} \sum_{E \in \mathcal{E}_{h}} \int_{\partial E_{-}}\left|\left\{\boldsymbol{U}^{j}\right\} \cdot \boldsymbol{n}_{E}\right|\left\|\left[\tilde{\boldsymbol{e}}^{j+1}\right]\right\|^{2}+\frac{1}{2} \int_{t^{j}}^{t^{j+1}} \sum_{E \in \mathcal{E}_{h}} \int_{\left(\partial E_{-}\right) \cap(\partial \Omega)}\left|\boldsymbol{U}^{j} \cdot \boldsymbol{n}_{E}\right|\left\|\tilde{\boldsymbol{e}}^{j+1}\right\|^{2}+\int_{t^{j}}^{t^{j+1}} c\left(\boldsymbol{e}^{j} ; \boldsymbol{u}, \tilde{\boldsymbol{e}}^{j+1}\right) \\
&=\int_{t^{j}}^{t^{j+1}}\left(\left(\boldsymbol{u}-R_{h} \boldsymbol{u}\right)_{t}, \tilde{\boldsymbol{e}}^{j+1}\right)+\mu \int_{t^{j}}^{t^{j+1}}\left(a\left(\boldsymbol{u}-R_{h} \boldsymbol{u}^{j+1}, \tilde{\boldsymbol{e}}^{j+1}\right)+J_{0}\left(\boldsymbol{u}-R_{h} \boldsymbol{u}^{j+1}, \tilde{\boldsymbol{e}}^{j+1}\right)\right) \\
& \quad+\int_{t^{j}}^{t^{j+1}} c^{\boldsymbol{U}^{j}}\left(\boldsymbol{U}^{j} ; \boldsymbol{u}-R_{h} \boldsymbol{u}^{j+1}, \tilde{\boldsymbol{e}}^{j+1}\right)+\int_{t^{j}}^{t^{j+1}} c\left(\boldsymbol{u}-R_{h} \boldsymbol{u}^{j} ; \boldsymbol{u}, \tilde{\boldsymbol{e}}^{j+1}\right)+\int_{t^{j}}^{t^{j+1}} b\left(\tilde{\boldsymbol{e}}^{j+1}, p\right) . \quad(4.8)
\end{aligned}
$$

Similarly, inserting $R_{h} \boldsymbol{u}^{j+1}$ in (1.27), we get a second error equation:

$$
\forall \boldsymbol{v}_{h} \in \boldsymbol{X}_{h}, \quad \frac{1}{\Delta t}\left(\boldsymbol{e}^{j+1}-\tilde{\boldsymbol{e}}^{j+1}, \boldsymbol{v}_{h}\right)+\mu\left(a\left(\boldsymbol{e}^{j+1}-\tilde{\boldsymbol{e}}^{j+1}, \boldsymbol{v}_{h}\right)+J_{0}\left(\boldsymbol{e}^{j+1}-\tilde{\boldsymbol{e}}^{j+1}, \boldsymbol{v}_{h}\right)\right)+b\left(\boldsymbol{v}_{h}, P^{j+1}\right)=0
$$

Choosing $\boldsymbol{v}_{h}=e^{j+1}$, integrating between $t^{j}$ and $t^{j+1}$ and using (1.28) and the symmetry of $a$, we derive

$$
\begin{aligned}
& \frac{1}{2}\left(\left\|\boldsymbol{e}^{j+1}\right\|_{L^{2}(\Omega)}^{2}-\left\|\tilde{\boldsymbol{e}}^{j+1}\right\|_{L^{2}(\Omega)}^{2}+\left\|\boldsymbol{e}^{j+1}-\tilde{\boldsymbol{e}}^{j+1}\right\|_{L^{2}(\Omega)}^{2}\right)+\frac{\mu \Delta t}{2}\left(a\left(\boldsymbol{e}^{j+1}, \boldsymbol{e}^{j+1}\right)+J_{0}\left(\boldsymbol{e}^{j+1}, \boldsymbol{e}^{j+1}\right)\right. \\
& \left.\quad-a\left(\tilde{\boldsymbol{e}}^{j+1}, \tilde{\boldsymbol{e}}^{j+1}\right)-J_{0}\left(\tilde{\boldsymbol{e}}^{j+1}, \tilde{\boldsymbol{e}}^{j+1}\right)+a\left(\boldsymbol{e}^{j+1}-\tilde{\boldsymbol{e}}^{j+1}, \boldsymbol{e}^{j+1}-\tilde{\boldsymbol{e}}^{j+1}\right)+J_{0}\left(\boldsymbol{e}^{j+1}-\tilde{\boldsymbol{e}}^{j+1}, \boldsymbol{e}^{j+1}-\tilde{\boldsymbol{e}}^{j+1}\right)\right)=0 .
\end{aligned}
$$


Summing (4.8) and (4.9), we obtain a third error equation:

$$
\begin{aligned}
& \left\|\boldsymbol{e}^{j+1}\right\|_{L^{2}(\Omega)}^{2}-\left\|\boldsymbol{e}^{j}\right\|_{L^{2}(\Omega)}^{2}+\left\|\tilde{\boldsymbol{e}}^{j+1}-\boldsymbol{e}^{j}\right\|_{L^{2}(\Omega)}^{2}+\left\|\boldsymbol{e}^{j+1}-\tilde{\boldsymbol{e}}^{j+1}\right\|_{L^{2}(\Omega)}^{2}+\mu \Delta t\left(a\left(\boldsymbol{e}^{j+1}, \boldsymbol{e}^{j+1}\right)+J_{0}\left(\boldsymbol{e}^{j+1}, \boldsymbol{e}^{j+1}\right)\right. \\
& \left.+a\left(\tilde{\boldsymbol{e}}^{j+1}, \tilde{\boldsymbol{e}}^{j+1}\right)+J_{0}\left(\tilde{\boldsymbol{e}}^{j+1}, \tilde{\boldsymbol{e}}^{j+1}\right)+a\left(\boldsymbol{e}^{j+1}-\tilde{\boldsymbol{e}}^{j+1}, \boldsymbol{e}^{j+1}-\tilde{\boldsymbol{e}}^{j+1}\right)+J_{0}\left(\boldsymbol{e}^{j+1}-\tilde{\boldsymbol{e}}^{j+1}, \boldsymbol{e}^{j+1}-\tilde{\boldsymbol{e}}^{j+1}\right)\right) \\
& +\Delta t \sum_{E \in \mathcal{E}_{h}} \int_{\partial E_{-}}\left|\left\{\boldsymbol{U}^{j}\right\} \cdot \boldsymbol{n}_{E}\right|\left\|\left[\tilde{\boldsymbol{e}}^{j+1}\right]\right\|^{2}+\Delta t \sum_{E \in \mathcal{E}_{h}} \int_{\left(\partial E_{-}\right) \cap(\partial \Omega)}\left|\boldsymbol{U}^{j} \cdot \boldsymbol{n}_{E}\right|\left\|\tilde{\boldsymbol{e}}^{j+1}\right\|^{2}+2 \int_{t^{j}}^{t^{j+1}} c\left(\boldsymbol{e}^{j} ; \boldsymbol{u}, \tilde{\boldsymbol{e}}^{j+1}\right) \\
& =2 \int_{t^{j}}^{t^{j+1}}\left(\left(\boldsymbol{u}-R_{h} \boldsymbol{u}\right)_{t}, \tilde{\boldsymbol{e}}^{j+1}\right)+2 \mu \int_{t^{j}}^{t^{j+1}}\left(a\left(\boldsymbol{u}-R_{h} \boldsymbol{u}^{j+1}, \tilde{\boldsymbol{e}}^{j+1}\right)+J_{0}\left(\boldsymbol{u}-R_{h} \boldsymbol{u}^{j+1}, \tilde{\boldsymbol{e}}^{j+1}\right)\right) \\
& +2 \int_{t^{j}}^{t^{j+1}} c^{\boldsymbol{U}^{j}}\left(\boldsymbol{U}^{j} ; \boldsymbol{u}-R_{h} \boldsymbol{u}^{j+1}, \tilde{\boldsymbol{e}}^{j+1}\right)+2 \int_{t^{j}}^{t^{j+1}} c\left(\boldsymbol{u}-R_{h} \boldsymbol{u}^{j} ; \boldsymbol{u}, \tilde{\boldsymbol{e}}^{j+1}\right)+2 \int_{t^{j}}^{t^{j+1}} b\left(\tilde{\boldsymbol{e}}^{j+1}, p\right) .
\end{aligned}
$$

By virtue of (4.1), for any $\epsilon>0$, we have

$$
\left|2 \int_{t^{j}}^{t^{j+1}} c\left(\boldsymbol{e}^{j} ; \boldsymbol{u}, \tilde{\boldsymbol{e}}^{j+1}\right)\right| \leq \epsilon \Delta t\left\|\tilde{\boldsymbol{e}}^{j+1}\right\|_{X}^{2}+C\|\boldsymbol{u}\|_{L^{\infty}\left(0, T, W^{1, r}(\Omega)^{2}\right)}^{2} \Delta t\left\|\boldsymbol{e}^{j}\right\|_{L^{2}(\Omega)}^{2}
$$

in this proof, $C$ denotes various constants that depend on $\epsilon$, but not on $h$ and $\Delta t$. Therefore, from (1.22), the left-hand side of (4.10) is bounded below by

$$
\begin{array}{r}
\left\|\boldsymbol{e}^{j+1}\right\|_{L^{2}(\Omega)}^{2}-\left\|\boldsymbol{e}^{j}\right\|_{L^{2}(\Omega)}^{2}+\left\|\tilde{\boldsymbol{e}}^{j+1}-\boldsymbol{e}^{j}\right\|_{L^{2}(\Omega)}^{2}+\left\|\boldsymbol{e}^{j+1}-\tilde{\boldsymbol{e}}^{j+1}\right\|_{L^{2}(\Omega)}^{2}+\Delta t \sum_{E \in \mathcal{E}_{h}} \int_{\partial E_{-}}\left|\left\{\boldsymbol{U}^{j}\right\} \cdot \boldsymbol{n}_{E}\right|\left\|\left[\tilde{\boldsymbol{e}}^{j+1}\right]\right\|^{2} \\
+\Delta t \sum_{E \in \mathcal{E}_{h}} \int_{\left(\partial E_{-}\right) \cap(\partial \Omega)}\left|\boldsymbol{U}^{j} \cdot \boldsymbol{n}_{E}\right|\left\|\tilde{\boldsymbol{e}}^{j+1}\right\|^{2}+K \mu \Delta t\left(\left\|\boldsymbol{e}^{j+1}\right\|_{X}^{2}+\left\|\tilde{\boldsymbol{e}}^{j+1}\right\|_{X}^{2}+\left\|\boldsymbol{e}^{j+1}-\tilde{\boldsymbol{e}}^{j+1}\right\|_{X}^{2}\right) \\
-\epsilon \Delta t\left\|\tilde{\boldsymbol{e}}^{j+1}\right\|_{X}^{2}-C \Delta t\left\|\boldsymbol{e}^{j}\right\|_{L^{2}(\Omega)}^{2}\|\boldsymbol{u}\|_{L^{\infty}\left(0, T, W^{1, r}(\Omega)^{2}\right)^{2}}^{2}
\end{array}
$$

2) Upper bound of linear terms

We now bound the linear terms in the right-hand side of (4.10); first (1.34) gives

$$
\begin{aligned}
& \left|\int_{t^{j}}^{t^{j+1}}\left(\left(\boldsymbol{u}-R_{h} \boldsymbol{u}\right)_{t}, \tilde{\boldsymbol{e}}^{j+1}\right)\right| \leq C h\left\|\tilde{\boldsymbol{e}}^{j+1}\right\|_{L^{2}(\Omega)} \int_{t^{j}}^{t^{j+1}}\left\|\nabla \boldsymbol{u}_{t}\right\|_{L^{2}(\Omega)} \\
& \quad \leq C h \Delta t^{1 / 2}\left\|\tilde{\boldsymbol{e}}^{j+1}\right\|_{L^{2}(\Omega)}\left\|\boldsymbol{u}_{t}\right\|_{L^{2}\left(t^{j}, t^{j+1}, H^{1}(\Omega)^{2}\right)} \leq \epsilon \Delta t\left\|\tilde{\boldsymbol{e}}^{j+1}\right\|_{L^{2}(\Omega)}^{2}+C h^{2}\left\|\boldsymbol{u}_{t}\right\|_{L^{2}\left(t^{j}, t^{j+1}, H^{1}(\Omega)^{2}\right)}^{2} \\
& \quad
\end{aligned}
$$

We rewrite the second term as follows:

$$
a\left(R_{h} \boldsymbol{u}^{j+1}-\boldsymbol{u}, \tilde{\boldsymbol{e}}^{j+1}\right)=a\left(R_{h}\left(\boldsymbol{u}^{j+1}-\boldsymbol{u}\right), \tilde{\boldsymbol{e}}^{j+1}\right)+a\left(R_{h} \boldsymbol{u}-\boldsymbol{u}, \tilde{\boldsymbol{e}}^{j+1}\right) .
$$

Applying (1.20), (1.21) and (1.36) to the first term, it is bounded by

$$
\begin{aligned}
a\left(R_{h}\left(\boldsymbol{u}^{j+1}-\boldsymbol{u}\right), \tilde{\boldsymbol{e}}^{j+1}\right) & \leq C\left\|R_{h}\left(\boldsymbol{u}^{j+1}-\boldsymbol{u}\right)\right\|_{X}\left\|\tilde{\boldsymbol{e}}^{j+1}\right\|_{X} \leq C\left|\boldsymbol{u}^{j+1}-\boldsymbol{u}\right|_{H^{1}(\Omega)}\left\|\tilde{\boldsymbol{e}}^{j+1}\right\|_{X} \\
& \leq C\left(t^{j+1}-t\right)^{1 / 2}\left\|\boldsymbol{u}_{t}\right\|_{L^{2}\left(t^{j}, t^{j+1} ; H^{1}(\Omega)^{2}\right)}\left\|\tilde{\boldsymbol{e}}^{j+1}\right\|_{X} .
\end{aligned}
$$

Next, by Lemma 1.4,

$$
\left|a\left(R_{h} \boldsymbol{u}-\boldsymbol{u}, \tilde{\boldsymbol{e}}^{j+1}\right)\right| \leq C h|\boldsymbol{u}|_{H^{2}(\Omega)}\left\|\tilde{\boldsymbol{e}}^{j+1}\right\|_{X}
$$


Thus,

$$
\left|\int_{t^{j}}^{t^{j+1}} a\left(R_{h} \boldsymbol{u}^{j+1}-\boldsymbol{u}, \tilde{\boldsymbol{e}}^{j+1}\right)\right| \leq \epsilon \Delta t\left\|\tilde{\boldsymbol{e}}^{j+1}\right\|_{X}^{2}+C \Delta t^{2}\left\|\boldsymbol{u}_{t}\right\|_{L^{2}\left(t^{j}, t^{j+1}, H^{1}(\Omega)^{2}\right)}^{2}+C h^{2}\|\boldsymbol{u}\|_{L^{2}\left(t^{j}, t^{j+1}, H^{2}(\Omega)^{2}\right)}^{2} .
$$

Because of the regularity of $\boldsymbol{u}$, the jump term satisfies:

$$
J_{0}\left(R_{h} \boldsymbol{u}^{j+1}-\boldsymbol{u}, \tilde{\boldsymbol{e}}^{j+1}\right)=J_{0}\left(R_{h} \boldsymbol{u}^{j+1}-\boldsymbol{u}^{j+1}, \tilde{\boldsymbol{e}}^{j+1}\right) .
$$

Hence, by (1.38)

$$
\left|\int_{t^{j}}^{t^{j+1}} J_{0}\left(R_{h} \boldsymbol{u}^{j+1}-\boldsymbol{u}, \tilde{\boldsymbol{e}}^{j+1}\right)\right| \leq C h \Delta t\left|\boldsymbol{u}^{j+1}\right|_{H^{2}(\Omega)}\left\|\tilde{\boldsymbol{e}}^{j+1}\right\|_{X} \leq \epsilon \Delta t\left\|\tilde{\boldsymbol{e}}^{j+1}\right\|_{X}^{2}+C h^{2} \Delta t\|\boldsymbol{u}\|_{L^{\infty}\left(0, T ; H^{2}(\Omega)^{2}\right)^{2}}^{2} .
$$

3) Upper bound of nonlinear terms

Now, we estimate the nonlinear terms. The first nonlinear term is split as follows:

$$
\begin{aligned}
{ }_{c}^{\boldsymbol{U}^{j}}\left(\boldsymbol{U}^{j} ; R_{h} \boldsymbol{u}^{j+1}-\boldsymbol{u}, \tilde{\boldsymbol{e}}^{j+1}\right)= & c \boldsymbol{U}^{j}\left(\boldsymbol{e}^{j} ; R_{h} \boldsymbol{u}^{j+1}-\boldsymbol{u}, \tilde{\boldsymbol{e}}^{j+1}\right)+c^{\boldsymbol{U}^{j}}\left(R_{h} \boldsymbol{u}^{j} ; R_{h}\left(\boldsymbol{u}^{j+1}-\boldsymbol{u}\right), \tilde{\boldsymbol{e}}^{j+1}\right) \\
& +c^{\boldsymbol{U}^{j}}\left(R_{h} \boldsymbol{u}^{j} ; R_{h} \boldsymbol{u}-\boldsymbol{u}, \tilde{\boldsymbol{e}}^{j+1}\right) .
\end{aligned}
$$

By noting that the upwind term involving $R_{h} \boldsymbol{u}^{j+1}-\boldsymbol{u}$ is the same as the one involving $R_{h} \boldsymbol{u}^{j+1}-\boldsymbol{u}^{j+1}$, we can apply Proposition 4.1 to the first term in (4.15) and obtain

$$
\begin{aligned}
\left|c \boldsymbol{U}^{j}\left(\boldsymbol{e}^{j} ; R_{h} \boldsymbol{u}^{j+1}-\boldsymbol{u}, \tilde{\boldsymbol{e}}^{j+1}\right)\right| & \leq C\left\|\boldsymbol{e}^{j}\right\|_{L^{2}(\Omega)}\left\|\tilde{\boldsymbol{e}}^{j+1}\right\|_{X}\left(\left|R_{h} \boldsymbol{u}^{j+1}-\boldsymbol{u}\right|_{W^{1, r}(\Omega)}+h^{1 / 2}|\boldsymbol{u}|_{H^{2}(\Omega)}\right) \\
& \leq C\|\boldsymbol{u}\|_{L^{\infty}\left(0, T ; H^{2}(\Omega)^{2}\right)}\left\|\boldsymbol{e}^{j}\right\|_{L^{2}(\Omega)}\left\|\tilde{\boldsymbol{e}}^{j+1}\right\|_{X} .
\end{aligned}
$$

For the second term, by applying (4.3) and (1.36):

$$
\left|c \boldsymbol{U}^{j}\left(R_{h} \boldsymbol{u}^{j} ; R_{h}\left(\boldsymbol{u}^{j+1}-\boldsymbol{u}\right), \tilde{\boldsymbol{e}}^{j+1}\right)\right| \leq C\left(t^{j+1}-t\right)^{1 / 2}\left|\boldsymbol{u}^{j}\right|_{H^{1}(\Omega)}\left\|\boldsymbol{u}_{t}\right\|_{L^{2}\left(t^{j}, t^{j+1} ; H^{1}(\Omega)^{2}\right)}\left\|\tilde{\boldsymbol{e}}^{j+1}\right\|_{X} .
$$

The third term is bounded straightforwardly as follows:

$$
\left|c^{\boldsymbol{U}^{j}}\left(R_{h} \boldsymbol{u}^{j} ; R_{h} \boldsymbol{u}-\boldsymbol{u}, \tilde{\boldsymbol{e}}^{j+1}\right)\right| \leq C h\left|\boldsymbol{u}^{j}\right|_{H^{1}(\Omega)}\|\boldsymbol{u}\|_{H^{2}(\Omega)}\left\|\tilde{\boldsymbol{e}}^{j+1}\right\|_{X} .
$$

Thus, combining all terms in (4.15):

$$
\begin{aligned}
\left|\int_{t^{j}}^{t^{j+1}} c^{\boldsymbol{U}^{j}}\left(\boldsymbol{U}^{j} ; R_{h} \boldsymbol{u}^{j+1}-\boldsymbol{u}, \tilde{\boldsymbol{e}}^{j+1}\right)\right| \leq \epsilon \Delta t\left\|\tilde{\boldsymbol{e}}^{j+1}\right\|_{X}^{2}+C \Delta t\|\boldsymbol{u}\|_{L^{\infty}\left(0, T ; H^{2}(\Omega)^{2}\right)}^{2}\left\|\boldsymbol{e}^{j}\right\|_{L^{2}(\Omega)}^{2} \\
+C \Delta t^{2}\|\boldsymbol{u}\|_{L^{\infty}\left(0, T ; H^{1}(\Omega)^{2}\right)}^{2}\left\|\boldsymbol{u}_{t}\right\|_{L^{2}\left(t^{j}, t^{j+1} ; H^{1}(\Omega)^{2}\right)}^{2}+C h^{2}\|\boldsymbol{u}\|_{L^{\infty}\left(0, T ; H^{1}(\Omega)^{2}\right)}^{2}\|\boldsymbol{u}\|_{L^{2}\left(t^{j}, t^{j+1} ; H^{2}(\Omega)^{2}\right)}^{2}
\end{aligned}
$$

The other nonlinear term is rewritten as:

$$
\int_{t^{j}}^{t^{j+1}} c\left(R_{h} \boldsymbol{u}^{j}-\boldsymbol{u} ; \boldsymbol{u}, \tilde{\boldsymbol{e}}^{j+1}\right)=\int_{t^{j}}^{t^{j+1}} c\left(R_{h} \boldsymbol{u}^{j}-\boldsymbol{u}^{j} ; \boldsymbol{u}, \tilde{\boldsymbol{e}}^{j+1}\right)+\int_{t^{j}}^{t^{j+1}} c\left(\boldsymbol{u}^{j}-\boldsymbol{u} ; \boldsymbol{u}, \tilde{\boldsymbol{e}}^{j+1}\right) .
$$

A slight variant of the argument in Proposition 4.1 gives

$$
\left|c\left(R_{h} \boldsymbol{u}^{j}-\boldsymbol{u}^{j} ; \boldsymbol{u}, \tilde{\boldsymbol{e}}^{j+1}\right)\right| \leq C h^{2}\left|\boldsymbol{u}^{j}\right|_{H^{2}(\Omega)}|\boldsymbol{u}|_{W^{1,4}(\Omega)}\left\|\tilde{\boldsymbol{e}}^{j+1}\right\|_{X} .
$$


The second term reduces to:

$$
\begin{aligned}
c\left(\boldsymbol{u}^{j}-\boldsymbol{u} ; \boldsymbol{u}, \tilde{\boldsymbol{e}}^{j+1}\right) & =\sum_{E \in \mathcal{E}_{h}} \int_{E}\left(\boldsymbol{u}^{j}-\boldsymbol{u}\right) \cdot \nabla \boldsymbol{u} \cdot \tilde{\boldsymbol{e}}^{j+1}=-\int_{t^{j}}^{t} \sum_{E \in \mathcal{E}_{h}} \int_{E} \frac{\partial \boldsymbol{u}}{\partial \tau} \cdot \nabla \boldsymbol{u} \cdot \tilde{\boldsymbol{e}}^{j+1} \\
& \leq \int_{t^{j}}^{t}\left\|\frac{\partial \boldsymbol{u}}{\partial \tau}\right\|_{L^{2}(\Omega)}|\boldsymbol{u}|_{W^{1,4}(\Omega)}\left\|\tilde{\boldsymbol{e}}^{j+1}\right\|_{L^{4}(\Omega)} .
\end{aligned}
$$

Thus,

$$
\begin{gathered}
\left|\int_{t^{j}}^{t^{j+1}} c\left(R_{h} \boldsymbol{u}^{j}-\boldsymbol{u} ; \boldsymbol{u}, \tilde{\boldsymbol{e}}^{j+1}\right)\right| \leq C \Delta t^{1 / 2}\left\|\tilde{\boldsymbol{e}}^{j+1}\right\|_{X}\left(\Delta t\|\boldsymbol{u}\|_{L^{\infty}\left(0, T ; W^{1,4}(\Omega)^{2}\right)}\left\|\boldsymbol{u}_{t}\right\|_{L^{2}\left(t^{j}, t^{j+1} ; L^{2}(\Omega)^{2}\right)}\right. \\
\left.+C h^{2}\left|\boldsymbol{u}^{j}\right|_{H^{2}(\Omega)}\|\boldsymbol{u}\|_{L^{2}\left(t^{j}, t^{j+1} ; W^{1,4}(\Omega)^{2}\right)}\right) \\
\leq \epsilon \Delta t\left\|\tilde{\boldsymbol{e}}^{j+1}\right\|_{X}^{2}+C \Delta t^{2}\|\boldsymbol{u}\|_{L^{\infty}\left(0, T ; W^{1,4}(\Omega)^{2}\right)}^{2}\left\|\boldsymbol{u}_{t}\right\|_{L^{2}\left(t^{j}, t^{j+1} ; L^{2}(\Omega)^{2}\right)}^{2} \\
+C h^{4}\|\boldsymbol{u}\|_{L^{\infty}\left(0, T ; H^{2}(\Omega)^{2}\right)}^{2}\|\boldsymbol{u}\|_{L^{2}\left(t^{j}, t^{j+1} ; W^{1,4}(\Omega)^{2}\right)^{2}}^{2}
\end{gathered}
$$

Combining the bounds (4.12), (4.13), (4.14), (4.17) and (4.18) and using the fact that $\boldsymbol{u} \in L^{\infty}\left(0, T ; H^{2}(\Omega)^{2}\right)$, the right-hand side of (4.10) is bounded by

$$
\begin{aligned}
\epsilon \Delta t\left\|\tilde{\boldsymbol{e}}^{j+1}\right\|_{X}^{2}+C\left(h^{2}+\Delta t^{2}\right)\left\|\boldsymbol{u}_{t}\right\|_{L^{2}\left(t^{j}, t^{j+1} ; H^{1}(\Omega)^{2}\right)}^{2}+C h^{2}\|\boldsymbol{u}\|_{L^{2}\left(t^{j}, t^{j+1} ; H^{2}(\Omega)^{2}\right)}^{2} \\
\quad+C h^{2} \Delta t\|\boldsymbol{u}\|_{L^{\infty}\left(0, T ; H^{2}(\Omega)^{2}\right)}^{2}+C \Delta t\left\|\boldsymbol{e}^{j}\right\|_{L^{2}(\Omega)}^{2}\|\boldsymbol{u}\|_{L^{\infty}\left(0, T ; H^{2}(\Omega)^{2}\right)}^{2}+2\left|\int_{t^{j}}^{t^{j+1}} b\left(\tilde{\boldsymbol{e}}^{j+1}, p\right)\right| .
\end{aligned}
$$

The last term is estimated by Lemma 4.2. Then, for an appropriate choice of $\epsilon$ and $\eta$ in Lemma 4.2, we obtain:

$$
\begin{aligned}
&\left\|\boldsymbol{e}^{j+1}\right\|_{L^{2}(\Omega)}^{2}-\left\|\boldsymbol{e}^{j}\right\|_{L^{2}(\Omega)}^{2}+\left\|\tilde{\boldsymbol{e}}^{j+1}-\boldsymbol{e}^{j}\right\|_{L^{2}(\Omega)}^{2}+\frac{1}{2}\left\|\boldsymbol{e}^{j+1}-\tilde{\boldsymbol{e}}^{j+1}\right\|_{L^{2}(\Omega)}^{2} \\
&+\frac{1}{2} K \mu \Delta t\left(\left\|\boldsymbol{e}^{j+1}\right\|_{X}^{2}+\left\|\tilde{\boldsymbol{e}}^{j+1}\right\|_{X}^{2}+\left\|\boldsymbol{e}^{j+1}-\tilde{\boldsymbol{e}}^{j+1}\right\|_{X}^{2}\right)+\Delta t \sum_{E \in \mathcal{E}_{h}} \int_{\partial E_{-}}\left|\left\{\boldsymbol{U}^{j}\right\} \cdot \boldsymbol{n}_{E}\right|\left\|\left[\tilde{\boldsymbol{e}}^{j+1}\right]\right\|^{2} \\
&+\Delta t \sum_{E \in \mathcal{E}_{h}} \int_{\left(\partial E_{-}\right) \cap(\partial \Omega)}\left|\boldsymbol{U}^{j} \cdot \boldsymbol{n}_{E}\right|\left\|\tilde{\boldsymbol{e}}^{j+1}\right\|^{2} \leq C \Delta t\left\|\boldsymbol{e}^{j}\right\|_{L^{2}(\Omega)}^{2}\|\boldsymbol{u}\|_{L^{\infty}\left(0, T ; H^{2}(\Omega)^{2}\right)}^{2}+C\left(h^{2}+\Delta t^{2}\right)\left\|\boldsymbol{u}_{t}\right\|_{L^{2}\left(t^{j}, t^{j+1} ; H^{1}(\Omega)^{2}\right)}^{2} \\
& \quad+C h^{2}\|\boldsymbol{u}\|_{L^{2}\left(t^{j}, t^{j+1} ; H^{2}(\Omega)^{2}\right)}^{2}+C h^{2} \Delta t\|\boldsymbol{u}\|_{L^{\infty}\left(0, T ; H^{2}(\Omega)^{2}\right)}^{2}+\left(C h^{2}+2 \Delta t\right)\|\nabla p\|_{L^{2}\left(t^{j}, t^{j+1}, L^{2}(\Omega)^{2}\right)}^{2} \cdot(4.20)
\end{aligned}
$$

Since $\boldsymbol{U}^{0}=R_{h} \boldsymbol{u}_{0}$, we have $\left\|\boldsymbol{e}^{0}\right\|_{L^{2}(\Omega)} \leq C h\left|\boldsymbol{u}_{0}\right|_{H^{1}(\Omega)}$ and hence applying Gronwall's lemma, we have:

$$
\begin{array}{r}
\max _{j}\left\|\boldsymbol{e}^{j}\right\|_{L^{2}(\Omega)}^{2}+\sum_{j=0}^{N-1}\left\|\tilde{\boldsymbol{e}}^{j+1}-\boldsymbol{e}^{j}\right\|_{L^{2}(\Omega)}^{2}+\frac{1}{2} \sum_{j=0}^{N-1}\left\|\tilde{\boldsymbol{e}}^{j+1}-\boldsymbol{e}^{j+1}\right\|_{L^{2}(\Omega)}^{2}+\sum_{j=0}^{N-1} \Delta t \sum_{E \in \mathcal{E}_{h}} \int_{\partial E_{-}}\left|\left\{\boldsymbol{U}^{j}\right\} \cdot \boldsymbol{n}_{E}\right|\left\|\left[\tilde{\boldsymbol{e}}^{j+1}\right]\right\|^{2} \\
+\sum_{j=0}^{N-1} \Delta t \sum_{E \in \mathcal{E}_{h}} \int_{\left(\partial E_{-}\right) \cap(\partial \Omega)}\left|\boldsymbol{U}^{j} \cdot \boldsymbol{n}_{E}\right|\left\|\tilde{\boldsymbol{e}}^{j+1}\right\|^{2}+\frac{1}{2} K \mu \sum_{j=0}^{N-1} \Delta t\left(\left\|\boldsymbol{e}^{j+1}\right\|_{X}^{2}+\left\|\tilde{\boldsymbol{e}}^{j+1}\right\|_{X}^{2}+\left\|\boldsymbol{e}^{j+1}-\tilde{\boldsymbol{e}}^{j+1}\right\|_{X}^{2}\right) \\
\leq C_{1}\left(h^{2}+\Delta t^{2}+\Delta t\right) e^{C_{2} N \Delta t},
\end{array}
$$

whence (4.7).

The following theorem establishes an error estimate for NIPG. We skip the proof which is a straightforward combination of the proofs of Theorem 4.3 and Proposition 2.3. 
Theorem 4.4. We retain the assumptions of Theorem 4.3, but we replace (1.22) by (2.5). Then there exists a constant $C$, independent of $h$ and $\Delta t$, such that

$$
\begin{aligned}
\max _{j}\left\|\boldsymbol{e}^{j}\right\|_{L^{2}(\Omega)}^{2} & +\sum_{j=0}^{N-1}\left\|\tilde{\boldsymbol{e}}^{j+1}-\boldsymbol{e}^{j}\right\|_{L^{2}(\Omega)}^{2}+\frac{1}{2} \sum_{j=0}^{N-1}\left\|\tilde{\boldsymbol{e}}^{j+1}-\boldsymbol{e}^{j+1}\right\|_{L^{2}(\Omega)}^{2} \\
+ & \sum_{j=0}^{N-1}\left(\Delta t \sum_{E \in \mathcal{E}_{h}} \int_{\partial E_{-}}\left|\left\{\boldsymbol{U}^{j}\right\} \cdot \boldsymbol{n}_{E}\right|\left\|\left[\tilde{\boldsymbol{e}}^{j+1}\right]\right\|^{2}+\Delta t \sum_{E \in \mathcal{E}_{h}} \int_{\left(\partial E_{-}\right) \cap(\partial \Omega)}\left|\boldsymbol{U}^{j} \cdot \boldsymbol{n}_{E}\right|\left\|\tilde{\boldsymbol{e}}^{j+1}\right\|^{2}\right) \\
& +\frac{\mu}{2} \sum_{j=0}^{N-1} \Delta t\left(\frac{1}{2}\left\|\boldsymbol{e}^{j+1}\right\|_{X}^{2}+\left\|\tilde{\boldsymbol{e}}^{j+1}\right\|_{X}^{2}\right) \leq C\left(h^{2}+\Delta t^{2}+\Delta t\right) .
\end{aligned}
$$

These two theorems imply immediately the next result.

Corollary 4.5. Under the assumptions of Theorem 4.3 for SIPG or Theorem 4.4 for NIPG, there exists a constant $C$ independent of $h$ and $\Delta t$ such that

(1)

$$
\max _{j}\left\|\tilde{\boldsymbol{e}}^{j}\right\|_{L^{2}(\Omega)}^{2} \leq C\left(h^{2}+\Delta t^{2}+\Delta t\right)
$$

(2)

$$
\max _{j}\left\|\boldsymbol{e}^{j}\right\|_{X} \leq C, \quad \max _{j}\left\|\tilde{\boldsymbol{e}}^{j}\right\|_{X} \leq C
$$

\section{Further ERror EStimAte FOR VELOCITy AND ESTIMATE FOR PRESSURE}

The following theorem sharpens the results of Theorem 4.3 for SIPG.

Theorem 5.1. Under the assumptions of Theorem 4.3 and if $\boldsymbol{u}_{0} \in H^{3 / 2}(\Omega)^{2}$, there exists a constant $C$ and a constant $\delta>0$ independent of $h$ and $\Delta t$ such that for all $\Delta t \leq \delta$, we have

$$
\max _{j}\left\|\boldsymbol{e}^{j}\right\|_{L^{2}(\Omega)}^{2}+\sum_{j=0}^{N-1}\left\|\boldsymbol{e}^{j+1}-\boldsymbol{e}^{j}\right\|_{L^{2}(\Omega)}^{2}+\frac{\mu K}{2} \sum_{j=0}^{N-1} \Delta t\left\|\boldsymbol{e}^{j+1}\right\|_{X}^{2} \leq C\left(h^{2}+\Delta t^{2}\right)
$$

Proof. Now that we have a first estimate for $\boldsymbol{e}^{j}$ and $\tilde{\boldsymbol{e}}^{j}$, we can sharpen the estimate for $\boldsymbol{e}^{j}$ by eliminating $\tilde{\boldsymbol{e}}^{j+1}$ from the error equation. This is achieved by summing the two equations (1.26) and (1.27) and integrating between $t^{j}$ and $t^{j+1}$ :

$$
\begin{aligned}
\forall \boldsymbol{v}_{h} \in \boldsymbol{X}_{h}, \quad\left(\boldsymbol{U}^{j+1}-\boldsymbol{U}^{j}, \boldsymbol{v}_{h}\right)+\mu & \int_{t^{j}}^{t^{j+1}}\left(a\left(\boldsymbol{U}^{j+1}, \boldsymbol{v}_{h}\right)+J_{0}\left(\boldsymbol{U}^{j+1}, \boldsymbol{v}_{h}\right)\right) \\
& +\int_{t^{j}}^{t^{j+1}} b\left(\boldsymbol{v}_{h}, P^{j+1}\right)+\int_{t^{j}}^{t^{j+1}} c^{\boldsymbol{U}^{j}}\left(\boldsymbol{U}^{j} ; \tilde{\boldsymbol{U}}^{j+1}, \boldsymbol{v}_{h}\right)=\int_{t^{j}}^{t^{j+1}}\left(\check{\boldsymbol{f}}^{j+1}, \boldsymbol{v}_{h}\right) .
\end{aligned}
$$


Inserting $R_{h} \boldsymbol{u}^{j}$ and $R_{h} \boldsymbol{u}^{j+1}$, using (1.28) and (1.29) and choosing the test function $\boldsymbol{v}_{h}$ in $\boldsymbol{V}_{h}$ in order to eliminate the discrete pressure, give:

$$
\begin{array}{r}
\forall \boldsymbol{v}_{h} \in \boldsymbol{V}_{h}, \quad\left(\boldsymbol{e}^{j+1}-\boldsymbol{e}^{j}, \boldsymbol{v}_{h}\right)+\mu \int_{t^{j}}^{t^{j+1}}\left(a\left(\boldsymbol{e}^{j+1}, \boldsymbol{v}_{h}\right)+J_{0}\left(\boldsymbol{e}^{j+1}, \boldsymbol{v}_{h}\right)\right)=\int_{t^{j}}^{t^{j+1}}\left(\boldsymbol{u}_{t}, \boldsymbol{v}_{h}\right)+\left(R_{h} \boldsymbol{u}^{j}-R_{h} \boldsymbol{u}^{j+1}, \boldsymbol{v}_{h}\right) \\
+\mu \int_{t^{j}}^{t^{j+1}}\left(a\left(\boldsymbol{u}-R_{h} \boldsymbol{u}^{j+1}, \boldsymbol{v}_{h}\right)+J_{0}\left(\boldsymbol{u}-R_{h} \boldsymbol{u}^{j+1}, \boldsymbol{v}_{h}\right)\right)+\int_{t^{j}}^{t^{j+1}} c\left(\boldsymbol{u} ; \boldsymbol{u}, \boldsymbol{v}_{h}\right) \\
-\int_{t^{j}}^{t^{j+1}} c^{\boldsymbol{U}^{j}}\left(\boldsymbol{U}^{j} ; \tilde{\boldsymbol{U}}^{j+1}, \boldsymbol{v}_{h}\right)+\int_{t^{j}}^{t^{j+1}} b\left(\boldsymbol{v}_{h}, p-r_{h} p\right) .
\end{array}
$$

Taking $\boldsymbol{v}_{h}=\boldsymbol{e}^{j+1} \in \boldsymbol{V}_{h}$ and applying (1.22), we obtain:

$$
\begin{aligned}
\frac{1}{2}\left(\left\|\boldsymbol{e}^{j+1}\right\|_{L^{2}(\Omega)}^{2}-\left\|\boldsymbol{e}^{j}\right\|_{L^{2}(\Omega)}^{2}+\left\|\boldsymbol{e}^{j+1}-\boldsymbol{e}^{j}\right\|_{L^{2}(\Omega)}^{2}\right)+\mu K \Delta t\left\|\boldsymbol{e}^{j+1}\right\|_{X}^{2} \\
\leq\left|\left(\int_{t^{j}}^{t^{j+1}} \frac{\partial}{\partial t}\left(\boldsymbol{u}-R_{h} \boldsymbol{u}\right), \boldsymbol{e}^{j+1}\right)\right|+\mu\left|\int_{t^{j}}^{t^{j+1}}\left(a\left(\boldsymbol{u}-R_{h} \boldsymbol{u}^{j+1}, \boldsymbol{e}^{j+1}\right)+J_{0}\left(\boldsymbol{u}-R_{h} \boldsymbol{u}^{j+1}, \boldsymbol{e}^{j+1}\right)\right)\right| \\
\quad+\left|\int_{t^{j}}^{t^{j+1}} c\left(\boldsymbol{u} ; \boldsymbol{u}, \boldsymbol{e}^{j+1}\right)-\int_{t^{j}}^{t^{j+1}} c^{\boldsymbol{U}^{j}}\left(\boldsymbol{U}^{j} ; \tilde{\boldsymbol{U}}^{j+1}, \boldsymbol{e}^{j+1}\right)\right|+\left|\int_{t^{j}}^{t^{j+1}} b\left(\boldsymbol{e}^{j+1}, p-r_{h} p\right)\right|
\end{aligned}
$$

The first three linear terms are bounded as in Theorem 4.3. For the pressure term, considering the definition of the approximation operator $r_{h}$, we have

$$
\left|b\left(\boldsymbol{e}^{j+1}, p-r_{h} p\right)\right| \leq C J_{0}\left(\boldsymbol{e}^{j+1}, \boldsymbol{e}^{j+1}\right)^{1 / 2} h|p|_{H^{1}(\Omega)} .
$$

The difficulty is to bound the nonlinear terms; we split them as follows:

$$
\begin{aligned}
c\left(\boldsymbol{u} ; \boldsymbol{u}, \boldsymbol{e}^{j+1}\right)-c^{\boldsymbol{U}^{j}}\left(\boldsymbol{U}^{j} ; \tilde{\boldsymbol{U}}^{j+1}, \boldsymbol{e}^{j+1}\right)=c\left(\boldsymbol{u}-R_{h} \boldsymbol{u}^{j} ; \boldsymbol{u}, \boldsymbol{e}^{j+1}\right)-c\left(\boldsymbol{e}^{j} ; \boldsymbol{u}, \boldsymbol{e}^{j+1}\right) \\
\quad+c^{\boldsymbol{U}^{j}}\left(\boldsymbol{U}^{j} ; \boldsymbol{u}-R_{h} \boldsymbol{u}, \boldsymbol{e}^{j+1}\right)+c^{\boldsymbol{U}^{j}}\left(\boldsymbol{U}^{j} ; R_{h}\left(\boldsymbol{u}-\boldsymbol{u}^{j+1}\right), \boldsymbol{e}^{j+1}\right)-c^{\boldsymbol{U}^{j}}\left(\boldsymbol{U}^{j} ; \tilde{\boldsymbol{e}}^{j+1}, \boldsymbol{e}^{j+1}\right) .
\end{aligned}
$$

First, as in Theorem 4.3 (cf. (4.18)), we have

$\left|c\left(\boldsymbol{u}-R_{h} \boldsymbol{u}^{j} ; \boldsymbol{u}, \boldsymbol{e}^{j+1}\right)\right| \leq C\left\|\boldsymbol{e}^{j+1}\right\|_{X}\left(\|\boldsymbol{u}\|_{L^{\infty}\left(0, T ; W^{1,4}(\Omega)^{2}\right)}\left(t-t^{j}\right)^{1 / 2}\left\|\boldsymbol{u}_{t}\right\|_{L^{2}\left(t^{j}, t^{j+1} ; L^{2}(\Omega)^{2}\right)}+h^{2}\left|\boldsymbol{u}^{j}\right|_{H^{2}(\Omega)}|\boldsymbol{u}|_{W^{1,4}(\Omega)}\right)$.

Using Proposition 4.1, we have, for some $r>2$

$$
\left|c\left(\boldsymbol{e}^{j} ; \boldsymbol{u}, \boldsymbol{e}^{j+1}\right)\right| \leq C|\boldsymbol{u}|_{W^{1, r}(\Omega)}\left\|\boldsymbol{e}^{j+1}\right\|_{X}\left\|\boldsymbol{e}^{j}\right\|_{L^{2}(\Omega)} .
$$

In view of $(1.16)$, we write

$$
{ }_{c}^{\boldsymbol{U}^{j}}\left(\boldsymbol{U}^{j} ; \boldsymbol{u}-R_{h} \boldsymbol{u}, \boldsymbol{e}^{j+1}\right)=-{ }_{\bar{c}} \boldsymbol{U}^{j}\left(\boldsymbol{U}^{j} ; \boldsymbol{e}^{j+1}, \boldsymbol{u}-R_{h} \boldsymbol{u}\right)=-{ }_{\bar{c}} \boldsymbol{U}^{j}\left(\boldsymbol{e}^{j} ; \boldsymbol{e}^{j+1}, \boldsymbol{u}-R_{h} \boldsymbol{u}\right)-\bar{c}^{j}\left(R_{h} \boldsymbol{u}^{j} ; \boldsymbol{e}^{j+1}, \boldsymbol{u}-R_{h} \boldsymbol{u}\right)
$$

For the first term, using the approximation properties of $R_{h}$ and the fact that, according to Corollary $4.5,\left\|\boldsymbol{e}^{j}\right\|_{X}$ is bounded by a constant independent of $j, h$ and $\Delta t$, we obtain

$$
\left|{ }_{\bar{c}} \boldsymbol{U}^{j}\left(\boldsymbol{e}^{j} ; \boldsymbol{e}^{j+1}, \boldsymbol{u}-R_{h} \boldsymbol{u}\right)\right| \leq C h^{3 / 2}|\boldsymbol{u}|_{H^{2}(\Omega)}\left\|\boldsymbol{e}^{j+1}\right\|_{X}
$$


Similarly, the approximation properties of $R_{h}$ imply that, for some $r>2$,

$$
\left|\bar{c} \boldsymbol{U}^{j}\left(R_{h} \boldsymbol{u}^{j} ; \boldsymbol{e}^{j+1}, \boldsymbol{u}-R_{h} \boldsymbol{u}\right)\right| \leq C h^{2}|\boldsymbol{u}|_{H^{2}(\Omega)}\left|\boldsymbol{u}^{j}\right|_{W^{1, r}(\Omega)}\left\|\boldsymbol{e}^{j+1}\right\|_{X} .
$$

Next

$$
{ }_{c} \boldsymbol{U}^{j}\left(\boldsymbol{U}^{j} ; R_{h}\left(\boldsymbol{u}-\boldsymbol{u}^{j+1}\right), \boldsymbol{e}^{j+1}\right)=c^{\boldsymbol{U}^{j}}\left(\boldsymbol{e}^{j} ; R_{h}\left(\boldsymbol{u}-\boldsymbol{u}^{j+1}\right), \boldsymbol{e}^{j+1}\right)+c^{\boldsymbol{U}^{j}}\left(R_{h} \boldsymbol{u}^{j} ; R_{h}\left(\boldsymbol{u}-\boldsymbol{u}^{j+1}\right), \boldsymbol{e}^{j+1}\right) .
$$

For the first term, we use (4.3), (1.36) and Corollary 4.5:

$$
\begin{aligned}
\left|c \boldsymbol{U}^{j}\left(\boldsymbol{e}^{j} ; R_{h}\left(\boldsymbol{u}-\boldsymbol{u}^{j+1}\right), \boldsymbol{e}^{j+1}\right)\right| \leq C & \left\|\boldsymbol{e}^{j}\right\|_{X}\left|\boldsymbol{u}^{j+1}-\boldsymbol{u}\right|_{H^{1}(\Omega)}\left\|\boldsymbol{e}^{j+1}\right\|_{X} \\
& \leq C\left|\boldsymbol{u}^{j+1}-\boldsymbol{u}\right|_{H^{1}(\Omega)}\left\|\boldsymbol{e}^{j+1}\right\|_{X} \leq C\left(t^{j+1}-t\right)^{1 / 2}\left\|\boldsymbol{u}_{t}\right\|_{L^{2}\left(t^{j}, t^{j+1}, H^{1}(\Omega)^{2}\right)}\left\|\boldsymbol{e}^{j+1}\right\|_{X} .
\end{aligned}
$$

The second term is bounded like (4.16). Therefore

$$
\left|c \boldsymbol{U}^{j}\left(\boldsymbol{U}^{j} ; R_{h}\left(\boldsymbol{u}-\boldsymbol{u}^{j+1}\right), \boldsymbol{e}^{j+1}\right)\right| \leq C\left(t^{j+1}-t\right)^{1 / 2}\left\|\boldsymbol{e}^{j+1}\right\| x\left\|\boldsymbol{u}_{t}\right\|_{L^{2}\left(t^{j}, t^{j+1} ; H^{1}(\Omega)^{2}\right)}\|\boldsymbol{u}\|_{L^{\infty}\left(0, T ; H^{1}(\Omega)^{2}\right)} .
$$

Finally, applying (1.16), we write

$$
{ }_{c} \boldsymbol{U}^{j}\left(\boldsymbol{U}^{j} ; \tilde{\boldsymbol{e}}^{j+1}, \boldsymbol{e}^{j+1}\right)=-{ }_{\bar{c}} \boldsymbol{U}^{j}\left(\boldsymbol{U}^{j} ; \boldsymbol{e}^{j+1}, \tilde{\boldsymbol{e}}^{j+1}\right)=-{ }_{\bar{c}} \boldsymbol{U}^{j}\left(\boldsymbol{e}^{j} ; \boldsymbol{e}^{j+1}, \tilde{\boldsymbol{e}}^{j+1}\right)-\bar{c}^{\boldsymbol{U}^{j}}\left(R_{h} \boldsymbol{u}^{j} ; \boldsymbol{e}^{j+1}, \tilde{\boldsymbol{e}}^{j+1}\right) .
$$

For the first term, applying Theorem 3.8, Theorem 4.3, Corollary 4.5 and (1.25)

$$
\begin{array}{r}
\mid \begin{array}{l}
\boldsymbol{U}^{j} \\
\left(\boldsymbol{e}^{j} ; \boldsymbol{e}^{j+1}, \tilde{\boldsymbol{e}}^{j+1}\right) \mid \leq C\left\|\boldsymbol{e}^{j}\right\|_{X}\left\|\boldsymbol{e}^{j+1}\right\|_{X}\left\|\tilde{\boldsymbol{e}}^{j+1}\right\|_{L^{4}(\Omega)} \\
\leq C\left\|\boldsymbol{e}^{j}\right\|_{X}\left\|\boldsymbol{e}^{j+1}\right\|_{X}\left(C_{1}\left\|\tilde{\boldsymbol{e}}^{j+1}\right\|_{L^{2}(\Omega)}^{1 / 2}\left\|\tilde{\boldsymbol{e}}^{j+1}\right\|_{X}^{1 / 2}+C_{2} h^{1 / 4} J_{0}\left(\tilde{\boldsymbol{e}}^{j+1}, \tilde{\boldsymbol{e}}^{j+1}\right)^{1 / 4}\left\|\tilde{\boldsymbol{e}}^{j+1}\right\|_{X}^{1 / 2}+C_{3} h^{1 / 2} J_{0}\left(\tilde{\boldsymbol{e}}^{j+1}, \tilde{\boldsymbol{e}}^{j+1}\right)^{1 / 2}\right) \\
\leq C^{*} \Delta t^{1 / 4}\left\|\boldsymbol{e}^{j}\right\|_{X}\left\|\boldsymbol{e}^{j+1}\right\|_{X} .
\end{array}
\end{array}
$$

For the second term, the approximation properties of $R_{h}$ and Corollary 4.5 imply that

$$
\left|\bar{c} \boldsymbol{U}^{j}\left(R_{h} \boldsymbol{u}^{j} ; \boldsymbol{e}^{j+1}, \tilde{\boldsymbol{e}}^{j+1}\right)\right| \leq C\left\|\boldsymbol{e}^{j+1}\right\|_{X}\left|\boldsymbol{u}^{j}\right|_{W^{1,4}(\Omega)}\left\|\tilde{\boldsymbol{e}}^{j+1}\right\|_{L^{2}(\Omega)} \leq C(\Delta t)^{1 / 2}\|\boldsymbol{u}\|_{L^{\infty}\left(0, T ; W^{1, r}(\Omega)^{2}\right)}\left\|\boldsymbol{e}^{j+1}\right\|_{X}
$$

considering that $\boldsymbol{u} \in L^{\infty}\left(0, T ; W^{1,4}(\Omega)^{2}\right)$. Thus, integrating all these terms over $t^{j}$ and $t^{j+1}$ and summing over $j$, the right-hand side of $(5.2)$ is bounded by

$$
\epsilon \sum_{j=0}^{N-1} \Delta t\left\|\boldsymbol{e}^{j+1}\right\|_{X}^{2}+C\left(h^{2}+\Delta t^{2}\right)+C \sum_{j=0}^{N-1} \Delta t\left\|\boldsymbol{e}^{j}\right\|_{L^{2}(\Omega)}^{2}+C^{*} \Delta t^{1 / 4} \sum_{j=0}^{N} \Delta t\left\|\boldsymbol{e}^{j}\right\|_{X}^{2} .
$$

First, let us choose $\delta$ such that $C^{*} \delta^{1 / 4}=\frac{\mu K}{2}$, i.e.

$$
\delta=\left(\frac{\mu K}{2 C^{*}}\right)^{4}
$$


and note that $C^{*}$ does not depend on $\epsilon$. Next, take $\epsilon=\frac{\mu K}{4}$. Then, (5.2) becomes

$$
\begin{gathered}
\max _{j}\left\|\boldsymbol{e}^{j}\right\|_{L^{2}(\Omega)}^{2}+\sum_{j=0}^{N-1}\left\|\boldsymbol{e}^{j+1}-\boldsymbol{e}^{j}\right\|_{L^{2}(\Omega)}^{2}+\frac{\mu K}{2} \sum_{j=0}^{N-1} \Delta t\left\|\boldsymbol{e}^{j+1}\right\|_{X}^{2} \\
\leq\left\|\boldsymbol{e}^{0}\right\|_{L^{2}(\Omega)}^{2}+C\left(h^{2}+\Delta t^{2}\right)+C \sum_{j=0}^{N-1} \Delta t\left\|\boldsymbol{e}^{j}\right\|_{L^{2}(\Omega)}^{2}+\mu K \Delta t\left\|\boldsymbol{e}^{0}\right\|_{X}^{2} \\
\leq C\left(h^{2}+\Delta t^{2}\right)+C \sum_{j=0}^{N-1} \Delta t\left\|\boldsymbol{e}^{j}\right\|_{L^{2}(\Omega)}^{2},
\end{gathered}
$$

by applying the regularity of $\boldsymbol{u}_{0}$, the approximation properties of $R_{h}$ and (1.25). Then, the result follows from Gronwall's lemma.

Similarly, the next theorem sharpens the result of Theorem 4.4 for NIPG; its proof is almost identical to that of Theorem 5.1, but (5.3) is replaced by

$$
\delta=\left(\frac{\mu}{2 C^{*}}\right)^{4}
$$

Theorem 5.2. Under the assumptions of Theorem 4.4 and if $\boldsymbol{u}_{0} \in H^{3 / 2}(\Omega)^{2}$, there exists a constant $C$ and a constant $\delta>0$, independent of $h$ and $\Delta t$, such that for all $\Delta t \leq \delta$, we have

$$
\max _{j}\left\|\boldsymbol{e}^{j}\right\|_{L^{2}(\Omega)}^{2}+\sum_{j=0}^{N-1}\left\|\boldsymbol{e}^{j+1}-\boldsymbol{e}^{j}\right\|_{L^{2}(\Omega)}^{2}+\frac{\mu}{2} \sum_{j=0}^{N-1} \Delta t\left\|\boldsymbol{e}^{j+1}\right\|_{X}^{2} \leq C\left(h^{2}+\Delta t^{2}\right) .
$$

Remark 5.3. We can improve the estimate for $\left\|\tilde{\boldsymbol{e}}^{j}\right\|_{L^{2}(\Omega)}$ by using a bootstrap argument in Theorems 4.3 and 4.4. Indeed, in the case of SIPG, let the assumptions of Theorem 5.1 hold, and let us revisit the last term of (4.19). Owing to the fact that $\boldsymbol{e}^{j}$ belongs to $\boldsymbol{V}_{h}$, this term can be written (without the factor 2)

$$
\begin{array}{rl}
\left|\int_{t^{j}}^{t^{j+1}} b\left(\tilde{\boldsymbol{e}}^{j+1}, p\right)\right|=\mid \int_{t^{j}}^{t^{j+1}} & b\left(\tilde{\boldsymbol{e}}^{j+1}-\boldsymbol{e}^{j}, p\right)+\int_{t^{j}}^{t^{j+1}} b\left(\boldsymbol{e}^{j}, p-r_{h} p\right) \mid \\
& \leq \int_{t^{j}}^{t^{j+1}}\left|\left(\nabla p, \tilde{\boldsymbol{e}}^{j+1}-\boldsymbol{e}^{j}\right)+\sum_{e \in \Gamma_{h}} \int_{e}\left\{p-r_{h} p\right\}\left[\boldsymbol{e}^{j}\right] \cdot \boldsymbol{n}_{e}\right| \\
\leq \Delta t\|p\|_{L^{\infty}\left(0, T ; H^{1}(\Omega)\right)}\left\|\tilde{\boldsymbol{e}}^{j+1}-\boldsymbol{e}^{j}\right\|_{L^{2}(\Omega)}+C h \Delta t^{1 / 2} J_{0}\left(\boldsymbol{e}^{j}, \boldsymbol{e}^{j}\right)^{1 / 2}\|p\|_{L^{2}\left(t^{j}, t^{j+1} ; H^{1}(\Omega)\right)}
\end{array}
$$

To simplify, denote $\mathcal{C}=\|p\|_{L^{\infty}\left(0, T ; H^{1}(\Omega)\right)}$. Then, either

$$
\left\|\tilde{\boldsymbol{e}}^{j+1}-\boldsymbol{e}^{j}\right\|_{L^{2}(\Omega)} \leq 2 \Delta t \mathcal{C}
$$

or

If (5.6) holds, then

$$
\left\|\tilde{\boldsymbol{e}}^{j+1}-\boldsymbol{e}^{j}\right\|_{L^{2}(\Omega)}>2 \Delta t \mathcal{C} .
$$

$$
\left|\int_{t^{j}}^{t^{j+1}} b\left(\tilde{\boldsymbol{e}}^{j+1}, p\right)\right| \leq \frac{1}{2}\left\|\tilde{\boldsymbol{e}}^{j+1}-\boldsymbol{e}^{j}\right\|_{L^{2}(\Omega)}^{2}+C h \Delta t^{1 / 2} J_{0}\left(\boldsymbol{e}^{j}, \boldsymbol{e}^{j}\right)^{1 / 2}\|p\|_{L^{2}\left(t^{j}, t^{j+1} ; H^{1}(\Omega)\right)}
$$


and the first term of this bound is absorbed by the left-hand side of (4.20). Since all the remaining terms are of the order $\mathcal{O}\left(h^{2}+\Delta t^{2}\right)$, then the end of the argument of Theorem 4.3 implies that $\sum_{j}\left\|\tilde{\boldsymbol{e}}^{j+1}-\boldsymbol{e}^{j+1}\right\|_{L^{2}(\Omega)}^{2}$ is $\mathcal{O}\left(h^{2}+\Delta t^{2}\right)$. Otherwise, if (5.6) does not hold, (5.5) holds and since we know from Theorem 5.1 that $\max _{j}\left\|\boldsymbol{e}^{j}\right\|_{L^{2}(\Omega)}=\mathcal{O}(h+\Delta t)$, this implies that

$$
\max _{j}\left\|\tilde{e}^{j}\right\|_{L^{2}(\Omega)}=\mathcal{O}(h+\Delta t)
$$

Hence, in all cases, (5.7) holds. The proof for NIPG is the same.

Now, we estimate the pressure. The bound we derive below is not optimal, considering the degree of the polynomials used because the argument of Theorem 5.1 does not give a sharper estimate for $\left\|\tilde{e}^{j}\right\|_{X}$. The only result we have comes from Section 4; we only have

$$
\sum_{j=1}^{N} \Delta t\left\|\tilde{e}^{j}\right\|_{X}^{2}=\mathcal{O}(\Delta t)
$$

whereas, an optimal error for the pressure requires

$$
\sum_{j=1}^{N} \Delta t\left\|\tilde{e}^{j}\right\|_{X}^{2}=\mathcal{O}\left(\Delta t^{2}\right)
$$

Indeed, we shall see that the error estimate for the pressure requires an $L^{2}$ in space and time estimate for the discrete derivative of $\boldsymbol{U}^{j}$. More precisely, we need to show that

$$
\sum_{j=0}^{N-1} \Delta t\left\|\frac{\boldsymbol{e}^{j+1}-\boldsymbol{e}^{j}}{\Delta t}\right\|_{L^{2}(\Omega)}^{2}=\mathcal{O}\left(\Delta t^{2}\right) .
$$

But, we cannot prove this because it makes use of (5.9) in the treatment of the nonlinear term. As it is, we only have the following suboptimal estimate, which is an easy consequence of Theorems 5.1 or 5.2:

$$
\sum_{j=0}^{N-1} \Delta t\left\|\frac{\boldsymbol{e}^{j+1}-\boldsymbol{e}^{j}}{\Delta t}\right\|_{L^{2}(\Omega)}^{2}=\mathcal{O}(\Delta t) .
$$

With this, we prove the following bound for the pressure.

Theorem 5.4. Under the assumptions of Theorem 5.1 for SIPG or Theorem 5.2 for NIPG, there exists a constant $C$ independent of $h$ and $\Delta t$ such that for all $\Delta t \leq \delta$ as defined in (5.3) for SIPG or (5.4) for NIPG, we have

$$
\sum_{j=1}^{N} \Delta t\left\|p^{j}-P^{j}\right\|_{L^{2}(\Omega)}^{2} \leq C\left(h^{2}+\Delta t\right) .
$$

Proof. From (1.29) and (5.1), we have an error equation for $p$ :

$$
\begin{aligned}
\int_{t^{j}}^{t^{j+1}} b\left(\boldsymbol{v}_{h}, p-P^{j+1}\right)= & \left(\boldsymbol{U}^{j+1}-\boldsymbol{U}^{j}-\left(\boldsymbol{u}^{j+1}-\boldsymbol{u}^{j}\right), \boldsymbol{v}_{h}\right)+\mu \int_{t^{j}}^{t^{j+1}}\left(a\left(\boldsymbol{U}^{j+1}-\boldsymbol{u}, \boldsymbol{v}_{h}\right)+J_{0}\left(\boldsymbol{U}^{j+1}-\boldsymbol{u}, \boldsymbol{v}_{h}\right)\right) \\
& +\int_{t^{j}}^{t^{j+1}}\left(c^{\boldsymbol{U}^{j}}\left(\boldsymbol{U}^{j} ; \tilde{\boldsymbol{U}}^{j+1}, \boldsymbol{v}_{h}\right)-c\left(\boldsymbol{u} ; \boldsymbol{u}, \boldsymbol{v}_{h}\right)\right), \quad \forall \boldsymbol{v}_{h} \in \boldsymbol{X}_{h} .
\end{aligned}
$$


Inserting $r_{h} p^{j+1}, R_{h} \boldsymbol{u}^{j+1}$ and $R_{h} \boldsymbol{u}^{j}$ and setting $\xi^{j}=P^{j}-r_{h} p^{j}$, this becomes

$$
\begin{array}{r}
-\int_{t^{j}}^{t^{j+1}} b\left(\boldsymbol{v}_{h}, \xi^{j+1}\right)=-\int_{t^{j}}^{t^{j+1}} b\left(\boldsymbol{v}_{h}, p-r_{h} p^{j+1}\right)+\left(\boldsymbol{e}^{j+1}-\boldsymbol{e}^{j}, \boldsymbol{v}_{h}\right)+\left(R_{h} \boldsymbol{u}^{j+1}-R_{h} \boldsymbol{u}^{j}-\left(\boldsymbol{u}^{j+1}-\boldsymbol{u}^{j}\right), \boldsymbol{v}_{h}\right) \\
+\mu \int_{t^{j}}^{t^{j+1}}\left(a\left(\boldsymbol{e}^{j+1}, \boldsymbol{v}_{h}\right)+J_{0}\left(\boldsymbol{e}^{j+1}, \boldsymbol{v}_{h}\right)\right)+\int_{t^{j}}^{t^{j+1}}\left(c^{\boldsymbol{U}^{j}}\left(\boldsymbol{U}^{j} ; \tilde{\boldsymbol{U}}^{j+1}, \boldsymbol{v}_{h}\right)-c\left(\boldsymbol{u} ; \boldsymbol{u}, \boldsymbol{v}_{h}\right)\right) \\
+\mu \int_{t^{j}}^{t^{j+1}}\left(a\left(R_{h} \boldsymbol{u}^{j+1}-\boldsymbol{u}, \boldsymbol{v}_{h}\right)+J_{0}\left(R_{h} \boldsymbol{u}^{j+1}-\boldsymbol{u}, \boldsymbol{v}_{h}\right)\right), \quad \forall \boldsymbol{v}_{h} \in \boldsymbol{X}_{h} .
\end{array}
$$

From the inf-sup condition (1.24), it suffices to estimate the right-hand side in terms of $\left\|\boldsymbol{v}_{h}\right\|_{X}$ for an arbitrary $\boldsymbol{v}_{h} \in \overline{\boldsymbol{X}}_{h}$. This estimate is obtained in much the same way as in the proof of Theorem 5.1 for all terms except the one involving $\boldsymbol{e}^{j+1}-\boldsymbol{e}^{j}$. All the other terms have an optimal upper bound. For $\boldsymbol{e}^{j+1}-\boldsymbol{e}^{j}$, we simply write

$$
\left|\left(\boldsymbol{e}^{j+1}-\boldsymbol{e}^{j}, \boldsymbol{v}_{h}\right)\right| \leq\left\|\boldsymbol{e}^{j+1}-\boldsymbol{e}^{j}\right\|_{L^{2}(\Omega)}\left\|\boldsymbol{v}_{h}\right\|_{L^{2}(\Omega)} \leq C\left\|\boldsymbol{e}^{j+1}-\boldsymbol{e}^{j}\right\|_{L^{2}(\Omega)}\left\|\nabla \boldsymbol{v}_{h}\right\|_{L^{2}(\Omega)},
$$

by (3.5). When summing over $j$, it is clear in view of (5.11) that the contribution to the error of this term is only $\mathcal{O}\left(h^{2}+\Delta t\right)$.

\section{Coupling COntinuous or nOnCOnforming And Discontinuous Methods}

In this section, we present two possible combinations of continuous $\mathbb{P}_{1}$, nonconforming $\mathbb{P}_{1}$ and $\mathbb{P}_{1}$-SIPG or $\mathbb{P}_{1}$-NIPG in this splitting algorithm. From the computational point of view, they are less costly than the scheme presented in Section 1.

To simplify the presentation, we assume that $\Omega$ is a Lipschitz polygon partitioned into two Lipschitz polygonal subdomains $\Omega_{1}$ and $\Omega_{2}$ with interface $\gamma$, that each $\Omega_{i}$ is subdivided by a regular family of triangulation $\mathcal{E}_{h}^{i}$ that match on $\gamma$. In other words, we do not consider hanging nodes.

In the first method that corresponds to the second strategy announced in the introduction, for step 1 , we use continuous $\mathbb{P}_{1}$ elements in $\Omega_{1}$ and SIPG (resp. NIPG) in $\Omega_{2}$ and for step 2, we use SIPG (resp. NIPG) in the whole domain $\Omega$. Thus, setting

$$
\boldsymbol{X}_{h}^{1}=\left\{\boldsymbol{v}_{h} \in \mathcal{C}^{0}\left(\overline{\Omega_{1}}\right): \forall E \in \mathcal{E}_{h}^{1}, \quad \boldsymbol{v}_{h} \in \mathbb{P}_{1}(E)^{2}, \quad \boldsymbol{v}_{h}=\mathbf{0} \quad \text { on } \partial \Omega \cap \partial \Omega_{1}\right\}
$$

defining $\boldsymbol{X}_{h}^{2}$ by

and setting

$$
\boldsymbol{X}_{h}^{2}=\left\{\boldsymbol{v}_{h} \in L^{2}\left(\Omega_{2}\right)^{2}: \quad \forall E \in \mathcal{E}_{h}^{2}, \quad \boldsymbol{v}_{h} \in \mathbb{P}_{1}(E)^{2}\right\}
$$

$$
\tilde{\boldsymbol{X}}_{h}=\left\{\boldsymbol{v}_{h} \in L^{2}(\Omega)^{2}:\left.\quad \boldsymbol{v}_{h}\right|_{\Omega_{1}} \in \boldsymbol{X}_{h}^{1},\left.\quad \boldsymbol{v}_{h}\right|_{\Omega_{2}} \in \boldsymbol{X}_{h}^{2}\right\},
$$

we replace (1.26) by: knowing $\boldsymbol{U}^{j} \in \boldsymbol{V}_{h}$, find $\tilde{\boldsymbol{U}}^{j+1} \in \tilde{\boldsymbol{X}}_{h}$ solution of

$$
\forall \boldsymbol{v}_{h} \in \tilde{\boldsymbol{X}}_{h}, \quad \frac{1}{\Delta t}\left(\tilde{\boldsymbol{U}}^{j+1}-\boldsymbol{U}^{j}, \boldsymbol{v}_{h}\right)+\mu\left(a\left(\tilde{\boldsymbol{U}}^{j+1}, \boldsymbol{v}_{h}\right)+J_{0}\left(\tilde{\boldsymbol{U}}^{j+1}, \boldsymbol{v}_{h}\right)\right)+c^{\boldsymbol{U}^{j}}\left(\boldsymbol{U}^{j} ; \tilde{\boldsymbol{U}}^{j+1}, \boldsymbol{v}_{h}\right)=\left(\check{\boldsymbol{f}}^{j+1}, \boldsymbol{v}_{h}\right),
$$

and keep (1.27) and (1.28) unchanged. Since the forms $a, J_{0}$ and $c$ are consistent, we can denote them by the same symbol in (6.1) although from a computational point of view, they simplify on $\Omega_{1}$; in particular, there are no jump terms and no upwind in $\Omega_{1}$. It is easy to see that the estimates in Section 4 remain valid for this discretization. However the improved estimates of Section 5 do not appear to carry on here, because the spaces $\boldsymbol{X}_{h}$ and $\tilde{\boldsymbol{X}}_{h}$ are different. Thus, this scheme seems less accurate but it requires fewer degrees of freedom. When the spaces $\boldsymbol{X}_{h}$ and $\tilde{\boldsymbol{X}}_{h}$ are different (and more precisely $\tilde{\boldsymbol{X}}_{h}$ is a proper subspace of $\boldsymbol{X}_{h}$ ), then Equations (1.26) and (1.27) can no longer be summed because they are not stated with the same test functions. Indeed, on one hand (1.26) cannot be stated with discontinuous test functions in $\Omega_{1}$. And on the other hand, (1.27) cannot be 
stated with continuous $\mathbb{P}_{1}$ functions, since they do not satisfy the inf-sup condition when combined with $\mathbb{P}_{0}$ pressures. Hence, (5.1), that is the starting point of Theorem 5.1, does not hold.

In the second method that corresponds to the third strategy announced in the introduction, we use the same decomposition of $\Omega$ and the same spaces in both steps. In $\Omega_{1}$, we replace the continuous $\mathbb{P}_{1}$ approximation of $\boldsymbol{u}$ by a $\mathbb{P}_{1}$ nonconforming method. More precisely, let $\Gamma_{h}^{1}$ denote the set of edges of $\mathcal{E}_{h}^{1}$ that do not lie on the interface $\gamma$, let $\Gamma_{h}^{2}=\Gamma_{h} \backslash \Gamma_{h}^{1}$ and define

$$
\overline{\boldsymbol{X}}_{h}^{1}=\left\{\boldsymbol{v}_{h} \in L^{2}\left(\Omega_{1}\right)^{2}: \quad \forall E \in \mathcal{E}_{h}^{1},\left.\quad \boldsymbol{v}_{h}\right|_{E} \in \mathbb{P}_{1}(E)^{2}, \quad \forall e \in \Gamma_{h}^{1}, \quad \int_{e}\left[\boldsymbol{v}_{h}\right]=\mathbf{0}\right\}
$$

which is very similar to (1.23). The space $\boldsymbol{X}_{h}^{2}$ is defined as above and we set

$$
\boldsymbol{X}_{h}=\left\{\boldsymbol{v}_{h} \in L^{2}(\Omega)^{2}:\left.\quad \boldsymbol{v}_{h}\right|_{\Omega_{1}} \in \boldsymbol{X}_{h}^{1},\left.\quad \boldsymbol{v}_{h}\right|_{\Omega_{2}} \in \boldsymbol{X}_{h}^{2}\right\}
$$

It is easy to see that the bilinear form $a$ and the trilinar form $c$ apply to all three SIPG, NIPG and the $\mathbb{P}_{1^{-}}$ nonconforming method. On the other hand, the jump $J_{0}$ is not required although it does not necessarily vanish in the nonconforming method. Thus we replace (1.9) by

$$
J_{0}(\boldsymbol{u}, \boldsymbol{v})=\sum_{e \in \Gamma_{h}^{2}} \frac{\sigma_{e}}{|e|} \int_{e}[\boldsymbol{u}] \cdot[\boldsymbol{v}] .
$$

With this new space $\boldsymbol{X}_{h}$ and new form $J_{0}$, the formulation of this scheme is given again by (1.26), (1.27) and (1.28). As for the first method, the estimates of Section 4 are valid here. In addition, since the same space is used in both steps, the estimates of Section 5 are also valid and therefore, as far as the velocity is concerned, this second method has an optimal accurary. It requires less degrees of freedom than the SIPG or NIPG method and it retains the property of local mass conservation.

\section{Numerical EXPERIMENTS}

Let $\Omega=] 0,1[\times] 0,1[$ and consider the transient Navier-Stokes equations $(0.4)-(0.7)$ with solution

$$
\boldsymbol{u}=\left(\left(x^{4}-2 x^{3}+x^{2}\right)\left(4 y^{3}-6 y^{2}+2 y\right) t,-\left(4 x^{3}-6 x^{2}+2 x\right)\left(y^{4}-2 y^{3}+y^{2}\right) t\right), \quad p=0 .
$$

We study here the numerical convergence of the scheme (1.26)-(1.28) introduced in Section 1, but instead of restricting the discussion to $\mathbb{P}_{1}-\mathbb{P}_{0}$, we also compute the solution with $\mathbb{P}_{2}-\mathbb{P}_{1}$ (that also satisfies the inf-sup condition, see [14]). The time step $\Delta t$ is chosen accordingly so that it is of the order $h$ for the case $\mathbb{P}_{1}-\mathbb{P}_{0}$ and of the order $h^{2}$ for the case $\mathbb{P}_{2}-\mathbb{P}_{1}$. The domain is subdivided into an initial mesh consisting of two elements. We then successively refine the mesh and compute the errors $e_{h}$ on the mesh of size $h$ and the numerical convergence rates by the ratio $\ln \left(e_{h} / e_{h / 2}\right) / \ln (2)$. We present the numerical errors of the velocity in the energy norm and in the $L^{2}$ norm and the numerical error of the pressure in the $L^{2}$ norm computed at the final time of simulation. We choose a constant penalty parameter $\sigma_{e}=10$ for SIPG and we consider three cases for NIPG: $\sigma_{e}=0, \sigma_{e}=1$ and $\sigma_{e}=10$. We did explore the case of SIPG with $\sigma_{e}=1$, but the results were inconclusive. In the following tables, the number after the name SIPG or NIPG corresponds to the value of $\sigma_{e}$.

Table 1 shows the errors and convergence rates for the case where the velocities are approximated by piecewise linears and the pressure by piecewise constants. As predicted by the theory, we observe that the error of $\boldsymbol{u}$ in the $H_{0}^{1}$ norm is $\mathcal{O}(h)$. The first interesting point in this table is that the error of $p$ in the $L^{2}$ norm is $\mathcal{O}(h)$ and that of $\boldsymbol{u}$ is $\mathcal{O}\left(h^{2}\right)$, much better than what the theory predicts. The second interesting point is that the results for NIPG are also optimal, even better in some cases than SIPG, and in this experiment are not sensitive to the choices of $\sigma_{e}$. Let us recall that usually, the advantage of NIPG is that the penalty parameter $\sigma_{e}$ does not have to be adjusted and can be kept small, i.e. $\sigma_{e}=1$. The third interesting point is that NIPG with $\sigma_{e}=0$ (i.e. 
TABLE 1. Numerical errors and convergence rates, for $\mathbb{P}_{1}-\mathbb{P}_{0}$ with $\Delta t=10^{-2}$.

\begin{tabular}{rrrr|rr|rr}
\hline Method & $\mathrm{h}$ & $\left\|\boldsymbol{u}(T)-\boldsymbol{U}^{N}\right\|_{H_{0}^{1}(\Omega)}$ & rate & $\left\|\boldsymbol{u}(T)-\boldsymbol{U}^{N}\right\|_{L^{2}(\Omega)}$ & rate & $\left\|p(T)-P^{N}\right\|_{L^{2}(\Omega)}$ & rate \\
\hline SIPG 10 & $1 / 2$ & $5.113 \times 10^{-3}$ & & $3.748 \times 10^{-4}$ & & $1.432 \times 10^{-3}$ & \\
& $1 / 4$ & $3.123 \times 10^{-3}$ & 0.711 & $1.736 \times 10^{-4}$ & 1.110 & $1.395 \times 10^{-3}$ & 0.038 \\
& $1 / 8$ & $1.651 \times 10^{-3}$ & 0.919 & $6.517 \times 10^{-5}$ & 1.414 & $1.250 \times 10^{-3}$ & 0.159 \\
& $1 / 16$ & $8.208 \times 10^{-4}$ & 1.009 & $2.055 \times 10^{-5}$ & 1.665 & $8.797 \times 10^{-4}$ & 0.506 \\
& $1 / 32$ & $4.045 \times 10^{-4}$ & 1.021 & $5.725 \times 10^{-6}$ & 1.844 & $5.163 \times 10^{-4}$ & 0.769 \\
& $1 / 64$ & $2.006 \times 10^{-4}$ & 1.012 & $1.502 \times 10^{-6}$ & 1.930 & $2.767 \times 10^{-4}$ & 0.899 \\
\hline NIPG 10 & $1 / 2$ & $4.937 \times 10^{-3}$ & & $3.549 \times 10^{-4}$ & & $8.497 \times 10^{-4}$ & \\
& $1 / 4$ & $2.968 \times 10^{-3}$ & 0.734 & $1.326 \times 10^{-4}$ & 1.419 & $1.174 \times 10^{-3}$ & -0.467 \\
& $1 / 8$ & $1.580 \times 10^{-3}$ & 0.909 & $4.091 \times 10^{-5}$ & 1.697 & $1.219 \times 10^{-3}$ & -0.547 \\
& $1 / 16$ & $7.995 \times 10^{-4}$ & 0.983 & $1.151 \times 10^{-5}$ & 1.829 & $8.804 \times 10^{-4}$ & 0.470 \\
& $1 / 32$ & $3.995 \times 10^{-4}$ & 1.001 & $3.037 \times 10^{-6}$ & 1.923 & $5.166 \times 10^{-4}$ & 0.769 \\
& $1 / 64$ & $1.995 \times 10^{-4}$ & 1.002 & $7.781 \times 10^{-7}$ & 1.964 & $2.767 \times 10^{-4}$ & 0.901 \\
\hline NIPG 1 & $1 / 2$ & $5.569 \times 10^{-3}$ & & $6.990 \times 10^{-4}$ & & $5.649 \times 10^{-4}$ & \\
& $1 / 4$ & $3.129 \times 10^{-3}$ & 0.832 & $2.654 \times 10^{-4}$ & 1.397 & $1.255 \times 10^{-3}$ & -1.152 \\
& $1 / 8$ & $1.567 \times 10^{-3}$ & 0.997 & $8.047 \times 10^{-5}$ & 1.721 & $6.290 \times 10^{-4}$ & 0.997 \\
& $1 / 16$ & $7.698 \times 10^{-4}$ & 1.025 & $2.193 \times 10^{-5}$ & 1.875 & $2.491 \times 10^{-4}$ & 1.336 \\
& $1 / 32$ & $3.789 \times 10^{-4}$ & 1.023 & $5.647 \times 10^{-6}$ & 1.957 & $9.425 \times 10^{-5}$ & 1.402 \\
& $1 / 64$ & $1.876 \times 10^{-4}$ & 1.014 & $1.422 \times 10^{-6}$ & 1.989 & $3.777 \times 10^{-5}$ & 1.319 \\
\hline NIPG 0 & $1 / 2$ & $6.396 \times 10^{-3}$ & & $1.041 \times 10^{-3}$ & & $9.736 \times 10^{-4}$ & \\
& $1 / 4$ & $3.940 \times 10^{-3}$ & 0.699 & $4.829 \times 10^{-4}$ & 1.107 & $1.630 \times 10^{-3}$ & -0.743 \\
$1 / 8$ & $2.134 \times 10^{-3}$ & 0.885 & $1.975 \times 10^{-4}$ & 1.290 & $7.075 \times 10^{-4}$ & 1.204 \\
& $1 / 16$ & $1.095 \times 10^{-3}$ & 0.962 & $8.738 \times 10^{-5}$ & 1.177 & $3.013 \times 10^{-4}$ & 1.232 \\
$1 / 32$ & $5.516 \times 10^{-4}$ & 0.989 & $4.178 \times 10^{-5}$ & 1.064 & $1.381 \times 10^{-4}$ & 1.125 \\
$1 / 64$ & $2.763 \times 10^{-4}$ & 0.997 & $2.058 \times 10^{-5}$ & 1.022 & $6.715 \times 10^{-5}$ & 1.040 \\
\hline & & & & & & &
\end{tabular}

without jumps) gives good results, except for the error of $\boldsymbol{u}$ in $L^{2}$. This is surprising because there is no error analysis for NIPG 0 . Since this method is not adapted to the $\mathbb{P}_{1}$ discretization of time independent elliptic problems, this good performance here may be due to the effect of the time derivative.

We repeated the experiments for the case where the velocities are approximated by piecewise quadratics and the pressure by piecewise linears. The results are shown in Table 2. All methods converge optimally in energy norm for velocity and in $L^{2}$ norm for pressure. SIPG 10 is also optimal in $L^{2}$ for the velocity, but the NIPG methods are suboptimal and only of the order $\mathcal{O}\left(h^{2}\right)$. This is consistent with previous results with NIPG for elliptic problems, namely optimal results in the $L^{2}$ norm are only observed when the degree of the polynomial used is odd.

\section{Concluding remarks:}

In this work, we presented several discretizations based on an operator-splitting technique. Besides the advantages of the decoupling of the incompressibility condition and the nonlinearity, our proposed methods benefit from the advantages of the discontinuous Galerkin methods: local mass conservation, high order of 
TABLE 2. Numerical errors and convergence rates, for $\mathbb{P}_{2}-\mathbb{P}_{1}$ with $\Delta t=10^{-3}$.

\begin{tabular}{lrrr|rr|rr}
\hline Method & $\mathrm{h}$ & $\left\|\boldsymbol{u}(T)-\boldsymbol{U}^{N}\right\|_{H_{0}^{1}(\Omega)}$ & rate & $\left\|\boldsymbol{u}(T)-\boldsymbol{U}^{N}\right\|_{L^{2}(\Omega)}$ & rate & $\left\|p(T)-P^{N}\right\|_{L^{2}(\Omega)}$ & rate \\
\hline SIPG 10 & $1 / 2$ & $2.920 \times 10^{-4}$ & & $1.265 \times 10^{-5}$ & & $8.253 \times 10^{-5}$ & \\
& $1 / 4$ & $8.922 \times 10^{-5}$ & 1.711 & $1.948 \times 10^{-6}$ & 2.699 & $2.745 \times 10^{-5}$ & 1.588 \\
& $1 / 8$ & $2.309 \times 10^{-5}$ & 1.950 & $2.522 \times 10^{-7}$ & 2.950 & $7.741 \times 10^{-6}$ & 1.826 \\
& $1 / 16$ & $5.693 \times 10^{-6}$ & 2.020 & $3.104 \times 10^{-8}$ & 3.022 & $2.150 \times 10^{-6}$ & 1.848 \\
& $1 / 32$ & $1.399 \times 10^{-6}$ & 2.025 & $3.813 \times 10^{-9}$ & 3.025 & $5.756 \times 10^{-7}$ & 1.901 \\
\hline NIPG 10 & $1 / 2$ & $2.519 \times 10^{-4}$ & & $1.233 \times 10^{-5}$ & & $6.761 \times 10^{-5}$ & \\
& $1 / 4$ & $7.355 \times 10^{-5}$ & 1.776 & $1.823 \times 10^{-6}$ & 2.758 & $3.896 \times 10^{-5}$ & 0.795 \\
& $1 / 8$ & $1.953 \times 10^{-5}$ & 1.913 & $2.554 \times 10^{-7}$ & 2.835 & $8.512 \times 10^{-6}$ & 2.194 \\
& $1 / 16$ & $4.940 \times 10^{-6}$ & 1.983 & $3.917 \times 10^{-8}$ & 2.705 & $1.869 \times 10^{-6}$ & 2.186 \\
& $1 / 32$ & $1.236 \times 10^{-6}$ & 2.00 & $7.563 \times 10^{-9}$ & 2.373 & $4.399 \times 10^{-7}$ & 2.088 \\
\hline NIPG 1 & $1 / 2$ & $2.654 \times 10^{-4}$ & & $1.407 \times 10^{-5}$ & & $2.274 \times 10^{-4}$ & \\
& $1 / 4$ & $7.941 \times 10^{-5}$ & 1.741 & $2.398 \times 10^{-6}$ & 2.553 & $5.230 \times 10^{-5}$ & 2.121 \\
& $1 / 8$ & $2.117 \times 10^{-5}$ & 1.907 & $3.646 \times 10^{-7}$ & 2.717 & $1.130 \times 10^{-5}$ & 2.210 \\
& $1 / 16$ & $5.340 \times 10^{-6}$ & 1.987 & $6.086 \times 10^{-8}$ & 2.583 & $2.378 \times 10^{-6}$ & 2.249 \\
& $1 / 32$ & $1.331 \times 10^{-6}$ & 2.003 & $1.261 \times 10^{-8}$ & 2.270 & $5.189 \times 10^{-7}$ & 2.197 \\
\hline NIPG 0 & $1 / 2$ & $2.712 \times 10^{-4}$ & & $1.513 \times 10^{-5}$ & & $2.567 \times 10^{-4}$ & \\
& $1 / 4$ & $8.473 \times 10^{-5}$ & 1.678 & $2.783 \times 10^{-6}$ & 2.443 & $6.396 \times 10^{-5}$ & 2.005 \\
& $1 / 8$ & $2.287 \times 10^{-5}$ & 1.889 & $4.314 \times 10^{-7}$ & 2.690 & $1.425 \times 10^{-5}$ & 2.165 \\
& $1 / 16$ & $5.766 \times 10^{-6}$ & 1.988 & $7.344 \times 10^{-8}$ & 2.554 & $3.033 \times 10^{-6}$ & 2.233 \\
& $1 / 32$ & $1.434 \times 10^{-6}$ & 2.008 & $1.546 \times 10^{-8}$ & 2.247 & $6.703 \times 10^{-7}$ & 2.178 \\
\hline
\end{tabular}

approximation, robustness and stability. It is to be noted that the SIPG version might be preferred to the NIPG from a point of view of better conditioning of the linear system. Finally our multi-numerics approach (coupling of continuous and discontinuous finite elements) allows the use of efficient solvers for the first step, while still obtaining a locally divergence-free velocity.

\section{REFERENCES}

[1] R.A. Adams, Sobolev Spaces. Academic Press, New York (1975).

[2] A.S. Almgren, J.B. Bell, P. Colella, L.H. Howell and M.L. Welcome, A conservative adaptive projection method for the variable density incompressible Navier-Stokes equations. Technical Report LNBL-39075, UC-405 (1996).

[3] C.E. Baumann and J.T. Oden, A discontinuous hp finite element method for convection-diffusion problems. Comput. Methods Appl. Mech. Engrg. 175 (1999) 311-341.

[4] J. Blasco and R. Codina, Error estimates for an operator-splitting method for incompressible flows. Appl. Numer. Math. 51 (2004) 1-17.

[5] J. Blasco, R. Codina and A. Huerta, A fractional-step method for the incompressible Navier-Stokes equations related to a predictor-multicorrector algorithm. Int. J. Numer. Meth. Fl. 28 (1997) 1391-1419.

[6] P.G. Ciarlet, The finite element methods for elliptic problems. North-Holland, Amsterdam (1978).

[7] A.J. Chorin, Numerical solution of the Navier-Stokes equations. Math. Comp. 22 (1968) 745-762.

[8] M. Crouzeix and P.A. Raviart, Conforming and non conforming finite element methods for solving the stationary Stokes equations. RAIRO Anal. Numér. R3 (1973) 33-76.

[9] C. Dawson and J .Proft, Discontinuous and coupled continuous/discontinuous Galerkin methods for the shallow water equations. Comput. Methods Appl. Mech. Engrg. 191 (2002) 4721-4746. 
[10] C. Dawson, S. Sun and M. Wheeler, Compatible algorithms for coupled flow and transport. Comput. Methods Appl. Mech. Engrg. (2003) 2565-2580.

[11] E. Fernandez-Cara and M.M. Beltram, The convergence of two numerical schemes for the Navier-Stokes equations. Numer. Math. 55 (1989) 33-60.

[12] V. Girault and J.-L. Lions, Two-grid finite-element schemes for the steady Navier-Stokes problem in polyhedra. Portugal. Math. 58 (2001) 25-57.

[13] V. Girault and P.A. Raviart, Finite element methods for Navier-Stokes equations. Lecture Notes in Math. 749, Springer-Verlag, Berlin, Heidelberg, New-York (1979).

[14] V. Girault, B. Rivière and M.F. Wheeler, A discontinuous Galerkin method with non-overlapping domain decomposition for the Stokes and Navier-Stokes problems. Math. Comp. 74 (2005) 53-84.

[15] R. Glowinski, Finite element methods for Incompressible Viscous Flows, in Numerical Methods for Fluids (Part 3), Handbook of Numerical Analysis, 9, Elsevier, North-Holland (2003).

[16] P. Grisvard, Elliptic problems in nonsmooth domains, Pitman Monogr. Studies Pure Appl. Math. 24, Pitman, Boston, MA (1985).

[17] J.L. Guermond and L. Quartapelle, On the approximation of the unsteady Navier-Stokes equations by finite element projection methods. Numer. Math. 80 (1998) 207-238.

[18] J.L. Guermond and J. Shen, Velocity-correction projection methods for incompressible flows. SIAM J. Numer. Anal. 41 (2003) $112-134$.

[19] J.L. Guermond and J. Shen, A new class of truly consistent splitting schemes for incompressible flows. J. Comput. Phys. 192 (2003) 262-276.

[20] S. Kaya and B. Rivière, A discontinuous subgrid eddy viscosity method for the time-dependent Navier-Stokes equations. SIAM J. Numer. Anal. (2005), to appear.

[21] P. Lesaint and P.A. Raviart, On a finite element method for solving the neutron transport equation, in Mathematical Aspects of Finite Element Methods in Partial Differential Equations, C.A. de Boor Ed., Academic Press (1974) 89-123.

[22] J.-L. Lions and E. Magenes, Problèmes aux Limites non Homogènes et Applications, I. Dunod, Paris (1968).

[23] J.-L. Lions, Quelques méthodes de résolution des problèmes aux limites non linéaires. Dunod, Paris (1969).

[24] A. Quarteroni, F. Saleri and A. Veneziani, Factorization methods for the numerical approximation of Navier-Stokes equations. Comput. Methods Appl. Mech. Engrg. 188 (2000) 505-526.

[25] R. Rannacher, On Chorin's projection method for the incompressible Navier-Stokes equations, Navier-Stokes equations: Theory and Numerical Methods, R. Rautmann et al. Eds., Springer (1992).

[26] B. Rivière, M.F. Wheeler and V. Girault, Improved energy estimates for interior penalty, constrained and discontinuous Galerkin methods for elliptic problems. Part I. Comput. Geosci. 3 (1999) 337-360.

[27] R. Temam, Sur l'approximation de la solution des equations de Navier-Stokes par la méthode des pas fractionnaires (I), (II). Arch. Rational Mech. Anal. 33 (1969) 377-385.

[28] R. Temam, Navier-Stokes equations. Theory and numerical analysis. North-Holland, Amsterdam (1979).

[29] S. Turek, On discrete projection methods for the incompressible Navier-Stokes equations: an algorithmic approach. Comput. Methods Appl. Mech. Engrg. 143 (1997) 271-288.

[30] M.F. Wheeler, An elliptic collocation-finite element method with interior penalties. SIAM J. Numer. Anal. 15 (1978) $152-161$.

[31] N.N. Yanenko, The method of fractional steps. The solution of problems of mathematical physics in several variables. SpringerVerlag, New York (1971). 